\title{
Division algebras and
}

\section{supersymmetry III}

\author{
John Huerta \\ CAMGSD, Instituto Superior Técnico, Technical University of Lisbon, \\ Portugal \\ jhuerta@math.ist.utl.pt
}

\begin{abstract}
Recent work applying higher gauge theory to the superstring has indicated the presence of "higher symmetry". Infinitesimally, this is realized by a "Lie 2-superalgebra" extending the Poincaré superalgebra in precisely the dimensions where the classical supersymmetric string makes sense: $3,4,6$ and 10. In the previous paper in this series, we constructed this Lie 2-superalgebra using the normed division algebras. In this paper, we use an elegant geometric technique to integrate this Lie 2-superalgebra to a "Lie 2-supergroup" extending the Poincaré supergroup in the same dimensions.

Briefly, a "Lie 2-superalgebra" is a two-term chain complex with a bracket like a Lie superalgebra, but satisfying the Jacobi identity only up to chain homotopy. Simple examples of Lie 2-superalgebras arise from 3 -cocycles on Lie superalgebras, and it is in this way that we constructed the Lie 2-superalgebra above. Because this 3-cocycle is supported on a nilpotent subalgebra, our geometric technique applies, and we obtain a Lie 2-supergroup integrating the Lie 2-superalgebra in the guise of a smooth 3-cocycle on the Poincaré supergroup.
\end{abstract}

e-print archive: http://lanl.arXiv.org/abs/1109.3574 


\section{Introduction}

There is a deep connection between supersymmetry and the normed division algebras: the real numbers, $\mathbb{R}$, the complex numbers, $\mathbb{C}$, the quaternions, $\mathbb{H}$, and the octonions, $\mathbb{O}$. This is visible in super-Yang-Mills theory and in the classical supersymmetric string and 2-brane. Most simply, it is seen in the dimensions for which these theories make sense. The normed division algebras have dimension $n=1,2,4$ and 8 , while the classical supersymmetric string and super-Yang-Mills make sense in spacetimes of dimension two higher than these: $n+2=3,4,6$ and 10. Similarly, the classical supersymmetric 2-brane makes sense in dimensions three higher: $n+3=4,5,7$ and 11. Intriguingly, when we take quantum mechanics into account, it is only in the octonionic dimensions that the supersymmetric string and 2-brane appear to have a consistent quantization -10 for the superstring, and 11 for M-theory, which incorporates the 2-brane. In this paper, however, our concern is entirely with the classical supersymmetric string: we study algebraic and geometric ingredients used in the classical Green-Schwarz action for the superstring. A parallel story for the supersymmetric 2-brane will be told in a forthcoming paper.

This is the third in a series of papers exploring the relationship between supersymmetry and division algebras $[5,6]$, the first two of which were coauthored with John Baez. In the first paper [5], we reviewed the known story of how the division algebras give rise to the supersymmetry of super-YangMills theory. In the second [6], we showed how the division algebras can be used to construct "Lie 2 -superalgebras" $\mathfrak{s u p e r s t r i n g}(n+1,1)$, which extend the Poincaré superalgebra in the superstring dimensions $n+2=3,4,6$ and 10. In this paper, we will describe a geometric method to integrate these Lie 2-superalgebras to "2-supergroups".

Roughly, a "2-group" is a mathematical gadget like a group, but where the group axioms, such as the associative law:

$$
(g h) k=g(h k)
$$

no longer hold. Instead, they are replaced by isomorphisms:

$$
(g h) k \cong g(h k)
$$

which must satisfy axioms of their own. A "Lie 2-group" is a smooth version of a 2-group, where every set is actually a smooth manifold and every operation is smooth, and a "2-supergroup" is analogous, but replaces manifolds with "supermanifolds". All these concepts (Lie 2-group, supermanifold, and 2-supergroup) will be defined precisely later on. We assume no familiarity with either 2-groups or supergeometry. 
Our motives for constructing these 2-supergroups come from both mathematics and physics. Physically, as we show in our previous paper [6], the existence of the superstring in spacetimes of dimension $n+2=3,4$, 6 and 10 secretly gives rise to a way to extend the Poincare superalgebra, $\mathfrak{s i s o}(n+1,1)$, to a Lie 2 -superalgebra we like to call $\mathfrak{s u p e r s t r i n g}(n+1,1)$. Here, the Poincaré superalgebra is a Lie superalgebra whose even part consists of the infinitesimal rotations $\mathfrak{s o}(n+1,1)$ and translations $V$ on Minkowski spacetime, and whose odd part consists of "supertranslations" $S$, or spinors:

$$
\mathfrak{s i s o}(n+1,1)=\mathfrak{s o}(n+1,1) \ltimes(V \oplus S) .
$$

Naturally, we can identify the translations $V$ with the vectors in Minkowski spacetime, so $V$ carries a Minkowski inner product $g$ with signature $(n+1,1)$. We extend $\mathfrak{s i s o}(n+1,1)$ to a Lie 2 -superalgebra $\mathfrak{s u p e r s t r i n g}(n+1,1)$ defined on the 2-term chain complex:

$$
\mathfrak{s i s o}(n+1,1) \stackrel{d}{\longleftarrow} \mathbb{R}
$$

equipped with some extra structure.

What is the superstring Lie 2-algebra good for? The answer lies in a feature of string theory called the "Kalb-Ramond field", or " $B$ field". The $B$ field couples to strings just as the $A$ field in electromagnetism couples to charged particles. The $A$ field is described locally by a 1 -form, so we can integrate it over a particle's worldline to get the interaction term in the Lagrangian for a charged particle. Similarly, the $B$ field is described locally by a 2 -form, which we can integrate over the worldsheet of a string.

Gauge theory has taught us that the $A$ field has a beautiful geometric meaning: it is a connection on a $\mathrm{U}(1)$ bundle over spacetime. What is the corresponding meaning of the $B$ field? It can be seen as a connection on a "U(1) gerbe": a gadget like a U(1) bundle, but suitable for describing strings instead of point particles. Locally, connections on $U(1)$ gerbes can be identified with 2-forms. But globally, they cannot. The idea that the $B$ field is a $\mathrm{U}(1)$ gerbe connection is implicit in work going back at least to the 1986 paper by Gawedzki [27]. More recently, Freed and Witten [25] showed that the subtle difference between 2 -forms and connections on $\mathrm{U}(1)$ gerbes is actually crucial for understanding anomaly cancellation. In fact, these authors used the language of "Deligne cohomology" rather than gerbes. Later work made the role of gerbes explicit: see for example Carey et al. [15], and also Gawedzki and Reis [26]. 
More recently still, work on higher gauge theory has revealed that the $B$ field can be viewed as part of a larger package. Just as gauge theory uses Lie groups, Lie algebras, and connections on bundles to describe the parallel transport of point particles, higher gauge theory generalizes all these concepts to describe parallel transport of extended objects such as strings and membranes [7,9]. In particular, Sati et al. [45] have developed a theory of " $n$-connections" suitable for describing parallel transport of objects with $n$-dimensional worldvolumes. In their theory, the Lie algebra of the gauge group is replaced by a Lie $n$-algebra - or in the supersymmetric context, a Lie $n$-superalgebra. Applying their ideas to $\mathfrak{s u p e r s t r i n g}(n+1,1)$, we get a 2-connection which can be described locally using the following fields:

\begin{tabular}{cc}
\hline $\mathfrak{s u p e r s t r i n g}(n+1,1)$ & Connection component \\
\hline $\mathbb{R}$ & $\mathbb{R}$-valued 2-form \\
$\downarrow$ & \\
$\mathfrak{s i s o}(n+1,1)$ & $\mathfrak{s i s o}(n+1,1)$-valued 1-form \\
\hline
\end{tabular}

The $\mathfrak{s i s o}(n+1,1)$-valued 1-form consists of three fields which help define the background geometry on which a superstring propagates: the Levi-Civita connection $A$, the vielbein $e$, and the gravitino $\psi$. But the $\mathbb{R}$-valued 2 -form is equally important in the description of this background geometry: it is the $B$ field!

How does $\mathfrak{s u p e r s t r i n g}(n+1,1)$ come to be, and why in only these curious dimensions? The answer concerns the cohomology of the Poincaré superalgebra, $\mathfrak{s i s o}(n+1,1)$. The cohomology theory of Lie algebras, known as Chevalley-Eilenberg cohomology $[1,17]$, can be generalized in a straightforward way to Lie superalgebras [38]. As we describe in our previous paper [6], there is a 3 -cocycle on $\mathfrak{s i s o}(n+1,1)=\mathfrak{s o}(n+1,1) \ltimes(V \oplus S)$ that vanishes unless is eats a vector $v \in V$ and two spinors $\psi, \phi \in S$ :

$$
\alpha(v, \psi, \phi)=g(v, \psi \cdot \phi) .
$$

Here, $g$ denotes the Minkowski inner product on vectors, and $\psi \cdot \phi$ denotes an operation that takes two spinors and gives a vector. It is the spinorspinor part of the bracket on $\mathfrak{s i s o}(n+1,1)$.

One can define $\alpha$ in any dimension. In fact, fixing bases for $V$ and $S$, the components of $\alpha$ are well-known to any physicist: they are the entries of the gamma matrices! However, $\alpha$ is a 3-cocycle in Lie superalgebra cohomology precisely when the dimension of spacetime is $3,4,6$ or 10 . Moreover, this fact is exactly what is required to define the classical supersymmetric string in those dimensions - technically, it is needed for the Green-Schwarz Lagrangian to have "Siegel symmetry", which forces the number of bosonic 
and fermionic degrees of freedom to match [29]. This algebraic story has a beautiful interpretation in terms of division algebras [5, 6, 23, 24, 35].

Just as 2-cocycles on a Lie superalgebra describe central extensions to larger Lie superalgebras, $(n+1)$-cocycles give extensions to Lie $n$-superalgebras. To understand this, we need to know a bit about $L_{\infty}$-algebras $[39,46]$. An $L_{\infty}$-algebra is a chain complex equipped with a structure like that of a Lie algebra, but where the laws hold only "up to $d$ of something". A Lie $n$-algebra is an $L_{\infty}$-algebra in which only the first $n$ terms are nonzero. All these ideas also have "super" versions.

In general, an $\mathfrak{h}$-valued $(n+1)$-cocycle $\omega$ on $\mathfrak{g}$ is a linear map:

$$
\Lambda^{n+1} \mathfrak{g} \rightarrow \mathfrak{h}
$$

satisfying a certain equation called a "cocycle condition". We can use an $\mathfrak{h}$-valued $(n+1)$-cocycle $\omega$ on a Lie superalgebra $\mathfrak{g}$ to extend $\mathfrak{g}$ to a Lie $n$-superalgebra of the following form:

$$
\mathfrak{g} \stackrel{d}{\longleftarrow} 0 \stackrel{d}{\longleftarrow} \cdots \stackrel{d}{\longleftarrow} 0 \stackrel{d}{\longleftarrow} \mathfrak{h} .
$$

Here, $\mathfrak{g}$ sits in degree 0 while $\mathfrak{h}$ sits in degree $n-1$. We call Lie $n$-superalgebras of this form "slim Lie $n$-superalgebras", and denote them by $\mathfrak{b r a n e} \mathfrak{e}_{\omega}(\mathfrak{g}, \mathfrak{h})$. In particular, we can use the 3 -cocycle $\alpha$ to extend $\mathfrak{s i s o}(n+$ $1,1)$ to a slim Lie 2 -superalgebra of the following form:

$$
\mathfrak{s i s o}(n+1,1) \stackrel{d}{\longleftarrow} \mathbb{R} .
$$

This is how we obtain the Lie 2-superalgebra $\mathfrak{s u p e r} \mathfrak{s t r i n g}(n+1,1)$.

We have already mentioned how the 3-cocycle $\alpha$ is required for the classical Green-Schwarz superstring to have Siegel symmetry in dimensions 3, 4, 6 and 10. Of course, it is only in dimension 10 that one expects the superstring to have a consistent quantization. This is because in this dimension, and no others, quantum anomalies cancel. The work of Urs Schreiber et al. shows that $\alpha$ plays a role in this story too, at least for the heterotic string $[45,12]$.

So far, we have focused on Lie 2-algebras and generalized connections valued in them. This connection data is infinitesimal: it tells us how to parallel transport strings a little bit. Ultimately, we would like to understand this parallel transport globally, as we do with particles in ordinary gauge theory.

To achieve this global description, we will need "Lie $n$-groups" rather than Lie $n$-algebras. Naively, one expects a Lie 2 -supergroup $\operatorname{Superstring}(n+1,1)$ 
for which the Lie 2-superalgebra $\mathfrak{s u p e r s t r i n g}(n+1,1)$ is the infinitesimal approximation. In fact, this is precisely what we will construct.

In order to "integrate" Lie $n$-algebras to obtain Lie $n$-groups, we will have to overcome two obstacles: how does one define a Lie $n$-group? And, how does one integrate a Lie $n$-algebra to a Lie $n$-group? To answer the former question, at least for $n=2$, we use Baez and Lauda's definition of Lie 2group: it is a categorified Lie group, a "weak 2-category" with one object with a manifold of weakly associative and weakly invertible morphisms, a manifold of strictly associative and strictly invertible 2-morphisms, and all structure maps smooth. While this definition is known to fall short in important ways, it has the virtue of being fairly simple. Ultimately, one should use an alternative definition, like that of Henriques [31] or Schommer-Pries [47], which weakens the notion of product on a group: rather than an algebraic operation in which there is a unique product of any two group elements, "the" product is defined only up to equivalence.

So, roughly speaking, a Lie $n$-group should be a "weak $n$-category" with one object, a manifold of weakly invertible morphisms, a manifold of weakly invertible 2-morphisms, and so on, up to a manifold of strictly invertible $n$-morphisms. To make this precise, however, we need to get very precise about what a "weak $n$-category" is, which becomes more complicated as $n$ gets larger. We therefore limit ourselves to the tractable case of $n=2$, and further limit ourselves to what we call a "slim Lie 2-group".

A "slim Lie 2-group" is what Baez and Lauda call a "special Lie 2-group": it is a skeletal bicategory with one object, a Lie group $G$ of morphisms, a Lie group $G \ltimes H$ of 2-morphisms, and the group axioms hold strictly except for associativity - there is a nontrivial 2-morphism called the "associator":

$$
a\left(g_{1}, g_{2}, g_{3}\right):\left(g_{1} g_{2}\right) g_{3} \Rightarrow g_{1}\left(g_{2} g_{3}\right) \text {. }
$$

The associator, in turn, satisfies the "pentagon identity", which says the following pentagon commutes:

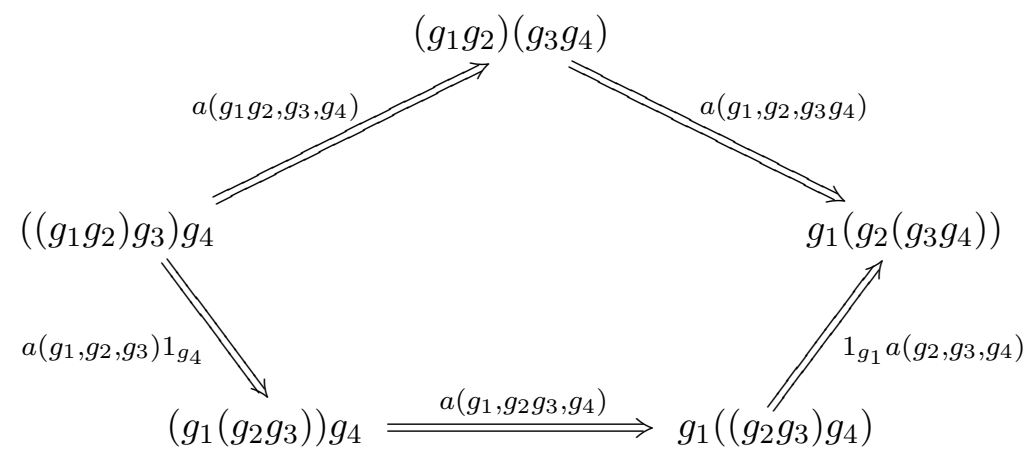


We shall see that this identity forces $a$ to be a 3-cocycle on the Lie group $G$ of morphisms. We denote the Lie 2-group of this from by $\operatorname{String}_{a}(G, H)$.

Moreover, we can generalize all of this to obtain Lie 2-supergroups from 3 -cocycles on Lie supergroups. In general, we expect that any supergroup $(n+1)$-cocycle $f$ gives rise to a slim $n$-supergroup, Brane $f(G, H)$, though this cannot be made precise without being more definite about $n$-categories for higher $n$.

Nonetheless, the precise examples of Lie 2-groups suggest a strong parallel to the way Lie algebra $(n+1)$-cocycles give rise to Lie $n$-algebras. And this parallel suggests a naive scheme to integrate Lie $n$-algebras. Given a slim Lie $n$-superalgebra $\mathfrak{b r a n e}_{\omega}(\mathfrak{g}, \mathfrak{h})$, we seek a slim Lie $n$-supergroup $\operatorname{Brane}_{f}(G, H)$ where:

- $G$ is a Lie supergroup with Lie superalgebra $\mathfrak{g}$; i.e., it is a Lie supergroup integrating $\mathfrak{g}$

- $H$ is an abelian Lie supergroup with Lie superalgebra $\mathfrak{h}$; i.e., it is a Lie supergroup integrating $\mathfrak{h}$,

- $f$ is a Lie supergroup $(n+1)$-cocycle on $G$ that, in some suitable sense, integrates the Lie superalgebra $(n+1)$-cocycle $\omega$ on $\mathfrak{g}$.

Admittedly, we only define $\operatorname{Brane}_{f}(G, H)$ precisely when $n=2$, but that will suffice to handle our example of interest, $\mathfrak{s u p e r s t r i n g}(n+1,1)$.

Unfortunately, this naive scheme fails to work even for well-known examples of slim Lie 2-algebras, such as the string Lie 2-algebra $\mathfrak{s t r i n g}(n)$, whose definition we recall in Section 3.1.1. In this case, we can:

- integrate $\mathfrak{s o}(n)$ to $\operatorname{Spin}(n)$ or $\mathrm{SO}(n)$,

- integrate $\mathbb{R}$ to $\mathbb{R}$ or $\mathrm{U}(1)$,

- but there is no hope to integrate $\omega$ to a nontrivial $(n+1)$-cocycle $f$ on $\mathrm{SO}(n)$ or $\operatorname{Spin}(n)$, because compact Lie groups admit no nontrivial smooth cocycles $[8,55]$.

Really, this failure is a symptom of the fact that our definition of Lie $n$-group is oversimplified. There are more sophisticated approaches to integrating the string Lie 2-algbera, like those due to Baez et al. [10] or SchommerPries [47], and a general technique to integrate any Lie $n$-algebra due to Henriques [31], which Schreiber [50] has in turn generalized to handle Lie $n$-superalgebras and more. All three techniques involve generalizing the notion of Lie 2-group (or Lie $n$-group, for Henriques and Schreiber) away from the world of finite-dimensional manifolds, and the latter three generalize 
the notion of 2-group to one in which products are defined only up to equivalence.

Given this history, it is remarkable that the naive scheme we outlined for integration actually works for the Lie $n$-superalgebra we really care about - namely, the superstring Lie 2-algbera. Moreover, this is not some weird quirk unique to this special case, but the result of a beautiful geometric procedure for integrating Lie algebra cocycles defined on a nilpotent Lie algebra. Originally invented by Houard [32], we generalize this technique to the case of nilpotent Lie superalgebras and supergroups.

This paper is organized as follows. In Section 2, we sketch how to construct a Lie $n$-group from an $(n+1)$-cocycle in smooth group cohomology, and make this precise for $n=2$. In Section 3, we define Lie $n$-superalgebras as $n$-term $L_{\infty}$-superalgebras, and show to construct a Lie $n$-superalgebra from an $(n+1)$-cocycle in Lie superalgebra cohomology. We then give some examples of Lie 2-superalgebras obtained via 3-cocycles: the string Lie 2-algebra in Section 3.1.1, a new example we call the Heisenberg Lie 2-algebra in Section 3.1.2, and the supertranslation Lie 2-superalgebra in Section 3.1.3. Finally, in Section 3.1.4, we give our key example, the superstring Lie 2-superalgebra, $\mathfrak{s u p e r s t r i n g}(n+1,1)$.

We spend the rest of the paper building the machinery to integrate $\mathfrak{s u p e r s t r i n g}(n+1,1)$. In Section 4 , we give some background on the problem of integrating Lie $n$-algebras, and introduce a key construction in Section 4.1: a geometric technique, due to Houard [32], to fill out $p$-simplices in Lie groups given a $(p+1)$-tuple of vertices, provided the Lie group in question is "exponential": the exponential map is a diffeomorphism. This immediately allows us to integrate Lie algebra 3-cocycles to Lie group 3-cocycles for all nilpotent Lie algebras and their simply-connected Lie groups. In Section 4.2, we apply this technique to obtain the Heisenberg Lie 2-group from the Heisenberg Lie 2-algebra. Then we lay the groundwork to generalize this construction to Lie 2-superalgebras and 2-supergroups. In Section 5, we give a brief introduction to supermanifold theory using the "functor of points" approach we learned from Sachse [44] and Balduzzi et al. [11]. In Section 6 , we generalize the results of Section 2 to the super case by showing how to construct a 2 -supergroup from a 3 -cocycle in supergroup cohomology. In Section 7, we generalize the results of Section 4 to the super case, showing how an even $p$-cocycle on a nilpotent Lie superalgebra can be integrated to a smooth $p$-cocycle on the corresponding supergroup. Finally, in Section 8, we apply this technique to construct the superstring Lie 2-supergroup, Superstring $(n+1,1)$, in the guise of a smooth 3-cocycle on the Poincaré supergroup. 


\section{Lie 2-groups from group cohomology}

Roughly speaking, an " $n$-group" is a weak $n$-groupoid with one object-an $n$-category with one object in which all morphisms are weakly invertible, up to higher-dimensional morphisms. This definition is a rough one because there are many possible definitions to use for "weak $n$-category", but despite this ambiguity, it can still serve to motivate us.

The richness of weak $n$-categories, no matter what definition we apply, makes $n$-groups a complicated subject. In the midst of this complexity, we seek to define a class of $n$-groups that have a simple description, and which are straightforward to internalize, so that we may easily construct Lie $n$-groups and Lie $n$-supergroups, as we shall do later in this paper. The motivating example for this is what Baez and Lauda [8] call a "special 2-group", which has a concrete description using group cohomology. Since Baez and Lauda prove that all 2-groups are equivalent to special ones, group cohomology also serves to classify 2-groups.

So, we will define "slim Lie $n$-groups" precisely only for $n=2$, but sketch a definition for higher $n$. This is an Lie $n$-group which is skeletal (every weakly isomorphic pair of objects are equal), and almost trivial: all $k$-morphisms are the identity for $1<k<n$. Slim Lie $n$-groups are useful because they can be completely classified by Lie group cohomology. They are also easy to "superize", and their super versions can be completely classified using Lie supergroup cohomology, as we shall see in Section 6. Finally, we note that we could equally well-define "slim $n$-groups", working in the category of sets rather than the category of smooth manifolds. Indeed, when $n=2$, this is what Baez and Lauda call a "special 2-group", though we prefer the "slim" terminology.

We should stress that the definition of Lie $n$-group we sketch here (and make precise for $n \leq 3$ ), while it is good enough for our needs, is known to be too naive in some important respects. For instance, it does not seem possible to integrate every Lie $n$-algebra to a Lie $n$-group of this type, while Henriques's definition of Lie $n$-group does make this possible [31].

First we need to review the cohomology of Lie groups, as originally defined by van Est [55], who was working in parallel with the definition of group cohomology given by Eilenberg and MacLane. Fix a Lie group $G$, an abelian Lie group $H$, and a smooth action of $G$ on $H$ which respects addition in $H$. That is, for any $g \in G$ and $h, h^{\prime} \in H$, we have:

$$
g\left(h+h^{\prime}\right)=g h+g h^{\prime} .
$$


Then the cohomology of $\boldsymbol{G}$ with coefficients in $\boldsymbol{H}$ is given by the Lie group cochain complex, $C^{\bullet}(G, H)$. At level $p$, this consists of the smooth functions from $G^{p}$ to $H$ :

$$
C^{p}(G, H)=\left\{f: G^{p} \rightarrow H\right\} .
$$

We call elements of this set $\boldsymbol{H}$-valued $\boldsymbol{p}$-cochains on $\boldsymbol{G}$. The boundary operator is the same as the one defined by Eilenberg-MacLane. On a $p$-cochain $f$, it is given by the formula:

$$
\begin{aligned}
d f\left(g_{1}, \ldots, g_{p+1}\right)= & g_{1} f\left(g_{2}, \ldots, g_{p+1}\right) \\
& +\sum_{i=1}^{p}(-1)^{i} f\left(g_{1}, \ldots, g_{i-1}, g_{i} g_{i+1}, g_{i+2}, \ldots, g_{p+1}\right) \\
& +(-1)^{p+1} f\left(g_{1}, \ldots, g_{p}\right) .
\end{aligned}
$$

The proof that $d^{2}=0$ is routine. All the usual terminology applies: a $p$ cochain $f$ for which $d f=0$ is called closed, or a cocycle, a $p$-cochain $f=d g$ for some $(p-1)$-cochain $g$ is called exact, or a coboundary. A $p$-cochain is said to be normalized if it vanishes when any of its entries is 1. Every cohomology class can be represented by a normalized cocycle. Finally, when $H=\mathbb{R}$ with trivial $G$ action, we omit it when writing the complex $C^{\bullet}(G)$, and we call real-valued cochains, cocycles, or coboundaries, simply cochains, cocycles or coboundaries, respectively.

This last choice, that $\mathbb{R}$ will be our default coefficient group, may seem innocuous, but there is another one-dimensional abelian Lie group we might have chosen: $\mathrm{U}(1)$, the group of phases. This would have been an equally valid choice, and perhaps better for some physical applications, but we have chosen $\mathbb{R}$ because it simplifies our formulas slightly.

We now sketch how to build a slim Lie $n$-group from an $(n+1)$-cocycle. In essence, given a normalized $H$-valued $(n+1)$-cocycle $a$ on a Lie group $G$, we want to construct a Lie $n$-group $\operatorname{Brane}_{a}(G, H)$, which is the smooth, weak $n$-groupoid with:

- One object. We can depict this with a dot, or "0-cell":

- For each element $g \in G$, a 1-automorphism of the one object, which we depict as an arrow, or "1-cell":

$$
\text { - } \stackrel{g}{\longrightarrow} \bullet, \quad g \in G .
$$

Composition corresponds to multiplication in the group:

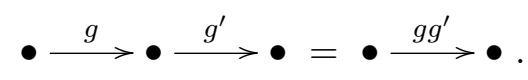


- Trivial $k$-morphisms for $1<k<n$. If we depict 2-morphisms with 2cells, 3-morphisms with 3-cells, then we are saying there is just one of each of these (the identity) up to level $n-1$ :

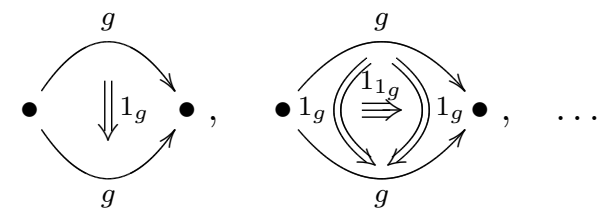

- For each element $h \in H$, an $n$-automorphism on the identity of the identity of ...the identity of the 1-morphism $g$, and no $n$-morphisms which are not $n$-automorphisms. For example, when $n=3$, we have:

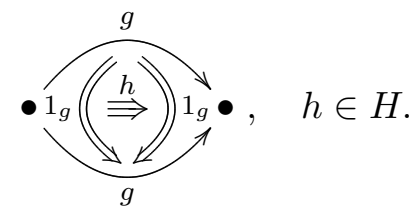

- There are $n$ ways of composing $n$-morphisms, given by different ways of sticking $n$-cells together. For example, when $n=3$, we can glue two 3 -cells along a 2-cell, which should just correspond to addition in $H$ :

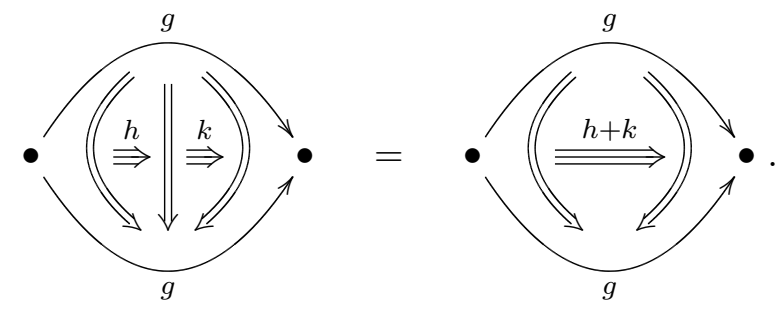

We also can glue two 3-cells along a 1-cell, which should again just be addition in $H$ :

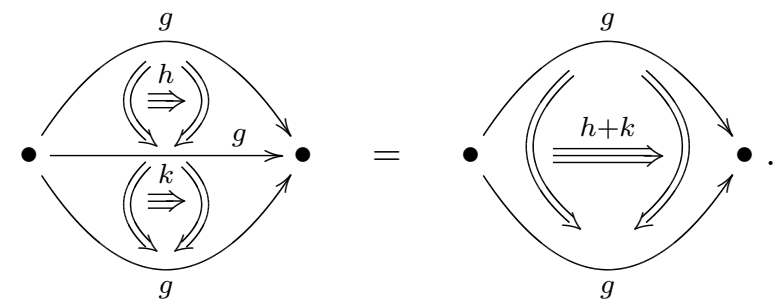

And finally, we can glue two 3-cells at the 0-cell, the object $\bullet$. This is the only composition of $n$-morphisms where the attached 1-morphisms 
"ATMP-16-5-A4-HUE" — 2013/4/30 — 17:32 — page 1496 — \#12

can be distinct, which distinguishes it from the first two cases. It should be addition twisted by the action of $G$ :
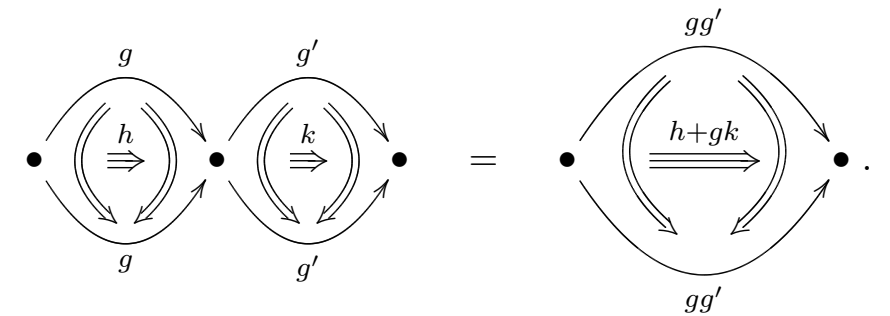

For arbitary $n$, we define all $n$ compositions to be addition in $H$, except for gluing at the object, where it is addition twisted by the action.

- For any $(n+1)$-tuple of 1-morphisms, an $n$-automorphism $a\left(g_{1}, g_{2}, \ldots, g_{n+1}\right)$ on the identity of the identity of ...the identity of the 1-morphism $g_{1} g_{2} \ldots g_{n+1}$. We call $a$ the $\boldsymbol{n}$-associator.

- $a$ satisfies an equation corresponding to the $n$-dimensional associahedron, which is equivalent to the cocycle condition.

In principle, it should be possible to take a globular definition of $n$-category, such as that of Batanin or Trimble, and fill out this sketch to make it a real definition of an $n$-group. Doing this here, however, would lead us too far afield from our goal, for which we only need 2-groups. So let us flesh out this case. The reader interested in learning more about the various definitions of $n$-categories should consult Leinster's survey [37] or Cheng and Lauda's guidebook [16].

\section{$2.1 \quad$ Lie 2-groups}

Speaking precisely, a 2-group is a bicategory with one object in which all 1-morphisms and 2-morphisms are weakly invertible. Rather than plain 2 -groups, we are interested in Lie 2-groups, where all the structure in sight is smooth. So, we really need a bicategory "internal to the category of smooth manifolds", or a "smooth bicategory". To this end, we will give an especially long and unfamiliar definition of bicategory, isolating each operation and piece of data so that we can indicate its smoothness. Readers not familiar with bicategories are encouraged to read the introduction by Leinster [36].

Before we give this definition, let us review the idea of a "bicategory", so that its basic simplicity is not obscured in technicalities. A bicategory has objects: 
"ATMP-16-5-A4-HUE" — 2013/4/30 — 17:32 — page 1497 — \#13

morphisms going between objects,

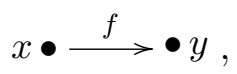

and 2-morphisms going between morphisms:

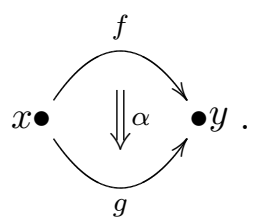

Morphisms in a bicategory can be composed just as morphisms in a category:

$$
x \stackrel{f}{\longrightarrow} y \stackrel{g}{\longrightarrow} z=\quad x \stackrel{f \cdot g}{\longrightarrow} z .
$$

But there are two ways to compose 2-morphisms — vertically:

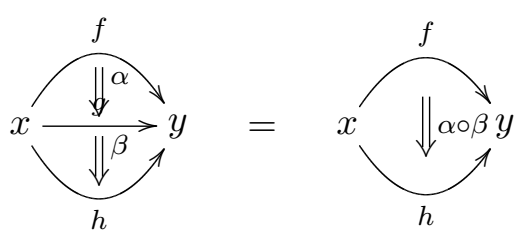

and horizontally:

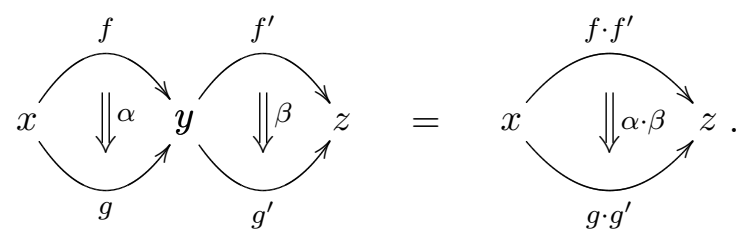

Unlike a category, composition of morphisms need not be associative or have left and right units. The presence of 2-morphisms allows us to weaken the axioms. Rather than demanding $(f \cdot g) \cdot h=f \cdot(g \cdot h)$, for composable morphisms $f, g$ and $h$, the presence of 2 -morphisms allows for the weaker condition that these two expressions are merely isomorphic:

$$
a(f, g, h):(f \cdot g) \cdot h \Rightarrow f \cdot(g \cdot h),
$$


where $a(f, g, h)$ is an 2-isomorphism called the associator. In the same vein, rather than demanding that:

$$
1_{x} \cdot f=f=f \cdot 1_{y},
$$

for $f: x \rightarrow y$, and identities $1_{x}: x \rightarrow x$ and $1_{y}: y \rightarrow y$, the presence of 2-morphisms allows us to weaken these equations to isomorphisms:

$$
l(f): 1_{x} \cdot f \Rightarrow f, \quad r(f): f \cdot 1_{y} \Rightarrow f .
$$

Here, $l(f)$ and $r(f)$ are 2-isomorphisms called the left and right unitors.

Of course, these 2-isomorphisms obey rules of their own. The associator satisfies its own axiom, called the pentagon identity, which says that this pentagon commutes:

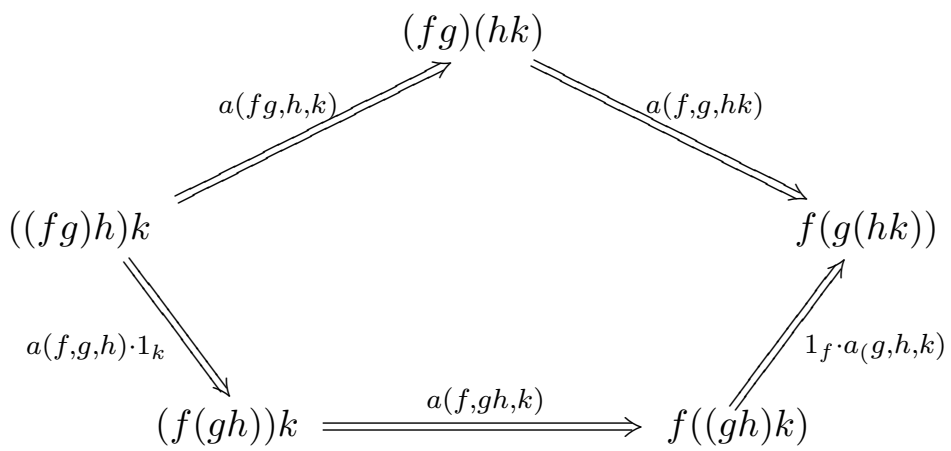

Finally, the associator and left and right unitors satisfy the triangle identity, which says the following triangle commutes:

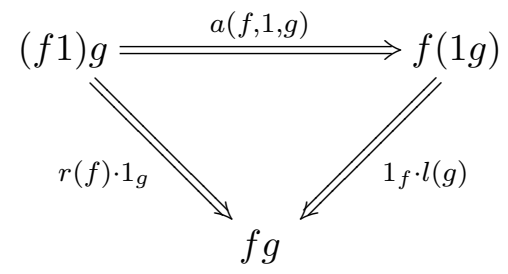

A word of caution is needed here before we proceed: in this section only, we are bucking standard mathematical practice by writing the result of doing first $\alpha$ and then $\beta$ as $\alpha \circ \beta$ rather than $\beta \circ \alpha$, as one would do in most contexts where $\circ$ denotes composition of functions. This has the effect of 
changing how we read commutative diagrams. For instance, the commutative triangle:

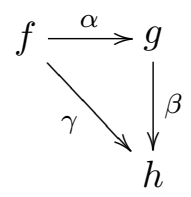

reads $\gamma=\alpha \circ \beta$ rather than $\gamma=\beta \circ \alpha$.

We shall now give the full definition, not of a bicategory, but of a "smooth bicategory". To do this, we use the idea of internalization. Dating back to Ehresmann [22] in the 1960s, internalization has become a standard tool of the working category theorist. The idea is based on a familiar one: any mathematical structure that can be defined using sets, functions, and equations between functions can often be defined in categories other than Set. For instance, a group in the category of smooth manifolds is a Lie group. To perform internalization, we apply this idea to the definition of category itself. We recall the essentials here to define "smooth categories". More generally, one can define a "category in $K$ " for many categories $K$, though here we will work exclusively with the example where $K$ is the category of smooth manifolds. For a readable treatment of internalization, see Borceux's handbook [13].

Definition 1. A smooth category $C$ consists of

- a smooth manifold of objects $C_{0}$;

- a smooth manifold of morphisms $C_{1}$;

together with

- smooth source and target maps $s, t: C_{1} \rightarrow C_{0}$,

- a smooth identity-assigning map $i: C_{0} \rightarrow C_{1}$,

- a smooth composition map o: $C_{1} \times{ }_{C_{0}} C_{1} \rightarrow C_{1}$, where $C_{1} \times{ }_{C_{0}} C_{1}$ is the pullback of the source and target maps:

$$
C_{1} \times C_{0} C_{1}=\left\{(f, g) \in C_{1} \times C_{1}: t(f)=s(g)\right\},
$$

and is assumed to be a smooth manifold.

such that the following diagrams commute, expressing the usual category laws: 
"ATMP-16-5-A4-HUE" — 2013/4/30 — 17:32 — page 1500 — \#16

- laws specifying the source and target of identity morphisms:
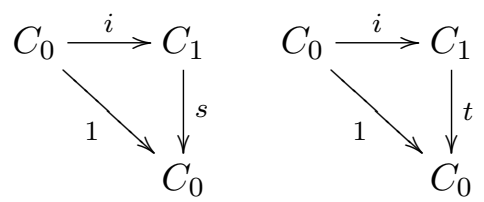

- laws specifying the source and target of composite morphisms:
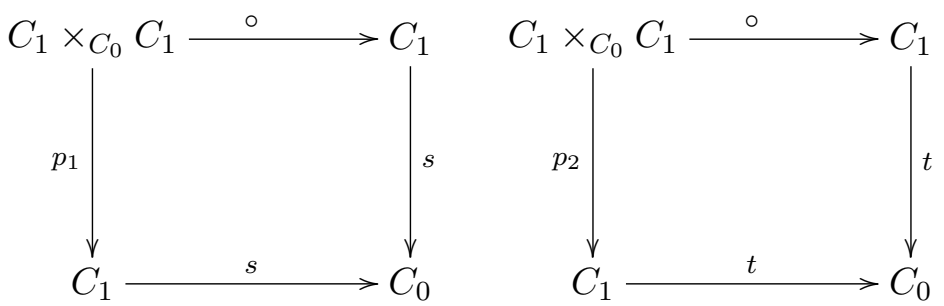

- the associative law for composition of morphisms:

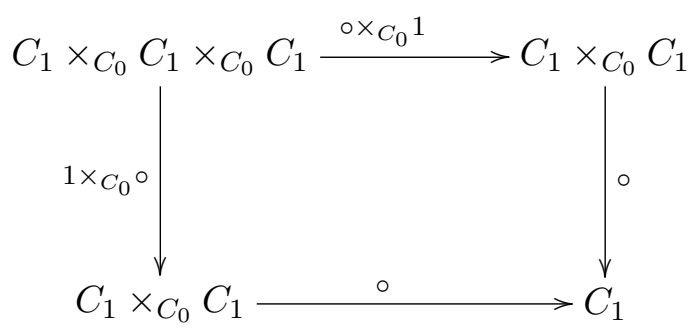

- the left and right unit laws for composition of morphisms:

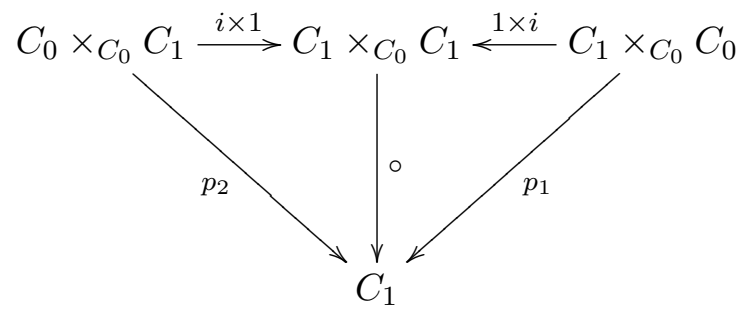

The existence of pullbacks in the category of smooth manifolds is a delicate issue. When working with categories internal to some category $K$, it is customary to assume $K$ contains all pullbacks, but this is merely a convenience. All the definitions still work as long as the existence of each required pullback is implicit. 
To define smooth bicategories, we must first define smooth functors and natural transformations:

Definition 2. Given smooth categories $C$ and $C^{\prime}$, a smooth functor $F: C \rightarrow C^{\prime}$ consists of:

- a smooth map on objects, $F_{0}: C_{0} \rightarrow C_{0}^{\prime}$;

- a smooth map on morphisms, $F_{1}: C_{1} \rightarrow C_{1}^{\prime}$;

such that the following diagrams commute, corresponding to the usual laws satisfied by a functor:

- preservation of source and target:
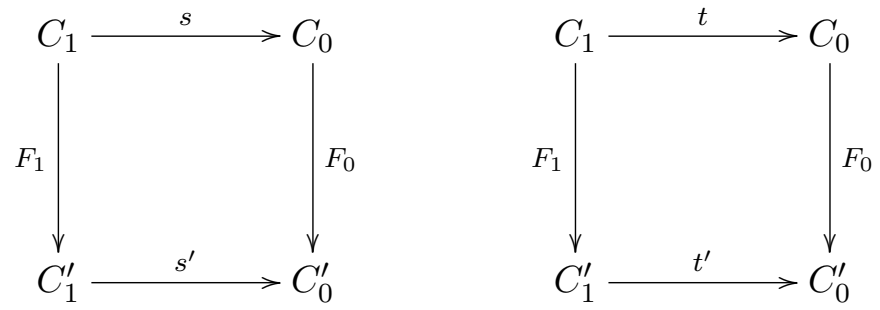

- preservation of identity morphisms:

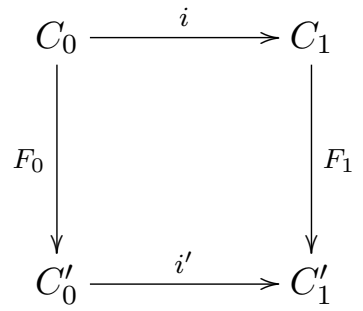

- preservation of composite morphisms:

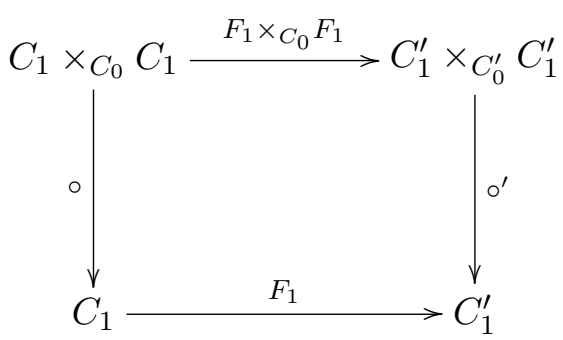

Definition 3. Given smooth categories $C$ and $C^{\prime}$, and smooth functors $F, G: C \rightarrow C^{\prime}$, a smooth natural transformation $\theta: F \Rightarrow G$ is a smooth 
"ATMP-16-5-A4-HUE" — 2013/4/30 — 17:32 — page 1502 — \#18

map $\theta: C_{0} \rightarrow C_{1}^{\prime}$ for which the following diagrams commute, expressing the usual laws satisfied by a natural transformation:

- laws specifying the source and target of the natural transformation:
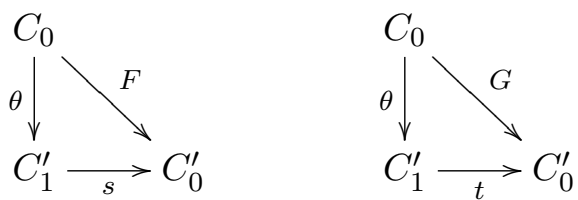

- the commutative square law:

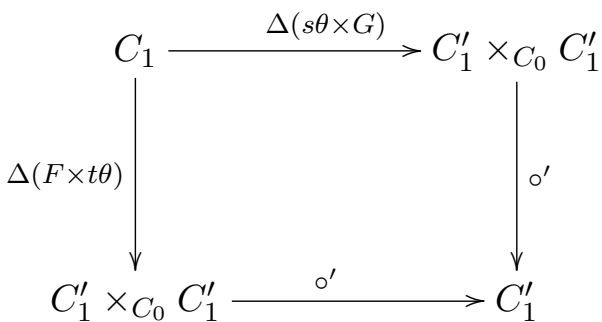

Given a third smooth functor $H: C \rightarrow C^{\prime}$ and a smooth natural transformation $\eta: G \Rightarrow H$, we define the composition $\theta \eta: F \Rightarrow H$ to be the smooth map:

$$
C_{0} \stackrel{\Delta(\theta \times \eta)}{\longrightarrow} C_{1}^{\prime} \times_{C_{0}^{\prime}} C_{1}^{\prime} \stackrel{\circ}{\longrightarrow} C_{1}^{\prime}
$$

The identity natural transformation $1_{F}: F \Rightarrow F$ on a smooth functor $F: C \rightarrow C^{\prime}$ is defined to be the smooth map:

$$
C_{0} \stackrel{i}{\longrightarrow} C_{1} \stackrel{F_{1}}{\longrightarrow} C_{1}^{\prime}
$$

where $i$ is the identity-assigning map for $C$ and $F_{1}$ is the component of $F$ on morphisms. Noting that $1_{F}$ acts as a left and right identity under composition of natural transformations, we say that a smooth natural transformation is a smooth natural isomorphism if it has a left and right inverse.

Now we know enough about smooth category theory to bootstrap the definition of smooth bicategories. We do this in a somewhat nonstandard way: we make use of the fact that the morphisms and 2-morphisms of a bicategory form an ordinary category under vertical composition. Generalizing this, the morphisms and 2-morphisms in a smooth bicategory should 
"ATMP-16-5-A4-HUE" — 2013/4/30 — 17:32 — page 1503 — \#19

form, by themselves, a smooth category. We can then define horizontal composition as a smooth functor, and introduce the associator and left and right unitors as smooth natural transformations between certain functors. In detail:

Definition 4. A smooth bicategory $B$ consists of

- a manifold of objects $B_{0}$;

- a manifold of morphisms $B_{1}$;

- a manifold of 2-morphisms $B_{2}$;

equipped with:

- a smooth category structure on $\operatorname{Mor} B$, with

- $B_{1}$ as the smooth manifold of objects;

- $B_{2}$ as the smooth manifold of morphisms;

The composition in $\operatorname{Mor} B$ is called vertical composition and denoted $\circ$.

- smooth source and target maps:

$$
s, t: B_{1} \rightarrow B_{0} .
$$

- a smooth identity-assigning map:

$$
i: B_{0} \rightarrow B_{1} \text {. }
$$

- a smooth horizontal composition functor:

$$
\therefore \underline{\operatorname{Mor}} B \times B_{0} \underline{\operatorname{Mor}} B \rightarrow \underline{\operatorname{Mor}} B .
$$

That is, a pair of smooth maps:

$$
\begin{aligned}
& \cdot: B_{1} \times_{B_{0}} B_{1} \rightarrow B_{1} \\
& \cdot: B_{2} \times_{B_{0}} B_{2} \rightarrow B_{2},
\end{aligned}
$$

satisfying the axioms for a functor.

- a smooth natural isomorphism, the associator:

$$
a(f, g, h):(f \cdot g) \cdot h \Rightarrow f \cdot(g \cdot h) .
$$


"ATMP-16-5-A4-HUE" — 2013/4/30 — 17:32 — page $1504-\# 20$

- smooth natural isomorphisms, the left and right unitors, which are both trivial in the bicategories we consider:

$$
l(f): 1 \cdot f \Rightarrow f, \quad r(f): f \cdot 1 \Rightarrow f .
$$

such that the following diagrams commute, expressing the same laws regarding sources, targets and identities as with a smooth category, and two new laws expressing the compatibility of the various source and target maps:

- laws specifying the source and target of identity morphisms:
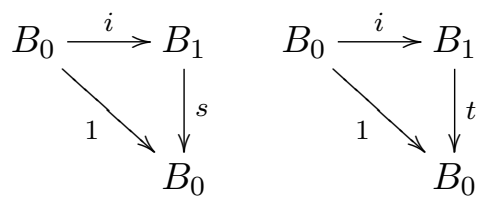

- laws specifying the source and target of the horizontal composite of 1-morphisms:
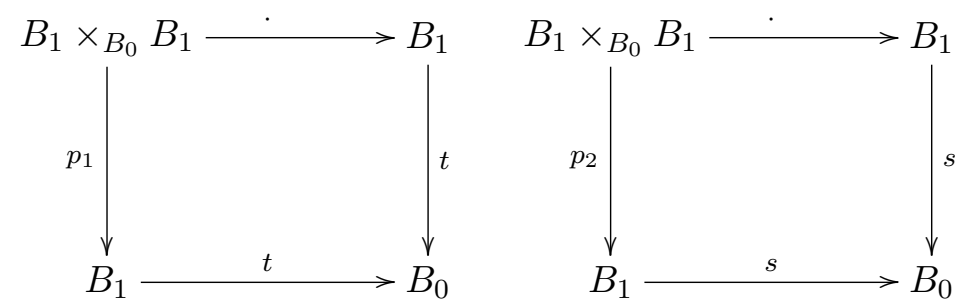

- laws expressing the compatibility of source and target maps:
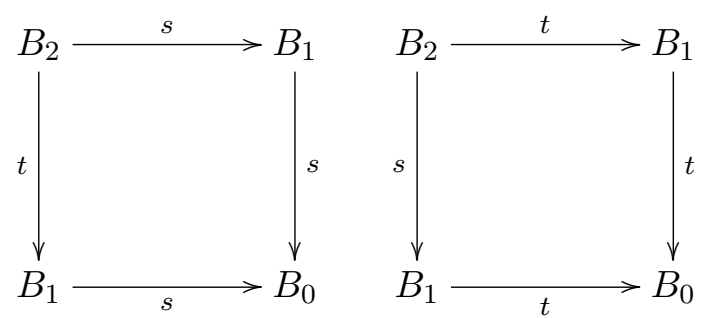

Finally, associator and left and right unitors satisfy some laws of their ownthe following diagrams commute: 
- the pentagon identity for the associator:

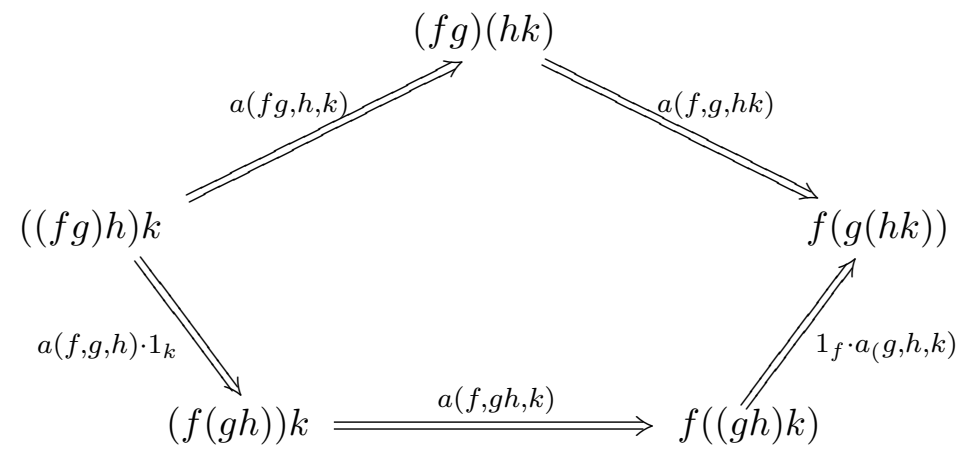

for any four composable morphisms $f, g, h$ and $k$.

- the triangle identity for the left and right unit laws:

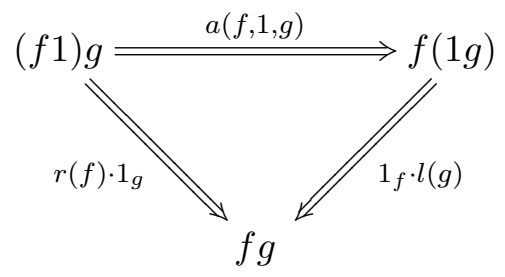

for any two composable morphisms $f$ and $g$.

Finally, to talk about Lie 2-groups, we will need to talk about inverses. We say that the 2-morphisms in a smooth bicategory $B$ have smooth strict inverses if there exists a smooth map from 2-morphisms to 2-morphisms:

$$
\operatorname{inv}_{2}: B_{2} \rightarrow B_{2}
$$

that assigns to each 2-morphism $\alpha$ its strict inverse $\alpha^{-1}=\operatorname{inv}_{2}(\alpha)$, obeying the left and right inverse laws on the nose:

$$
\alpha^{-1} \circ \alpha=1, \quad \alpha \circ \alpha^{-1}=1 .
$$

Of course, if the strict inverse $\alpha^{-1}$ exists, it is unique, but same is not true for "weak inverses". We say that the morphisms in $B$ have smooth weak inverses if there exist smooth maps:

$$
\operatorname{inv}_{1}: B_{1} \rightarrow B_{1}, \quad e: B_{1} \rightarrow B_{2}, \quad u: B_{1} \rightarrow B_{2}
$$


such that for each morphism $f$, inv 1 provides a smooth choice of weak inverse, $f^{-1}=\operatorname{inv}_{1}(f)$, and $u$ and $e$ provide smooth choices of 2 -isomorphisms that "weaken" the left and right inverse laws:

$$
e(f): f^{-1} \cdot f \Rightarrow 1, \quad u(f): f \cdot f^{-1} \Rightarrow 1 .
$$

Here we have been careful to use indefinite articles, and with good reason: unlike their strict counterparts, weak inverses need not be unique!

The definition of smooth bicategory we give above may seem so long that checking it is utterly intimidating, but we shall see an example in a moment where this is easy. This will be an example of a Lie 2-group, a smooth bicategory with one object whose morphisms have smooth weak inverses and whose 2-morphisms have smooth strict inverses.

Secretly, the pentagon identity is a cocycle condition, as we shall now see. Given a normalized $H$-valued 3 -cocycle $a$ on a Lie group $G$, we can construct a Lie 2-group $\operatorname{String}_{a}(G, H)$ with:

- One object, $\bullet$, regarded as a manifold in the trivial way.

- For each element $g \in G$, an automorphism of the one object:

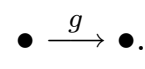

Horizontal composition given by multiplication in the group:

$$
\cdot G \times G \rightarrow G
$$

Note that source and target maps are necessarily trivial. The identityassigning map takes the one object to $1 \in G$.

- For each $h \in H$, a 2-automorphism of the morphism $g$, and no 2-morphisms between distinct morphisms:

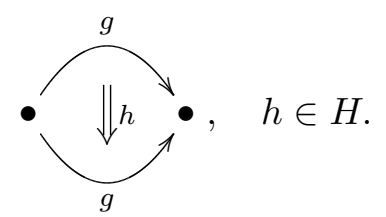

Thus the space of all 2-morphisms is $G \times H$, and the source and target maps are projection onto the first factor. The identity-assigning map takes each element of $G$ to $0 \in H$. 
- Two kinds of composition of 2-morphisms: given a pair of 2-morphisms on the same morphism, vertical composition is given by addition in $H$ :

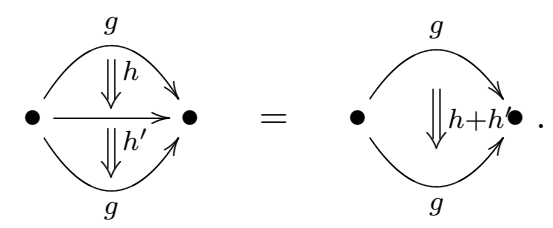

That is, vertical composition is just the map:

$$
\circ=1 \times+: G \times H \times H \rightarrow G \times H .
$$

where we have used the fact that the pullback of 2-morphisms over the one object is trivially:

$$
(G \times H) \times \bullet(G \times H) \cong G \times H \times H .
$$

Given a pair of 2-morphisms on different morphisms, horizontal composition is addition twisted by the action of $G$ :

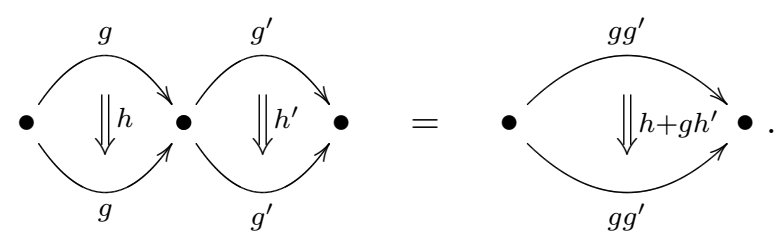

Or, in terms of a map, this is the multiplication on the semidirect product, $G \ltimes H$ :

$$
\because(G \ltimes H) \times(G \ltimes H) \rightarrow G \ltimes H .
$$

- For any triple of morphisms, a 2-isomorphism, the associator:

$$
a\left(g_{1}, g_{2}, g_{3}\right): g_{1} g_{2} g_{3} \rightarrow g_{1} g_{2} g_{3},
$$

given by the 3 -cocycle $a$ : $G^{3} \rightarrow H$, where by a slight abuse of definitions we think of this 2-isomorphism as living in $H$ rather than $G \times H$, because the source (and target) are understood to be $g_{1} g_{2} g_{3}$.

- The left and right unitors are trivial.

A slim Lie 2-group is one of this form. When $H=\mathbb{R}$, we write simply $\operatorname{String}_{a}(G)$ for the above Lie 2-group. It remains to check that this is, in fact, a Lie 2-group: 
Proposition 5. String $(G, H)$ is a Lie 2-group: a smooth bicategory with one object in which all morphisms have smooth weak inverses and all 2-morphisms have smooth strict inverses.

In brief, we prove this by showing that the 3-cocycle condition implies the one nontrivial axiom for this bicategory: the pentagon identity.

Proof. First, let us dispense with the easier items from our definition. For String $_{a}(G, H)$, it is easy to see that the morphisms and 2-morphism form a smooth category under vertical composition, that horizontal composition is a smooth functor, and that the associator defines a natural transformation. Since the left and right unitors are the identity, the triangle identity just says $a\left(g_{1}, 1, g_{2}\right)=1$. Or, written additively, $a\left(g_{1}, 1, g_{2}\right)=0$. Because $a$ is normalized, this is automatic.

To check that $\operatorname{String}_{a}(G, H)$ is really a bicategory, it therefore remains to check the pentagon identity. This says that the following automorphisms of $g_{1} g_{2} g_{3} g_{4}$ are equal:

$$
\begin{aligned}
a\left(g_{1}, g_{2}, g_{3} g_{4}\right) \circ a\left(g_{1} g_{2}, g_{3}, g_{4}\right)= & \left(1_{g_{1}} \cdot a\left(g_{2}, g_{3}, g_{4}\right)\right) \circ a\left(g_{1}, g_{2} g_{3}, g_{4}\right) \\
& \circ\left(a\left(g_{1}, g_{2}, g_{3}\right) \cdot 1_{g_{4}}\right) .
\end{aligned}
$$

Or, using the definition of vertical composition:

$$
\begin{aligned}
a\left(g_{1}, g_{2}, g_{3} g_{4}\right)+a\left(g_{1} g_{2}, g_{3}, g_{4}\right)= & \left(1_{g_{1}} \cdot a\left(g_{2}, g_{3}, g_{4}\right)\right)+a\left(g_{1}, g_{2} g_{3}, g_{4}\right) \\
& +\left(a\left(g_{1}, g_{2}, g_{3}\right) \cdot 1_{g_{4}}\right) .
\end{aligned}
$$

Finally, use the definition of the dot operation for 2-morphisms, as the semidirect product:

$$
\begin{aligned}
a\left(g_{1}, g_{2}, g_{3} g_{4}\right)+a\left(g_{1} g_{2}, g_{3}, g_{4}\right)= & \left.g_{1} a\left(g_{2}, g_{3}, g_{4}\right)\right)+a\left(g_{1}, g_{2} g_{3}, g_{4}\right) \\
& +a\left(g_{1}, g_{2}, g_{3}\right) .
\end{aligned}
$$

This is the 3-cocycle condition - it holds because $a$ is a 3-cocycle.

So, $\operatorname{String}_{a}(G, H)$ is a bicategory. It is smooth because everything in sight is smooth: $G, H$, the source, target, identity-assigning, and composition maps, and the associator $a: G^{3} \rightarrow H$. And it is a Lie 2-group: the morphisms in $G$ and 2-morphisms in $H$ all have smooth strict inverses given by inversion in the Lie groups $G$ and $H$.

In fact, we can say something a bit stronger about $\operatorname{String}_{a}(G, H)$, if we let $a$ be any normalized $H$-valued 3-cochain, rather requiring it to be a cocycle. 
In this case, $\operatorname{String}_{a}(G, H)$ is a Lie 2-group if and only if $a$ is a 3-cocycle, because $a$ satisfies the pentagon identity if and only if it is a cocycle.

\section{Lie $n$-superalgebras from Lie superalgebra cohomology}

Having sketched the construction of Lie $n$-groups from smooth group $(n+1)$-cocycles, we now turn to a parallel construction of Lie $n$-algebras. As one might expect from experience with ordinary Lie groups and Lie algebras, Lie $n$-algebras are much easier than their Lie $n$-group counterparts, and it is straightforward to give the definition for all $n$. It also straightforward to incorporate the "super" case immediately.

As we touched on in the Introduction, a Lie $n$-superalgebra is a certain kind of $L_{\infty}$-superalgebra, which is the super version of an $L_{\infty}$-algebra. This last is a chain complex, $V$ :

$$
V_{0} \stackrel{d}{\longleftarrow} V_{1} \stackrel{d}{\longleftarrow} V_{2} \stackrel{d}{\longleftarrow} \cdots
$$

equipped with a structure like that of a Lie algebra, but where the Jacobi identity only holds up to chain homotopy, and this chain homotopy satisfies its own identity up to chain homotopy, and so on. For an $L_{\infty}$-superalgebra, each term in the chain complex has a $\mathbb{Z}_{2}$-grading, and we introduce extra signs. A Lie $n$-superalgebra is an $L_{\infty}$-superalgebra in which only the first $n$ terms are nonzero, starting with $V_{0}$.

In the last section, we sketched how a Lie group $(n+1)$-cocycle:

$$
f: G^{n} \rightarrow H
$$

can be used to construct an especially simple Lie $n$-group, with 1-morphisms forming the group $G, n$-morphisms forming the group $G \ltimes H$, and all $k$-morphisms in between trivial. In this section, we shall describe how a Lie superalgebra $(n+1)$-cocycle:

$$
\omega: \Lambda^{n} \mathfrak{g} \rightarrow \mathfrak{h}
$$

can be used to construct an especially simple Lie $n$-superalgebra, defined on a chain complex with $\mathfrak{g}$ in grade $0, \mathfrak{h}$ in grade $n-1$, and all terms in between trivial. To make this precise, we had better start with some definitions.

To begin at the beginning, a super vector space is a $\mathbb{Z}_{2}$-graded vector space $V=V_{0} \oplus V_{1}$ where $V_{0}$ is called the even part, and $V_{1}$ is called the odd part. There is a symmetric monoidal category SuperVect which has: 
"ATMP-16-5-A4-HUE" — 2013/4/30 — 17:32 — page 1510 — \#26

- $\mathbb{Z}_{2}$-graded vector spaces as objects;

- Grade-preserving linear maps as morphisms;

- A tensor product $\otimes$ that has the following grading: if $V=V_{0} \oplus V_{1}$ and $W=W_{0} \oplus W_{1}$, then $(V \otimes W)_{0}=\left(V_{0} \otimes W_{0}\right) \oplus\left(V_{1} \otimes W_{1}\right)$ and $(V \otimes W)_{1}=\left(V_{0} \otimes W_{1}\right) \oplus\left(V_{1} \otimes W_{0}\right) ;$

- A braiding

$$
B_{V, W}: V \otimes W \rightarrow W \otimes V
$$

defined as follows: $v \in V$ and $w \in W$ are of grade $|v|$ and $|w|$, then

$$
B_{V, W}(v \otimes w)=(-1)^{|v||w|} w \otimes v .
$$

The braiding encodes the "the rule of signs": in any calculation, when two odd elements are interchanged, we introduce a minus sign. We can see this in the axioms of a Lie superalgebra, which resemble those of a Lie algebra with some extra signs.

Briefly, a Lie superalgebra $\mathfrak{g}$ is a Lie algebra in the category of super vector spaces. More concretely, it is a super vector space $\mathfrak{g}=\mathfrak{g}_{0} \oplus \mathfrak{g}_{1}$, equipped with a graded-antisymmetric bracket:

$$
[-,-]: \Lambda^{2} \mathfrak{g} \rightarrow \mathfrak{g},
$$

which satisfies the Jacobi identity up to signs:

$$
[X,[Y, Z]]=[[X, Y], Z]+(-1)^{|X||Y|}[Y,[X, Z]]
$$

for all homogeneous $X, Y, Z \in \mathfrak{g}$. Note how we have introduced an extra minus sign upon interchanging $X$ and $Y$, exactly as the rule of signs says we should.

It is straightforward to generalize the cohomology of Lie algebras, as defined by Chevalley-Eilenberg [17,1], to Lie superalgebras [38]. Suppose $\mathfrak{g}$ is a Lie superalgebra and $\mathfrak{h}$ is a representation of $\mathfrak{g}$. That is, $\mathfrak{h}$ is a supervector space equipped with a Lie superalgebra homomorphism $\rho: \mathfrak{g} \rightarrow \mathfrak{g l}(\mathfrak{h})$. The cohomology of $\mathfrak{g}$ with coefficients in $\mathfrak{h}$ is computed using the Lie superalgebra cochain complex, which consists of graded-antisymmetric $p$-linear maps at level $p$ :

$$
C^{p}(\mathfrak{g}, \mathfrak{h})=\left\{\omega: \Lambda^{p} \mathfrak{g} \rightarrow \mathfrak{h}\right\} .
$$

We call elements of this set $\mathfrak{h}$-valued $\boldsymbol{p}$-cochains on $\mathfrak{g}$. Note that the $C^{p}(\mathfrak{g}, \mathfrak{h})$ is a super vector space, in which grade-preserving elements are 
even, while grade-reversing elements are odd. When $\mathfrak{h}=\mathbb{R}$, the trivial representation, we typically omit it from the cochain complex and all associated groups, such as the cohomology groups. Thus, we write $C^{\bullet}(\mathfrak{g})$ for $C^{\bullet}(\mathfrak{g}, \mathbb{R})$.

Next, we define the coboundary operator $d: C^{p}(\mathfrak{g}, \mathfrak{h}) \rightarrow C^{p+1}(\mathfrak{g}, \mathfrak{h})$. Let $\omega$ be a homogeneous $p$-cochain and let $X_{1}, \ldots, X_{p+1}$ be homogeneous elements of $\mathfrak{g}$. Now define:

$$
\begin{aligned}
d \omega & \left(X_{1}, \ldots, X_{p+1}\right) \\
= & \sum_{i=1}^{p+1}(-1)^{i+1}(-1)^{\left|X_{i}\right||\omega|} \epsilon_{1}^{i-1}(i) \rho\left(X_{i}\right) \omega\left(X_{1}, \ldots, \hat{X}_{i}, \ldots, X_{p+1}\right) \\
& +\sum_{i<j}(-1)^{i+j}(-1)^{\left|X_{i}\right|\left|X_{j}\right|} \epsilon_{1}^{i-1}(i) \epsilon_{1}^{j-1}(j) \\
& \quad \times \omega\left(\left[X_{i}, X_{j}\right], X_{1}, \ldots, \hat{X}_{i}, \ldots, \hat{X}_{j}, \ldots X_{p+1}\right) .
\end{aligned}
$$

Here, $\epsilon_{i}^{j}(k)$ is shorthand for the sign one obtains by moving $X_{k}$ through $X_{i}, X_{i+1}, \ldots, X_{j}$. In other words,

$$
\epsilon_{i}^{j}(k)=(-1)^{\left|X_{k}\right|\left(\left|X_{i}\right|+\left|X_{i+1}\right|+\cdots+\left|X_{j}\right|\right)} .
$$

Following the usual argument for Lie algebras, one can check that:

Proposition 6. The Lie superalgebra coboundary operator d satisfies $d^{2}=0$.

We thus say an $\mathfrak{h}$-valued $p$-cochain $\omega$ on $\mathfrak{g}$ is a $\boldsymbol{p}$-cocycle or closed when $d \omega=0$, and a $\boldsymbol{p}$-coboundary or exact if there exists an $(p-1)$-cochain $\theta$ such that $\omega=d \theta$. Every $p$-coboundary is a $p$-cocycle, and we say an $p$-cocycle is trivial if it is a coboundary. We denote the super vector spaces of $p$-cocycles and $p$-coboundaries by $Z^{p}(\mathfrak{g}, \mathfrak{h})$ and $B^{p}(\mathfrak{g}, \mathfrak{h})$ respectively. The $p$ th Lie superalgebra cohomology of $\mathfrak{g}$ with coefficients in $\mathfrak{h}$, denoted $H^{p}(\mathfrak{g}, \mathfrak{h})$ is defined by

$$
H^{p}(\mathfrak{g}, \mathfrak{h})=Z^{p}(\mathfrak{g}, \mathfrak{h}) / B^{p}(\mathfrak{g}, \mathfrak{h})
$$

This super vector space is nonzero if and only if there is a nontrivial $p$-cocycle. In what follows, we shall be especially concerned with the even part of this super vector space, which is nonzero if and only if there is a nontrivial even $p$-cocycle. Our motivation for looking for even cocycles is simple: these parity-preserving maps can regarded as morphisms in the category of 
super vector spaces, which is crucial for the construction in Theorem 8 and everything following it.

Suppose $\mathfrak{g}$ is a Lie superalgebra with a representation on a supervector space $\mathfrak{h}$. Then we shall prove that an even $\mathfrak{h}$-valued $(n+1)$-cocycle $\omega$ on $\mathfrak{g}$ lets us construct an Lie $n$-superalgebra, called $\mathfrak{b r a n} \mathfrak{e}_{\omega}(\mathfrak{g}, \mathfrak{h})$, of the following form:

$$
\mathfrak{g} \stackrel{d}{\longleftarrow} 0 \stackrel{d}{\longleftarrow} \cdots \stackrel{d}{\longleftarrow} 0 \stackrel{d}{\longleftarrow} \mathfrak{h} .
$$

Now let us make all of these ideas precise. In what follows, we shall use super chain complexes, which are chain complexes in the category SuperVect of $\mathbb{Z}_{2}$-graded vector spaces:

$$
V_{0} \stackrel{d}{\longleftarrow} V_{1} \stackrel{d}{\longleftarrow} V_{2} \stackrel{d}{\longleftarrow} \cdots
$$

Thus each $V_{p}$ is $\mathbb{Z}_{2}$-graded and $d$ preserves this grading.

There are thus two gradings in play: the $\mathbb{Z}$-grading by degree, and the $\mathbb{Z}_{2}$-grading on each vector space, which we call the parity. We shall require a sign convention to establish how these gradings interact. If we consider an object of odd parity and odd degree, is it in fact even overall? By convention, we assume that it is. That is, whenever we interchange something of parity $p$ and degree $q$ with something of parity $p^{\prime}$ and degree $q^{\prime}$, we introduce the sign $(-1)^{(p+q)\left(p^{\prime}+q^{\prime}\right)}$. We shall call the sum $p+q$ of parity and degree the overall grade, or when it will not cause confusion, simply the grade. We denote the overall grade of $X$ by $|X|$.

We require a compressed notation for signs. If $x_{1}, \ldots, x_{n}$ are graded, $\sigma \in S_{n}$ a permutation, we define the Koszul sign $\epsilon(\sigma)=\epsilon\left(\sigma ; x_{1}, \ldots, x_{n}\right)$ by

$$
x_{1} \ldots x_{n}=\epsilon\left(\sigma ; x_{1}, \ldots, x_{n}\right) \cdot x_{\sigma(1)} \ldots x_{\sigma(n)},
$$

the sign we would introduce in the free graded-commutative algebra generated by $x_{1}, \ldots, x_{n}$. Thus, $\epsilon(\sigma)$ encodes all the sign changes that arise from permuting graded elements. Now define:

$$
\chi(\sigma)=\chi\left(\sigma ; x_{1}, \ldots, x_{n}\right):=\operatorname{sgn}(\sigma) \cdot \epsilon\left(\sigma ; x_{1}, \ldots, x_{n}\right) .
$$

Thus, $\chi(\sigma)$ is the sign we would introduce in the free graded-anticommutative algebra generated by $x_{1}, \ldots, x_{n}$. 
Yet we shall only be concerned with particular permutations. If $n$ is a natural number and $1 \leq j \leq n-1$ we say that $\sigma \in S_{n}$ is an $(\boldsymbol{j}, \boldsymbol{n}-\boldsymbol{j})$ unshuffle if

$$
\sigma(1) \leq \sigma(2) \leq \cdots \leq \sigma(j) \quad \text { and } \quad \sigma(j+1) \leq \sigma(j+2) \leq \cdots \leq \sigma(n) .
$$

Readers familiar with shuffles will recognize unshuffles as their inverses. A shuffle of two ordered sets (such as a deck of cards) is a permutation of the ordered union preserving the order of each of the given subsets. An unshuffle reverses this process. We denote the collection of all $(j, n-j)$ unshuffles by $S_{(j, n-j)}$.

The following definition of an $L_{\infty}$-algebra was formulated by Schlessinger and Stasheff in 1985 [46]:

Definition 7. An $\boldsymbol{L}_{\infty}$-superalgebra is a graded vector space $V$ equipped with a system $\left\{l_{k} \mid 1 \leq k<\infty\right\}$ of linear maps $l_{k}: V^{\otimes k} \rightarrow V$ with $\operatorname{deg}\left(l_{k}\right)=$ $k-2$, which are totally antisymmetric in the sense that

$$
l_{k}\left(x_{\sigma(1)}, \ldots, x_{\sigma(k)}\right)=\chi(\sigma) l_{k}\left(x_{1}, \ldots, x_{n}\right)
$$

for all $\sigma \in S_{n}$ and $x_{1}, \ldots, x_{n} \in V$, and, moreover, the following generalized form of the Jacobi identity holds for $0 \leq n<\infty$ :

$\sum_{i+j=n+1} \sum_{\sigma \in S_{(i, n-i)}} \chi(\sigma)(-1)^{i(j-1)} l_{j}\left(l_{i}\left(x_{\sigma(1)}, \ldots, x_{\sigma(i)}\right), x_{\sigma(i+1)}, \ldots, x_{\sigma(n)}\right)=0$

where the inner summation is taken over all $(i, n-i)$-unshuffles with $i \geq 1$.

A Lie $n$-superalgebra is an $L_{\infty}$-superalgebra where only the first $n$ terms of the chain complex are nonzero. A slim Lie $\boldsymbol{n}$-superalgebra is a Lie $n$-superalgebra $V$ with only two nonzero terms, $V_{0}$ and $V_{n-1}$, and $d=0$. Given an $\mathfrak{h}$-valued $(n+1)$-cocycle $\omega$ on a Lie superalgebra $\mathfrak{g}$, we can construct a slim Lie $n$-superalgebra $\mathfrak{b r a n e} \mathfrak{e}_{\omega}(\mathfrak{g}, \mathfrak{h})$ with:

- $\mathfrak{g}$ in grade $0, \mathfrak{h}$ in grade $n-1$, and trivial super vector spaces in between,

- $d=0$,

- $l_{2}:(\mathfrak{g} \oplus \mathfrak{h})^{\otimes 2} \rightarrow \mathfrak{g} \oplus \mathfrak{h}$ given by:

- the Lie bracket on $\mathfrak{g} \otimes \mathfrak{g}$,

- the action on $\mathfrak{g} \otimes \mathfrak{h}$,

- zero on $\mathfrak{h} \otimes \mathfrak{h}$, as required by grading. 
- $l_{n+1}:(\mathfrak{g} \oplus \mathfrak{h})^{\otimes(n+1)} \rightarrow \mathfrak{g} \oplus \mathfrak{h}$ given by the cocycle $\omega$ on $\mathfrak{g}^{\otimes(n+1)}$, and zero otherwise, as required by grading,

- all other maps $l_{k}$ zero, as required by grading.

It remains to prove that this is, in fact, a Lie $n$-superalgebra. Indeed, more is true: every slim Lie $n$-superalgebra is precisely of this form.

Theorem 8. $\mathfrak{b r a n e}_{\omega}(\mathfrak{g}, \mathfrak{h})$ is a Lie n-superalgebra. Conversely, every slim Lie n-superalgebra is of form $\mathfrak{b r a n e} \omega(\mathfrak{g}, \mathfrak{h})$ for some Lie superalgebra $\mathfrak{g}$, representation $\mathfrak{h}$, and $\mathfrak{h}$-valued $(n+1)$-cocycle $\omega$ on $\mathfrak{g}$.

Proof. See the proof of Theorem 17 in our previous paper [6], which is a straightforward generalization of the proof found in Baez-Crans [3] to the super case.

For the 2-group $\operatorname{String}_{a}(G, H)$, we noted that $a$ is cocycle if and only if $a$ satisfies the pentagon identity. Likewise, the key to the proof of the above theorem is recognizing that $\omega$ is a Lie superalgebra cocycle if and only if the generalized Jacobi identity, Equation 2, holds. By analogy with 2groups, when $n=2$, we will also write $\mathfrak{s t r i n g} \mathfrak{g}_{\omega}(\mathfrak{g}, \mathfrak{h})$ for the Lie 2-superalgebra constructed from the 3 -cocycle $\omega$, and when $\mathfrak{h}$ is the trivial representation $\mathbb{R}$, we omit it. In the next section, we give some examples of these objects.

\subsection{Examples of slim Lie $n$-superalgebras}

\subsubsection{The string Lie 2-algebra}

For $n \geq 3$, consider the Lie algebra $\mathfrak{s o}(n)$ of infinitesimal rotations of $n$-dimensional Euclidean space. This matrix Lie algebra has Killing form given by the trace, $\langle X, Y\rangle=\operatorname{tr}(X Y)$, and an easy calculation shows that

$$
j=\langle-,[-,-]\rangle
$$

is a 3 -cocycle on $\mathfrak{s o}(n)$. We call $j$ the canonical 3-cocycle on $\mathfrak{s o}(n)$. Using $j$, we get a Lie 2-algebra $\mathfrak{s t r i n g}_{j}(\mathfrak{s o}(n))$, which we denote simply by $\mathfrak{s t r i n g}(n)$. We call this the string Lie 2-algebra. First defined by Baez-Crans [3], it is so-named because it turned out to be intimately related to the string group, String $(n)$, the topological group obtained from $\mathrm{SO}(n)$ by killing the first and third homotopy groups. For a description of this relationship, as well as the construction of Lie 2-groups which integrate $\mathfrak{s t r i n g}(n)$, see the papers of Baez-Crans-Schreiber-Stevenson [10], Henriques [31] and Schommer-Pries [47]. 


\subsubsection{The Heisenberg Lie 2-algebra}

As we mentioned earlier, central extensions of Lie algebras are classified by second cohomology. A famous example of this is the "Heisenberg Lie algebra", so named because it mimics the canonical commutation relations in quantum mechanics. Here we present a Lie 2-algebra generalization: the "Heisenberg Lie 2-algebra".

Consider the abelian Lie algebra of translations in position-momentum space:

$$
\mathbb{R}^{2}=\operatorname{span}(p, q) .
$$

Here, $p$ and $q$ are our names for the standard basis, the usual letters for momentum and position in physics. Up to rescaling, this Lie algebra has a single, nontrivial 2-cocycle:

$$
p^{*} \wedge q^{*} \in \Lambda^{2}\left(\mathbb{R}^{2}\right),
$$

where $p^{*}$ and $q^{*}$ comprise the dual basis. Thus it has a nontrivial central extension:

$$
0 \rightarrow \mathbb{R} \rightarrow \mathfrak{H} \rightarrow \mathbb{R}^{2} \rightarrow 0
$$

This central extension is called the Heisenberg Lie algebra. As a vector space, $\mathfrak{H}=\mathbb{R}^{3}$, and we call the basis vectors $p, q$ and $z$, where $z$ is central. When chosen with suitable normalization, they satisfy the relations:

$$
[p, q]=z, \quad[p, z]=0, \quad[q, z]=0 .
$$

These are the same as the canonical commutation relations in quantum mechanics, except that the generator $z$ would usually be a number, $-\mathrm{i} \hbar$. It is from this parallel that the Heisenberg Lie algebra derives its physical applications: a representation of $\mathfrak{H}$ is exactly a way of choosing linear operators $p, q$ and $z$ on a Hilbert space that satisfy the canonical commutation relations.

With Lie 2-algebras, we can repeat the process that yielded the Heisenberg Lie algebra to obtain a higher structure. Before we needed a 2-cocycle, but now we need a 3 -cocycle. Indeed, letting $p^{*}, q^{*}$ and $z^{*}$ be the dual basis of $\mathfrak{H}^{*}$, it is easy to check that $\gamma=p^{*} \wedge q^{*} \wedge z^{*}$ is a nontrivial 3-cocycle on $\mathfrak{H}$. Thus there is a Lie 2-algebra $\mathfrak{s t r i n g}_{\gamma}(\mathfrak{H})$, the Heisenberg Lie 2-algebra, which we denote by $\mathfrak{H e i s e n b e r g}$. Later, in Section 4, we will see how to integrate this Lie 2-algebra to a Lie 2-group. 
We suspect the Heisenberg Lie 2-algebra, like its Lie algebra cousin, is also important for physics. We also suspect that the pattern continues: the Heisenberg Lie 2-algebra may admit a "4-cocycle", and a central extension to a Lie 3-algebra. However, since we have not defined the cohomology of Lie $n$-algebras [43], we do not pursue this here.

\subsubsection{The supertranslation Lie 2-superalgebras}

This entire project began with the following puzzle: the classical superstring makes sense only in spacetimes of dimensions $n+2=3,4,6$ and 10, each of these numbers two higher than the dimensions of the normed division algebras. From the physics literature $[21,30]$, we see this is because a certain spinor identity holds in these spacetime dimensions and no others, namely the $\mathbf{3}-\boldsymbol{\psi}$ 's rule:

$$
(\psi \cdot \psi) \psi=0
$$

for all spinors $\psi \in S_{+}$. Here, the dot denotes an operation that takes two spinors and and outputs a vector, which in the above identity acts on the original spinor. In notation more typical of the physics literature, this would usually be written as:

$$
\left(\bar{\psi} \gamma^{\mu} \psi\right) \gamma_{\mu} \psi=0
$$

though it takes a number of different guises. For instance, one equivalent form is to say that all spinors square to null vectors in these dimensions:

$$
\|\psi \cdot \psi\|^{2}=0 .
$$

See Huerta [33], Section 2.4 for a full discussion.

We can understand this identity in terms of division algebras, as reviewed in the first paper of this series [5]. In some sense, this solves the puzzle we began with, but leaves us with another: what is the meaning of the $3-\psi$ 's rule itself? The answer, as we described in our second paper [6], lies in Lie algebra cohomology: it is a cocycle condition.

To understand this, we need to introduce supersymmetry. In any dimension, a symmetric bilinear intertwining operator that eats two spinors and spits out a vector gives rise to a "super-Minkowski spacetime" [20]. The infinitesimal translation symmetries of this object form a Lie superalgebra, which we call the "supertranslation algebra", $\mathcal{T}$. The cohomology of this Lie superalgebra is interesting and apparently rather subtle [14, 41,42]. We 
shall see that its third cohomology is nontrivial in dimensions $n+2=3,4$, 6 and 10 , thanks to the $3-\psi$ 's rule.

For arbitrary superspacetimes, the cohomology of $\mathcal{T}$ is not explicitly known. Techniques to compute it have been described by Brandt [14], who applied them in dimension 5 and below. Movshev et al. [41, 42] showed how to augment these techniques using computer algebra systems, such as LiE [19], and fully describe the cohomology in dimensions less than 11 in this way.

Based on the work of these authors, it seems likely that the 3rd cohomology of $\mathcal{T}$ is nontrivial in sufficiently large dimensions. We conjecture, however, that dimensions $n+2$ are the only ones with Lorentz-invariant 3 -cocycles. Exploratory calculations with LiE bare this conjecture out, but the general answer appears to be unknown.

Let us see how division algebras get into the game by using them to construct $\mathcal{T}$ in the relevant dimensions - 3, 4, 6 and 10. Recall that, by a classic result of Hurwitz [34], there are precisely four normed division algebras: the real numbers, $\mathbb{R}$, the complex numbers, $\mathbb{C}$, the quaternions, $\mathbb{H}$, and the octonions $\mathbb{O}$. These have dimensions $n=1,2,4$, and 8 , respectively.

Most properties of the division algebras are familiar from working with complex numbers. Each division algebra $\mathbb{K}$ is normed, in the sense that it is equipped with a norm $|\cdot|$ satisfying:

$$
|a b|=|a||b| .
$$

Each division algebra also has a conjugation, a linear map $*: \mathbb{K} \rightarrow \mathbb{K}$ satisfying:

$$
(a b)^{*}=b^{*} a^{*}, \quad a^{* *}=a .
$$

We can use this conjugation to write the norm:

$$
|a|^{2}=a a^{*}=a^{*} a .
$$

Not familiar from complex numbers, however, is nonassociativity. While $\mathbb{R}$, $\mathbb{C}$, and $\mathbb{H}$ are all associative, the octonions, $\mathbb{O}$, are not. However, they come close. They are alternative: every subalgebra generated by two elements is associative. Indeed, every normed division algebra is alternative.

We can now systematically use the normed division algebra $\mathbb{K}$ of dimension $n$ to construct the superstranlation algebra $\mathcal{T}$ for spacetime of dimension $n+2$. Most of this construction is well known $[2,18,35,40,52]$, although we 
learned it from Manogue and Schray $[49,48]$. First, the vector representation $V$ of $\operatorname{Spin}(n+1,1)$ is defined to be the set of $2 \times 2$ Hermitian matrices over $\mathbb{K}$ :

$$
V=\left\{\left(\begin{array}{cc}
t+x & y^{*} \\
y & t-x
\end{array}\right): t, x \in \mathbb{R}, y \in \mathbb{K}\right\}
$$

Note that this is a $(n+2)$-dimensional vector space. We define the Minkowski norm on this space using the determinant:

$$
-\operatorname{det}\left(\begin{array}{cc}
t+x & y^{*} \\
y & t-x
\end{array}\right)=-t^{2}+x^{2}+|y|^{2} .
$$

The minus sign ensures that we have signature $(n+1,1)$. Thus, the Lorentz group $\operatorname{Spin}(n+1,1)$, the double-cover of $\mathrm{SO}_{0}(n+1,1)$, acts on $V$ via determinant-preserving linear transformations.

While vectors are $2 \times 2$ matrices over $\mathbb{K}$, spinors are column vectors in $\mathbb{K}^{2}$. Indeed, the irreducible spinor representations of $\operatorname{Spin}(n+1,1)$ are both defined on $\mathbb{K}^{2}$ :

$$
S_{+}=\mathbb{K}^{2}, \quad S_{-}=\mathbb{K}^{2},
$$

but with slightly different actions of $\operatorname{Spin}(n+1,1)$. We shall avoid specifying these actions explicitly. For details, see Section 3 of our first paper [5], or Chapter 2 of Huerta [33].

Finally, for both $S_{+}$and $S_{-}$, there is a symmetric, bilinear, $\operatorname{Spin}(n+1,1)$ equivariant map:

$$
\cdot: S_{ \pm} \otimes S_{ \pm} \rightarrow V
$$

Despite our notation, we suggestively call this map the bracket of spinors. The form of this map is particularly charismatic on $S_{-}$, thanks to our use of $\mathbb{K}$ : just multiply the column vector $\psi$ by the row vector $\phi^{\dagger}$ and take the Hermitian part of the resulting $2 \times 2$ matrix:

$$
\psi \cdot \phi=\psi \phi^{\dagger}+\phi \psi^{\dagger}, \quad \psi, \phi \in S_{-} .
$$

Here, the dagger denotes the conjugate transpose, $\psi^{\dagger}=\left(\psi^{*}\right)^{T}$. On $S_{+}$, it is only slightly more complex, as our choice of action forces us to apply trace 
reversal - for a $2 \times 2$ matrix $A$, we define:

$$
\tilde{A}=A-\operatorname{tr}(A) .
$$

Then we can write:

$$
\psi \cdot \phi=\psi \widetilde{\phi^{\dagger}+\phi} \psi^{\dagger}, \quad \psi, \phi \in S_{+}
$$

In either case, the result is a $2 \times 2$ Hermitian matrix - a vector! The fact that these maps are $\operatorname{Spin}(n+1,1)$-equivariant is checked in our previous paper [5].

Finally, we fuse these two representations of $\operatorname{Spin}(n+1,1)$ together into a single object, the supertranslation algebra $\mathcal{T}$. This is a Lie superalgebra whose even part consists of vectors and odd part consists of spinors:

$$
\mathcal{T}_{0}=V, \quad \mathcal{T}_{1}=S_{+}
$$

The bracket on $\mathcal{T}$ is defined to vanish unless we bracket a spinor $\psi$ with a spinor $\phi$, in which case the bracket is simply $\psi \cdot \phi$. Since this operation is symmetric and spinors are odd, the bracket operation is gradedantisymmetric overall. Furthermore, the Jacobi identity holds trivially, thanks to the near triviality of the bracket. Thus $\mathcal{T}$ is indeed, a Lie superalgebra.

Moreover, $\mathcal{T}$ has a nontrivial 3-cocycle, thanks to the $3-\psi$ 's rule. We define a 3 -cochain, which eats a vector, $A$, and two spinors, $\psi, \phi$, and vanishes otherwise, as follows:

$$
\alpha(A, \psi, \phi)=g(A, \psi \cdot \phi)
$$

Here, $g$ is the Minkowski inner product on $V$.

Theorem 9. In dimensions 3, 4, 6 and 10, the supertranslation algebra $\mathcal{T}$ has a nontrivial, Lorentz-invariant even 3-cocycle $\alpha$ taking values in the trivial representation $\mathbb{R}$. Decomposing the graded exterior power $\Lambda^{3} \mathcal{T}$ into the direct sum $\Lambda^{3} \mathcal{T} \cong \bigoplus_{p+q=3} \Lambda^{p} V \otimes \operatorname{Sym}^{q} S_{+}$, we define the 3-cocycle:

$$
\alpha: \Lambda^{3} \mathcal{T} \rightarrow \mathbb{R}
$$

as the unique 3-cochain which takes the value:

$$
\alpha(A \wedge \psi \wedge \phi)=g(A, \psi \cdot \phi)
$$


"ATMP-16-5-A4-HUE" — 2013/4/30 — 17:32 — page 1520 — \#36

on the $V \otimes \mathrm{Sym}^{2} S_{+}$direct summand of $\Lambda^{3} \mathcal{T}$, and vanishes otherwise, for vectors $A \in V$ and spinors $\psi, \phi \in S_{+}$.

Proof. See the proof of Theorem 14 in our previous paper [6].

There is thus a Lie 2-superalgebra, the supertranslation Lie 2superalgebra, $\mathfrak{s t r i n g} \mathfrak{g}_{\alpha}(\mathcal{T})$. There is much more that one can do with the cocycle $\alpha$, however. We can use it to extend not just the supertranslations $\mathcal{T}$ to a Lie 2 -superalgebra, but the full Poincaré superalgebra, $\mathfrak{s o}(V) \ltimes \mathcal{T}$. We turn to this now.

\subsubsection{Superstring Lie 2-superalgebras}

One of the principal themes of theoretical physics over the last century has been the search for the underlying symmetries of nature. This began with special relativity, which could be summarized as the discovery that the laws of physics are invariant under the action of the Poincare group:

$$
\operatorname{ISO}(V)=\operatorname{Spin}(V) \ltimes V .
$$

Here, $V$ is the set of vectors in Minkowski spacetime and acts on Minkowski spacetime by translation, while $\operatorname{Spin}(V)$ is the Lorentz group: the double cover of $\mathrm{SO}_{0}(V)$, the connected component of the group of symmetries of the Minkowski norm. Much of the progress in physics since special relativity has been associated with the discovery of additional symmetries, like the $U(1) \times$ $\mathrm{SU}(2) \times \mathrm{SU}(3)$ symmetries of the Standard Model of particle physics [4].

Today, "supersymmetry" could be summarized as the hypothesis that the laws of physics are invariant under the "Poincaré supergroup", which is larger than the Poincaré group:

$$
\operatorname{SISO}(V)=\operatorname{Spin}(V) \ltimes T .
$$

Here, $V$ is again the set of vectors in Minkowski spacetime and $\operatorname{Spin}(V)$ is the Lorentz group, but $T$ is the supergroup of translations on Minkowski "superspacetime". Although we have not yet learned enough supergeometry to talk about $T$ precisely, we have already met its infinitesimal approximation in the last section: the superstranslation algebra, $\mathcal{T}=V \oplus S_{+}$. We think of the spinor representation $S_{+}$as giving extra, supersymmetric translations, or "supersymmetries".

In this paper, we show how to further extend the Poincaré supergroup to include higher symmetries, thanks to the normed division algebras. That 
is, we will show that in dimensions $n+2=3,4,6$ and 10 , one can extend the Poincaré supergroup $\operatorname{SISO}(n+1,1)$ to a "Lie 2 -supergroup" we call Superstring $(n+1,1)$.

We begin this construction in this section by working at the infinitesimal level. Using the 3-cocycle $\alpha$, we construct a Lie 2-superalgebra,

$$
\mathfrak{s u p e r s t r i n g}(n+1,1),
$$

which extends the Poincaré superalgebra in dimension $n+2$ :

$$
\mathfrak{s i s o}(n+1,1)=\mathfrak{s o}(n+1,1) \ltimes \mathcal{T}
$$

This is possible because $\alpha$ is invariant under the action of the Lorentz algebra, $\mathfrak{s o}(n+1,1)$. This is manifestly true, because $\alpha$ is built from equivariant maps.

As we shall see, this invariance implies that $\alpha$ is a cocycle, not merely on the supertranslation algebra, but on the full Poincaré superalgebra, $\mathfrak{s i s o}(n+$ $1,1)$. We can extend $\alpha$ to this larger algebra in a trivial way: define the unique extension which vanishes unless all of its arguments come from $\mathcal{T}$. Doing this, $\alpha$ remains a cocycle, even though the Lie bracket (and thus $d$ ) has changed. Moreover, it remains nontrivial. All of this is contained in the following proposition:

Proposition 10. Let $\mathfrak{g}$ and $\mathfrak{h}$ be Lie superalgebras such that $\mathfrak{g}$ acts on $\mathfrak{h}$, and let $R$ be a representation of $\mathfrak{g} \ltimes \mathfrak{h}$. Given any $R$-valued $n$-cochain $\omega$ on $\mathfrak{h}$, we can uniquely extend it to an $n$-cochain $\tilde{\omega}$ on $\mathfrak{g} \ltimes \mathfrak{h}$ that takes the value of $\omega$ on $\mathfrak{h}$ and vanishes on $\mathfrak{g}$. When $\omega$ is even, we have:

(1) $\tilde{\omega}$ is closed if and only if $\omega$ is closed and $\mathfrak{g}$-equivariant.

(2) $\tilde{\omega}$ is exact if and only if $\omega=d \theta$, for $\theta$ a $\mathfrak{g}$-equivariant $(n-1)$-cochain on $\mathfrak{h}$.

Proof. See the proof of Proposition 20 in our previous paper [6].

Thus we can extend $\alpha$ to a nontrivial cocycle on the Poincaré Lie superalgebra, simply by defining $\alpha$ to vanish outside of the supertranslation algebra. Thanks to Theorem 8 , we know that $\alpha$ lets us extend $\mathfrak{s i s o}(n+1,1)$ to a Lie 2-superalgebra:

Theorem 11. In dimensions 3, 4, 6 and 10, there exists a Lie 2-superalgebra formed by extending the Poincaré superalgebra $\mathfrak{s i s o}(n+1,1)$ by the 3-cocycle $\alpha$, which we call we the superstring Lie 2-superalgebra, $\mathfrak{s u p e r s t r i n g}(n+1,1)$. 
"ATMP-16-5-A4-HUE" — 2013/4/30 — 17:32 — page 1522 — \#38

\section{Integrating nilpotent Lie $n$-algebras}

Any mathematician worth her salt knows that we can easily construct Lie algebras as the infinitesimal versions of Lie groups, and that a more challenging inverse construction exists: we can "integrate" Lie algebras to get Lie groups. By analogy, we expect that the same is true of Lie $n$-algebras and Lie $n$-groups: that we can construct Lie $n$-algebras as the infinitesimal versions of Lie $n$-groups, and we can "integrate" Lie $n$-algebras to obtain Lie $n$-groups.

In fact, it is easy to see how to obtain slim Lie $n$-algebras from slim Lie $n$-groups. As we saw in Section 3 , slim Lie $n$-algebras are built from $(n+1)$-cocycles in Lie algebra cohomology. Remember, $p$-cochains on the Lie algebra $\mathfrak{g}$ are linear maps:

$$
C^{p}(\mathfrak{g}, \mathfrak{h})=\left\{\omega: \Lambda^{p} \mathfrak{g} \rightarrow \mathfrak{h}\right\},
$$

where $\mathfrak{h}$ is a representation of $\mathfrak{g}$, though we shall restrict ourselves to the trivial representation $\mathfrak{h}=\mathbb{R}$ in this section.

On the other hand, in Section 2, we saw that slim Lie $n$-groups are built from $(n+1)$-cocycles in Lie group cohomology, at least for $n=2$. Remember, $p$-cochains on $G$ are smooth maps:

$$
C^{p}(G, H)=\left\{f: G^{p} \rightarrow H\right\},
$$

where $H$ is an abelian group on which $G$ acts by automorphism, though we shall restrict ourselves to $H=\mathbb{R}$ with trivial action in this section.

Thus, to derive a Lie $n$-algebra from a Lie $n$-group, just differentiate the defining Lie group $(n+1)$-cocycle at the identity to obtain a Lie algebra $(n+1)$-cocycle. In other words, for every Lie group $G$ with Lie algebra $\mathfrak{g}$, there is a cochain map:

$$
D: C^{\bullet}(G) \rightarrow C^{\bullet}(\mathfrak{g}),
$$

given by differentiation. Here, we have omitted reference to the coefficients $H$ and $\mathfrak{h}$ because both are assumed to be $\mathbb{R}$. We continue this practice for the rest of the section.

Going the other way, however, is challenging - integrating a Lie $n$-algebra is harder, even when the Lie $n$-algebra in question is slim. Nonetheless, this challenge has been met. Building on the earlier work of Getzler [28] on integrating nilpotent Lie $n$-algebras, Henriques [31] has shown that any 
“ATMP-16-5-A4-HUE" — 2013/4/30 — 17:32 — page 1523 — \#39

Lie $n$-algebra can be integrated to a "Lie $n$-group", which Henriques defines as a sort of smooth Kan complex in the category of Banach manifolds. More recently, Schreiber [50] has generalized this integration procedure to a setting much more general than that of Banach manifolds, including both supermanifolds and manifolds with infinitesimals. For both Henriques and Schreiber, the definition of Lie $n$-group is weaker than the one we sketched in Section 2 - it weakens the notion of multiplication so that the product of two group "elements" is only defined up to equivalence. This level of generality seems essential for the construction to work for every Lie $n$-algebra.

However, for some Lie $n$-algebras, we can integrate them using the more naive idea of Lie $n$-group we prefer in this paper: a smooth $n$-category with one object in which every $k$-morphism is weakly invertible, for all $1 \leq k \leq n$. We shall see that, for some slim Lie $n$-algebras, we can integrate the defining Lie algebra $(n+1)$-cocycle to obtain a Lie group $(n+1)$-cocycle. In other words, for certain Lie groups $G$ with Lie algebra $\mathfrak{g}$, there is a cochain map:

$$
\int: C^{\bullet}(\mathfrak{g}) \rightarrow C^{\bullet}(G) .
$$

which is a chain homotopy inverse to differentiation.

When is this possible? We can always differentiate Lie group cochains to obtain Lie algebra cochains, but if we can also integrate Lie algebra cochains to obtain Lie group cochains, the cohomology of the Lie group and its Lie algebra will coincide:

$$
H^{\bullet}(\mathfrak{g}) \cong H^{\bullet}(G) .
$$

By a theorem of van Est [55], this happens when all the homology groups of $G$, as a topological space, vanish.

Thus, we should look to Lie groups with vanishing homology for our examples. How bad can things be when the Lie group is not homologically trivial? To get a sense for this, recall that any semisimple Lie group $G$ is diffeomorphic to the product of its maximal compact subgroup $K$ and a contractible space $C$ :

$$
G \approx K \times C .
$$

When $K$ is a point, $G$ is contractible, and certainly has vanishing homology. At the other extreme, when $C$ is a point, $G$ is compact. And indeed, in this case there is no hope of obtaining a nontrivial cochain map from Lie algebra cochains to Lie group cochains:

$$
\int: C^{\bullet}(\mathfrak{g}) \rightarrow C^{\bullet}(G)
$$

because every smooth cocycle on a compact group is trivial $[8,55]$. 
This fact provided an obstacle to early attempts to integrate Lie 2algebras. For instance, consider the string Lie 2-algebra $\mathfrak{s t r i n g}(n)$ we described in Section 3.1.1. Recall that it is the slim Lie 2-algebra $\mathfrak{s t r i n g} \mathfrak{g}_{j}(\mathfrak{s o}(n))$, where $j$ is the canonical 3 -cocycle on $\mathfrak{s o}(n)$, given by combining the Killing form with the bracket:

$$
j=\langle-,[-,-]\rangle .
$$

One could attempt to integrate $\mathfrak{s t r i n g}(n)$ to a slim Lie 2-group String $_{\int}(\mathrm{SO}(n))$, where $\int j$ is a Lie group 3-cocycle on $\mathrm{SO}(n)$ which somehow integrates $j$, but because the compact group $\mathrm{SO}(n)$ admits no nontrivial smooth Lie group cocycles, this idea fails.

The real lesson of the string Lie 2-algebra is that, once again, our notion of Lie 2-group is not general enough. By generalizing the concept of Lie 2-group, various authors, like Baez-Crans-Schreiber-Stevenson [10], Henriques [31] and Schommer-Pries [47], were successful in integrating $\mathfrak{s t r i n g}(n)$.

Nonetheless, there is a large class of Lie $n$-algebras for which our Lie $n$-groups are general enough. In particular, when $G$ is an "exponential" Lie group, the story is completely different. A Lie group or Lie algebra is called exponential if the exponential map

$$
\exp : \mathfrak{g} \rightarrow G
$$

is a diffeomorphism. For instance, all simply connected nilpotent Lie groups are exponential, although the reverse is not true. Certainly, all exponential Lie groups have vanishing homology, because $\mathfrak{g}$ is contractible. We caution the reader that some authors use the term "exponential" merely to indicate that the exponential map is surjective.

When $G$ is an exponential Lie group with Lie algebra $\mathfrak{g}$, we can use a geometric technique developed by Houard [32] to construct a cochain map:

$$
\int: C^{\bullet}(\mathfrak{g}) \rightarrow C^{\bullet}(G) .
$$

The basic idea behind this construction is simple, a natural outgrowth of a familiar concept from the cohomology of Lie algebras. Because a Lie algebra $p$-cochain is a linear map:

$$
\omega: \Lambda^{p} \mathfrak{g} \rightarrow \mathbb{R},
$$

using left translation, we can view $\omega$ as defining a $p$-form on the Lie group $G$. So, we can integrate this $p$-form over $p$-simplices in $G$. Thus we can 
define a smooth function:

$$
\int \omega: G^{p} \rightarrow \mathbb{R}
$$

by viewing the integral of $\omega$ as a function of the vertices of a $p$-simplex:

$$
\int \omega\left(g_{1}, g_{2}, \ldots, g_{p}\right)=\int_{\left[1, g_{1}, g_{1} g_{2}, \ldots, g_{1} g_{2} \cdots g_{p}\right]} \omega .
$$

For the right-hand side to truly be a function of the $p$-tuple $\left(g_{1}, g_{2}, \ldots, g_{p}\right)$, we will need a standard way to "fill out" the $p$-simplex $\left[1, g_{1}, g_{1} g_{2}, \ldots\right.$, $\left.g_{1} g_{2} \cdots g_{p}\right]$, based only on its vertices. It is here that the fact that $G$ is exponential is key: in an exponential group, we can use the exponential map to define a unique path from the identity 1 to any group element. We think of this path as giving a 1-simplex, $[1, g]$, and we can extend this idea to higher-dimensional $p$-simplices.

Therefore, when $G$ is exponential, we can construct $\int$. Using this cochain map, it is possible to integrate the slim Lie $n$-algebra $\mathfrak{b r a n e} \mathfrak{e}_{\omega}(\mathfrak{g})$ to the slim Lie $n$-group Brane ${ }_{\omega} \omega(G)$.

We proceed as follows. In Section 4.1, we construct $\int$ and show that, along with $D$, it gives a homotopy equivalence between the complexes $C^{\bullet}(\mathfrak{g})$ and $C^{\bullet}(G)$. In Section 4.2, we use $\int$ to integrate the Heisenberg Lie 2-algebra of Section 3.1.2. Later, in Section 7, we shall see that this construction can be "superized", and integrate Lie $n$-superalgebras to $n$-supergroups. Finally, so that the reader can see concrete calculations with $\int$, in Appendix A we work out explicit formulas for the Lie group $p$-cochains one obtains from integrating Lie algebra $p$-cochains, for $p=0,1,2$ and 3 .

\subsection{Integrating Lie algebra cochains}

In what follows, we shall see that for an exponential Lie group $G$, we can construct simplices in $G$ that get along with the action of $G$ on itself. Since we can treat any $p$-cochain $\omega$ on $\mathfrak{g}$ as a left-invariant $p$-form on $G$, we can integrate $\omega$ over a $p$-simplex in $S$ in $G$. Regarding $\int_{S} \omega$ as a function of the vertices of $S$, we will see that it defines a Lie group $p$-cochain. The fact that this is a cochain map is purely geometric: it follows automatically from Stokes' theorem.

Let us begin by replacing the cohomology of $\mathfrak{g}$ with the cohomology of left-invariant differential forms on $G$. Recall that the cohomology of the Lie 
"ATMP-16-5-A4-HUE" — 2013/4/30 — 17:32 — page 1526 — \#42

algebra $\mathfrak{g}$ is given by the Lie algebra cochain complex, $C^{\bullet}(\mathfrak{g})$, which at level $p$ consists of $p$-linear maps from $\mathfrak{g}$ to $\mathbb{R}$ :

$$
C^{p}(\mathfrak{g})=\left\{\omega: \Lambda^{p} \mathfrak{g} \rightarrow \mathbb{R}\right\} .
$$

We already defined this for Lie superalgebras in Section 3. In that section, we saw that the coboundary map $d$ on this complex is usually defined by a rather lengthy formula, but here we shall substitute an equivalent, more geometric definition. Since we can think of the Lie algebra $\mathfrak{g}$ as the tangent space $T_{1} G$, we can think of a $p$-cochain on $\mathfrak{g}$ as giving a $p$-form on this tangent space. Using left translation on the group, we can translate this $p$-form over $G$ to define a $p$-form on all of $G$. This $p$-form is left-invariant, and it is easy to see that any left-invariant $p$-form on $G$ arises in this way.

So, in fact, we could just as well define

$$
C^{p}(\mathfrak{g})=\{\text { left-invariant } p \text {-forms on } G\} .
$$

It is well-known that the de Rham differential of a left-invariant $p$-form $\omega$ is again left-invariant, and remarkably, the formula for $d \omega_{1}$ involves only the Lie bracket on $\mathfrak{g}$. This formula is Chevalley and Eilenberg's original definition of $d$ [17], the one we gave in Section 3, albeit adapted for Lie superalgebras. In this section, we may forget about this messy formula, and use the de Rham differential instead.

The cohomology of the Lie group $G$ is given by the Lie group cochain complex, $C^{\bullet}(G)$, which at level $p$ is given by the set of smooth functions from $G^{p}$ to $\mathbb{R}$ :

$$
C^{p}(G)=\left\{f: G^{p} \rightarrow \mathbb{R}\right\} .
$$

We have already discussed this in Section 2. The coboundary map $d$ on this complex is usually defined by a complicated formula we gave in that section, but we can give it a more geometric description just as we did in the case of Lie algebras.

Since we are going to construct a cochain map by integrating $p$-forms over $p$-simplices, it would be best to view Lie group cohomology in terms of simplices now. To this end, let us define a combinatorial $\boldsymbol{p}$-simplex in the group $G$ to be an $(p+1)$-tuple of elements of $G$, which we call the vertices in this context. Of course, $G$ acts on the set of combinatorial $p$-simplices by left multiplication of the vertices.

Now, we would like to think of Lie group $p$-cochains as "smooth, homogeneous, $\mathbb{R}$-valued cochains" on the free abelian group on combinatorial 
$p$-simplices. Of course, we need to say what this means. We say an $\mathbb{R}$ valued $p$-cochain $F$ is homogeneous if it is invariant under the left action of $G$, and that it is smooth if the corresponding map

$$
F: G^{p+1} \rightarrow \mathbb{R}
$$

is smooth. Now if

$$
C_{H}^{p}(G)=\{\text { smooth homogeneous } p \text {-cochains }\}
$$

denotes the abelian group of all smooth, homogeneous $p$-cochains, there is a standard way to make $C_{H}^{\bullet}(G)$ into a cochain complex. Just take the coboundary operator to be:

$$
d F=F \circ \partial,
$$

where $\partial$ is the usual boundary operator on $p$-chains. It is automatic that $d^{2}=0$.

In fact, this cochain complex is isomorphic to the original one, which we distinguish as the inhomogeneous cochains:

$$
C_{I}^{p}(G)=\left\{f: G^{p} \rightarrow \mathbb{R}\right\}
$$

To see this, note that any inhomogeneous cochain:

$$
f: G^{p} \rightarrow \mathbb{R}
$$

gives rise to a unique, smooth, homogeneous $p$-cochain $F$, by defining:

$$
F\left(g_{0}, \ldots, g_{p}\right)=f\left(g_{0}^{-1} g_{1}, g_{1}^{-1} g_{2}, \ldots, g_{p-1}^{-1} g_{p}\right)
$$

for each combinatorial $p$-simplex $\left(g_{0}, \ldots, g_{p}\right)$. Conversely, every smooth, homogeneous $p$-cochain $F$ gives a unique inhomogeneous $p$-cochain $f: G^{p} \rightarrow$ $\mathbb{R}$, by defining:

$$
f\left(g_{1}, \ldots, g_{p}\right)=F\left(1, g_{1}, g_{1} g_{2}, \ldots, g_{1} g_{2} \ldots g_{p}\right) .
$$

Finally, note that these isomorphisms commute with the coboundary operators on $C_{H}^{\bullet}(G)$ and $C_{I}^{\bullet}(G)$. Henceforth, we will write $C^{\bullet}(G)$ to mean either complex. 
These simplicial notions will permit us to define a cochain map from the Lie algebra complex to the Lie group complex:

$$
C^{\bullet}(\mathfrak{g}) \rightarrow C^{\bullet}(G)
$$

For $\omega \in C^{p}(\mathfrak{g})$, the idea is to define an element $\int \omega \in C^{p}(G)$ by integrating the left-invariant $p$-form $\omega$ over a $p$-simplex $S$ in the group $G$. In other words, the value which $\int \omega$ assigns to $S$ is defined to be:

$$
\left(\int \omega\right)(S)=\int_{S} \omega
$$

This is nice because Stokes' theorem will tell us it is a cochain map:

$$
\left(\int d \omega\right)(S)=\int_{S} d \omega=\int_{\partial S} \omega=d\left(\int \omega\right)(S)
$$

The only hard part is defining $p$-simplices in $G$ in such a way that $\int \omega$ is actually a smooth, homogeneous $p$-cochain. It is here that the fact that $G$ is exponential is key.

Note that, up until this point, we have only discussed combinatorial $p$-simplices, which have no relationship to the Lie group structure of $G$ they are just $(p+1)$-tuples of vertices. We now wish to "fill out" the combinatorial simplices. That is, we want to create a rule that to any $(p+1)$-tuple $\left(g_{0}, \ldots, g_{p}\right)$ of vertices in $G$ assigns a filled $p$-simplex in $G$, which we denote

$$
\left[g_{0}, \ldots, g_{p}\right] \text {. }
$$

In order to prove that $\int \omega$ is smooth, we need smoothness conditions for this rule, and in order to prove $\int \omega$ is homogeneous, we shall require the lefttranslate of a $p$-simplex to again be a $p$-simplex. In other words, we need:

$$
g\left[g_{0}, \ldots, g_{p}\right]=\left[g g_{0}, \ldots, g g_{p}\right]
$$

We make this precise as follows.

Definition 12. Let $\Delta^{p}$ denote $\left\{\left(x_{0}, \ldots, x_{p}\right) \in \mathbb{R}^{p+1}: \sum x_{i}=1, x_{i} \geq 0\right\}$, the standard $p$-simplex in $\mathbb{R}^{p+1}$. Given a collection of smooth maps

$$
\varphi_{p}: \Delta^{p} \times G^{p+1} \rightarrow G,
$$

for each $p \geq 0$, we say this collection defines a left-invariant notion of simplices in $G$ if it satisfies: 
(1) The vertex property. For any $(p+1)$-tuple, the restriction

$$
\varphi_{p}: \Delta^{p} \times\left\{\left(g_{0}, \ldots, g_{p}\right)\right\} \rightarrow G
$$

sends the vertices of $\Delta^{p}$ to $g_{0}, \ldots, g_{p}$, in that order. We denote this restriction by

$$
\left[g_{0}, \ldots, g_{p}\right] \text {. }
$$

We call this map a $\boldsymbol{p}$-simplex, and regard it as a map from $\Delta^{p}$ to $G$.

(2) Left-invariance. For any $p$-simplex $\left[g_{0}, \ldots, g_{p}\right]$ and any $g \in G$, we have:

$$
g\left[g_{0}, \ldots, g_{p}\right]=\left[g g_{0}, \ldots, g g_{p}\right] .
$$

(3) The face property. For any $p$-simplex

$$
\left[g_{0}, \ldots, g_{p}\right]: \Delta^{p} \rightarrow G
$$

the restriction to a face of $\Delta^{p}$ is a $(p-1)$-simplex.

Note that the second condition just says that the map

$$
\varphi_{p}: \Delta^{p} \times G^{p+1} \rightarrow G
$$

is equivariant with respect to the left action of $G$, where we take $G$ to act trivially on $\Delta^{p}$.

On any group equipped with a left-invariant notion of simplex, we have the following result:

Proposition 13. Let $G$ be a Lie group equipped with a left-invariant notion of simplices, and let $\mathfrak{g}$ be its Lie algebra. Then there is a cochain map from the Lie algebra cochain complex to the Lie group cochain complex

$$
\int: C^{\bullet}(\mathfrak{g}) \rightarrow C^{\bullet}(G)
$$

given by integration - that is, if $\omega$ is a left-invariant $p$-form on $G$, and $S$ is a p-simplex in $G$, then define:

$$
\left(\int \omega\right)(S)=\int_{S} \omega .
$$


"ATMP-16-5-A4-HUE" — 2013/4/30 — 17:32 — page 1530 — \#46

Proof. Let $\omega \in C^{p}(\mathfrak{g})$. We have already noted that Stokes' theorem

$$
\int_{S} d \omega=\int_{\partial S} \omega
$$

implies that this map is a cochain map. We only need to check that $\int \omega$ really lands in $C^{p}(G)$. That is, that it is smooth and homogeneous. Because $G$ acts trivially on the coefficient group $\mathbb{R}$, homogeneity means that $\left(\int \omega\right)(S)$ is invariant of the left action of $G$ on $S$.

Indeed, note that we can pull the smooth, left-invariant $p$-form $\omega$ back along

$$
\varphi_{p}: \Delta^{p} \times G^{p+1} \rightarrow G .
$$

The result, $\varphi_{p}^{*} \omega$, is a smooth $p$-form on $\Delta^{p} \times G^{p+1}$, still invariant under the action of $G$. Integrating out the dependence on $\Delta^{p}$, we see this results in a smooth, invariant map:

$$
\int \omega: G^{p+1} \rightarrow \mathbb{R}
$$

which is precisely what we wanted to prove.

We would now like to show that any exponential Lie group $G$ comes with a left-invariant notion of simplices. Our essential tool for this is our ability to use the exponential map to connect any element of $G$ to the identity by a uniquely-defined path. If $h=\exp (X) \in G$ is such an element, we can then define the "based" 1 -simplex $[1, h]$ to be swept out by the path $\exp (t X)$, left translate this to define the general 1-simplex $[g, g h]$ as that swept out by the path $g \exp (t X)$, and proceed to define higher-dimensional simplices with the help of the exponential map and induction, using what we call the apex-base construction: given a definition of $(p-1)$-simplex, we define the $p$-simplex

$$
\left[1, g_{1}, \ldots, g_{p}\right]
$$

by using the exponential map to sweep out a path from 1 , the apex, to each point of the already defined $(p-1)$-simplex, the base:

$$
\left[g_{1}, \ldots, g_{p}\right] \text {. }
$$

Having done this, we can then use left translation to define the general -simplex:

$$
\left[g_{0}, g_{1}, \ldots, g_{p}\right]=g_{0}\left[1, g_{0}^{-1} g_{1}, \ldots, g_{0}^{-1} g_{p}\right]
$$


In fact, this construction also covers the 1-simplex case. All we need to kick off our induction is to define 0 -simplices to be points in $G$.

To make all this precise, we must use it to define smooth maps

$$
\varphi_{p}: \Delta^{p} \times G^{p+1} \rightarrow G
$$

for each $p \geq 0$. To overcome some analytic technicalities in constructing $\varphi_{p}$, we will also need to fix a smooth increasing function:

$$
\ell:[0,1] \rightarrow[0,1]
$$

which is 0 on a neighborhood of 0 , and then monotonically increases to 1 at 1 . We shall call $\ell$ the smoothing factor. We shall see latter that our choice of smoothing factor is immaterial: $\varphi_{p}$ depends on $\ell$, but integrals over simplices do not.

Let us begin by defining 0 -simplices as points. That is, we define

$$
\varphi_{0}: \Delta^{0} \times G \rightarrow G
$$

as the obvious projection.

Now, assume that we have defined $(p-1)$-simplices, so we have:

$$
\varphi_{p-1}: \Delta^{p-1} \times G^{p} \rightarrow G .
$$

Using this, we wish to define:

$$
\varphi_{p}: \Delta^{p} \times G^{p+1} \rightarrow G .
$$

But since we want this to be $G$-equivariant, we might as well define it for based $\boldsymbol{p}$-simplices: a simplex whose first vertex is 1 . So first, we will give a map:

$$
\tilde{f}_{p}: \Delta^{p} \times G^{p} \rightarrow G
$$

which we think of as giving us the based $p$-simplex

$$
\left[1, g_{1}, \ldots, g_{p}\right]
$$

for any $p$-tuple. We do this using the apex-base construction. First, the map $\varphi_{p-1}: \Delta^{p-1} \times G^{p} \rightarrow G$ can be extended to a map

$$
f_{p}:[0,1] \times \Delta^{p-1} \times G^{p} \rightarrow G
$$


by defining $f_{p}$ to be $\varphi_{p-1}$ on $\{1\} \times \Delta^{p-1} \times G^{p}$, to be 1 on $\{0\} \times \Delta^{p-1} \times G^{p}$, and using the exponential map to interpolate in between. Since $[0,1] \times \Delta^{p}$ is a kind of generalized prism, we take the liberty of calling $\{0\} \times \Delta^{p}$ the $\mathbf{0}$ face, and $\{1\} \times \Delta^{p}$ the $\mathbf{1}$ face.

Here, the requirement for smoothness complicates things slightly, because we shall actually need $f_{p}$ to be 1 on a neighborhood of the 0 face. So, to be precise, for $\left(t, x, g_{1}, \ldots, g_{p}\right) \in[0,1] \times \Delta^{p-1} \times G^{p}$, we have that $\varphi_{p-1}(x$, $\left.g_{0}, \ldots, g_{p}\right)$ is a point of $G$, say $\exp (X)$. Define:

$$
f_{p}\left(t, x, g_{0}, \ldots, g_{p}\right)=\exp (\ell(t) X) .
$$

where $\ell$ is the smoothing factor we mention above: a smooth increasing function which is 0 on a neighborhood of 0 , and then monotonically increases to 1 at 1 . This guarantees $f_{p}$ will be 1 on a neighborhood of the 0 face, and will match $\varphi_{p-1}$ on the 1 face.

Since $f_{p}$ is smooth and is constant on a neighborhood of the 0 face of the prism, $[0,1] \times \Delta^{p-1}$, we can quotient by this face and obtain a smooth map:

$$
\tilde{f}_{p}: \Delta^{p} \times G^{p} \rightarrow G .
$$

For definiteness, we can use the smooth quotient map defined by:

$$
\begin{array}{ccc}
q_{p}: \quad[0,1] \times \Delta^{p-1} & \rightarrow & \Delta^{p} \\
(t, x) & \mapsto & (1-t, t x)
\end{array}
$$

which we note sends the 0 face to the 0 th vertex of $\Delta^{p}$, and sends the vertices of $\Delta^{p-1}$ to the remaining vertices of $\Delta^{p}$, in order. Finally, to define the nonbased $p$-simplices, we extend by the left action of $G$-for any $g \in G$ and any $\left(x, g_{1}, \ldots, g_{p}\right) \in \Delta^{p} \times G^{p}$, set:

$$
\varphi_{p}\left(x, g, g g_{1}, \ldots, g g_{p}\right)=g \tilde{f}_{p}\left(x, g_{1}, \ldots, g_{p}\right) .
$$

This defines

$$
\varphi_{p}: \Delta^{p} \times G^{p+1} \rightarrow G .
$$

It just remains to check that:

Proposition 14. This defines a left-invariant notion of simplices on $G$, which we call the standard left-invariant notion of simplices with smoothing factor $\ell$. 
Proof. By construction, the $\varphi_{p}$ are all smooth and $G$-equivariant, so we only need to check the vertex property and the face property. We do this inductively.

For 0 -simplices, the vertex property is trivial. Assume it holds for $(p-1)$ simplices. In particular, the map

$$
\left[g_{1}, \ldots, g_{p}\right]: \Delta^{p-1} \rightarrow G
$$

sends the vertices of $\Delta^{p-1}$ to $g_{1}, \ldots, g_{p}$, in that order. By construction, the based $p$-simplex

$$
\left[1, g_{1}, \ldots, g_{p}\right]: \Delta^{p} \rightarrow G
$$

sends the 0 th vertex to 1 and the rest of the vertices to $g_{1}, \ldots, g_{p}$, since the $(p-1)$-simplex $\left[g_{1}, \ldots, g_{p}\right]$ has the vertex property and is defined to be the base of this $p$-simplex in the apex-base construction. By $G$-equivariance, this extends to all $p$-simplices.

For 0-simplices, the face property holds vacuously, and for 1-simplices it is the same as the vertex property. Now take $p \geq 2$, and assume the face property holds for all $k$-simplices with $k<p$. By $G$-equivariance, the face property will hold for all $p$-simplices as long as it holds for all based $p$-simplices, for instance:

$$
\left[1, g_{1}, \ldots, g_{p}\right]
$$

By the apex-base construction, the $(p-1)$-simplex $\left[g_{1}, \ldots, g_{p}\right]$ is the 0 th face of $\left[1, g_{1}, \ldots, g_{p}\right]$, since it was chosen as the base. For any other face, say the $i$ th face, the apex-base construction gives the $(p-1)$-simplex

$$
\left[1, g_{1}, \ldots, \hat{g}_{i}, \ldots, g_{p}\right]: \Delta^{p-1} \rightarrow G
$$

with 1 as apex, and the $(p-2)$-simplex $\left[g_{1}, \ldots, \hat{g}_{i}, \ldots, g_{p}\right]$ as base. Thus, the face property holds for the $p$-simplex $\left[1, g_{1}, \ldots, g_{p}\right]$.

While the existence of any left-invariant notion of simplices in $G$ suffices to integrate Lie algebra cochains, we have found an almost overwhelming wealth of these notions - one for each smoothing factor $\ell$. In fact, for 
the moment we will indicate the dependence of the standard notion of leftinvariant simplices on $\ell$ with a superscript:

$$
\varphi_{p}^{\ell}: \Delta^{p} \times G^{p+1} \rightarrow G .
$$

Of course, the dependence of $\varphi_{p}^{\ell}$ on $\ell$ passes to the individual simplices, so we give them a superscript as well:

$$
\left[g_{0}, \ldots, g_{p}\right]^{\ell}: \Delta^{p} \rightarrow G .
$$

Fortunately, however, the cochain map:

$$
\int: C^{\bullet}(\mathfrak{g}) \rightarrow C^{\bullet}(G)
$$

is independent of $\ell$. That is, if $\ell^{\prime}$ is another smoothing factor, we have:

$$
\int_{\left[g_{0}, \ldots, g_{p}\right]^{\ell}} \omega=\int_{\left[g_{0}, \ldots, g_{p}\right]^{\ell^{\prime}}} \omega
$$

for any left-invariant $p$-form $\omega$.

We shall prove this not by comparing the integrals for two smoothing factors, but rather computing the integral in a way that is manifestly independent of smoothing factor. We do this by showing that the role of the smoothing factor is basically to allow us to smoothly quotient the $p$-dimensional cube $[0,1]^{p}$ down to the standard $p$-simplex $\Delta^{p}$. Had we parameterized our $p$-simplices with cubes to begin with, we would have had no need for a smoothing factor. As a trade off, however, our proof that integration gives a cochain map would have required more care when analyzing the boundary.

Now we get to work. Rather than parameterizing the $p$-simplex on the domain $\Delta^{p}$ :

$$
\left[g_{0}, g_{1}, \ldots, g_{p}\right]^{\ell}: \Delta^{p} \rightarrow G
$$

we shall show how to parameterize it on the $p$-dimensional cube:

$$
\left\langle g_{0}, g_{1}, \ldots, g_{p}\right\rangle:[0,1]^{p} \rightarrow G,
$$

That is, these two functions have the same images - a $p$-simplex in $G$ with vertices $g_{0}, \ldots, g_{p} \in G$, they induce the same orientations on their images, and both traverse the image precisely once. So, as we shall prove, the integral over either simplex is the same. However, as we shall also see, the latter parameterization does not depend on the smoothing factor $\ell$. 
How do we discover the parameterization $\left\langle g_{0}, \ldots, g_{p}\right\rangle$ ? We just repeat the apex-base construction, but we avoid quotienting to down to $\Delta^{p}$ ! Begin by defining the 0 -simplices to map the 0 -dimensional cube to the indicated vertex:

$$
\left\langle g_{0}\right\rangle:\{0\} \rightarrow G
$$

Define a 1-simplex by using the exponential map to sweep out a path from $g_{0}$ to $g_{1}$ :

$$
\left\langle g_{0}, g_{1}\right\rangle:[0,1] \rightarrow G
$$

by defining:

$$
\left\langle g_{0}, g_{1}\right\rangle\left(t_{1}\right)=g_{0} \exp \left(t_{1} X_{1}\right), \quad t_{1} \in[0,1] .
$$

where $g_{0}^{-1} g_{1}=\exp \left(X_{1}\right)$. Now, define a 2 -simplex using the exponential map to sweep out paths from $g_{0}$ to the 1-simplex $\left\langle g_{1}, g_{2}\right\rangle$. That is, define:

$$
\left\langle g_{0}, g_{1}, g_{2}\right\rangle:[0,1]^{2} \rightarrow G
$$

to be given by:

$$
\left\langle g_{0}, g_{1}, g_{2}\right\rangle\left(t_{1}, t_{2}\right)=g_{0} \exp \left(t_{1} Z\left(X_{1}, t_{2} X_{2}\right)\right)
$$

where $g_{0}^{-1} g_{1}=\exp \left(X_{1}\right), g_{1}^{-1} g_{2}=\exp \left(X_{2}\right)$, and $Z$ denotes the BakerCampbell-Hausdorff series:

$$
g_{1} g_{2}=\exp \left(Z\left(X_{1}, X_{2}\right)\right)=\exp \left(X_{1}+X_{2}+\frac{1}{2}\left[X_{1}, X_{2}\right]+\cdots\right)
$$

Continuing in this manner, with a bit of work one can see that the $p$-simplex:

$$
\left\langle g_{0}, g_{1}, g_{2}, \ldots, g_{p-1}, g_{p}\right\rangle:[0,1]^{p} \rightarrow G
$$

is given by the horrendous formula:

$$
\begin{aligned}
& \left\langle g_{0}, \ldots, g_{p}\right\rangle\left(t_{1}, \ldots, t_{p}\right) \\
& \quad=g_{0} \exp \left(t_{1} Z\left(X_{1}, t_{2} Z\left(X_{2}, \ldots, t_{p-1} Z\left(X_{p-1}, t_{p} X_{p}\right) \ldots\right)\right)\right),
\end{aligned}
$$


where $g_{0}^{-1} g_{1}=\exp \left(X_{1}\right), g_{1}^{-1} g_{2}=\exp \left(X_{2}\right), \ldots, g_{p-1}^{-1} g_{p}=\exp \left(X_{p}\right)$. While horrendous, this formula is at least independent of the smoothing factor $\ell$, and this forms the basis of the following proposition:

Proposition 15. Let $G$ be an exponential Lie group with Lie algebra $\mathfrak{g}$, let $\ell$ be a smoothing factor, and equip $G$ with the standard left-invariant notion of simplices with smoothing factor $\ell$. For any p-simplex

$$
\left[g_{0}, \ldots, g_{p}\right]^{\ell}: \Delta^{p} \rightarrow G
$$

depending on $\ell$ and parameterized on the domain $\Delta^{p}$, there is a p-simplex:

$$
\left\langle g_{0}, \ldots, g_{p}\right\rangle:[0,1]^{p} \rightarrow G
$$

given by the formula:

$$
\begin{aligned}
& \left\langle g_{0}, \ldots, g_{p}\right\rangle\left(t_{1}, \ldots, t_{p}\right) \\
& \quad=g_{0} \exp \left(t_{1} Z\left(X_{1}, t_{2} Z\left(X_{2}, \ldots, t_{p-1} Z\left(X_{p-1}, t_{p} X_{p}\right) \ldots\right)\right)\right)
\end{aligned}
$$

where $g_{0}^{-1} g_{1}=\exp \left(X_{1}\right), g_{1}^{-1} g_{2}=\exp \left(X_{2}\right), \ldots, g_{p-1}^{-1} g_{p}=\exp \left(X_{p}\right)$. Then $\left\langle g_{0}, \ldots, g_{p}\right\rangle$ is independent of $\ell$, parameterized on the domain $[0,1]^{p}$, and has the same image and orientation as $\left[g_{0}, \ldots, g_{p}\right]^{\ell}$. Furthermore, for any p-form $\omega$ on $G$, the integral of $\omega$ is the same over either simplex:

$$
\int_{\left[g_{0}, \ldots, g_{p}\right]^{\ell}} \omega=\int_{\left\langle g_{0}, \ldots, g_{p}\right\rangle} \omega
$$

Sketch of proof. Equality of images and orientations follows from the apexbase construction, and equality of the integrals follows from reparameterization invariance - specifically the change of variables formula for multiple integrals, for which the monotonicity of $\ell$ becomes crucial.

Corollary 16. Let $G$ be an exponential Lie group with Lie algebra $\mathfrak{g}$, let $\ell$ be a smoothing factor, and equip $G$ with the standard left-invariant notion of simplices with smoothing factor $\ell$. Let

$$
\int: C^{\bullet}(\mathfrak{g}) \rightarrow C^{\bullet}(G)
$$

be the cochain map from Lie algebra cochains to Lie group cochains given by integration over simplices. Then the cochain map $\int$ is independent of the smoothing factor $\ell$. 
Proof. Recall that if $\omega$ is a left-invariant $p$-form on $G$, and $\left[g_{0}, \ldots, g_{p}\right]^{\ell}$ is a $p$-simplex in $G$, the cochain map $\int$ is defined by:

$$
\left(\int \omega\right)\left(g_{0}, \ldots, g_{p}\right)=\int_{\left[g_{0}, \ldots, g_{p}\right]^{\ell}} \omega
$$

By the previous proposition, this integral is equal to

$$
\int_{\left\langle g_{0}, \ldots, g_{p}\right\rangle} \omega
$$

where $\left\langle g_{0}, \ldots, g_{p}\right\rangle:[0,1]^{p} \rightarrow G$ is given as above, and is independent of $\ell$. Thus, $\int$ is also independent of $\ell$.

Having proven that the cochain map $\int$ is independent of smoothing factor, we will now allow the smoothing factor to recede into the background. Henceforth, we abuse terminology somewhat and speak of the standard leftinvariant notion of simplices to mean the standard notion with some implicit choice of smoothing factor.

The hard work of integrating Lie algebra cochains is now done. We would now like to go the other way, and show how to get a Lie algebra cochain from a Lie group cochain. This direction is much easier: in essence, we differentiate the Lie group cochain at the identity, and antisymmetrize the result. To do this, we make use of the fact that any element of the Lie algebra can be viewed as a directional derivative at the identity. The following result, due to van Est (c.f. [55], Formula 46) just says this map defines a cochain map:

Proposition 17. Let $G$ be a Lie group with Lie algebra $\mathfrak{g}$. Then there is a cochain map from the Lie group cochain complex to the Lie algebra cochain complex:

$$
D: C^{\bullet}(G) \rightarrow C^{\bullet}(\mathfrak{g})
$$

given by differentiation - that is, if $F$ is a homogeneous p-cochain on $G$, and $X_{1}, \ldots, X_{p} \in \mathfrak{g}$, then we can define:

$D F\left(X_{1}, \ldots, X_{p}\right)=\frac{1}{p !} \sum_{\sigma \in S_{p}} \operatorname{sgn}(\sigma) X_{\sigma(1)}^{1} \ldots X_{\sigma(p)}^{p} F\left(1, g_{1}, g_{1} g_{2}, \ldots, g_{1} g_{2} \ldots g_{p}\right)$,

where by $X_{i}^{j}$ we indicate that the operator $X_{i}$ differentiates only the $j$ th variable, $g_{j}$. 
"ATMP-16-5-A4-HUE" — 2013/4/30 — 17:32 — page 1538 — \#54

1538

JOHN HUERTA

Proof. See Houard [32], p. 224, Lemma 1.

Having now defined cochain maps

$$
\int: C^{\bullet}(\mathfrak{g}) \rightarrow C^{\bullet}(G)
$$

and

$$
D: C^{\bullet}(G) \rightarrow C^{\bullet}(\mathfrak{g})
$$

the obvious next question is whether or not this defines a homotopy equivalence of cochain complexes. Indeed, as proved by Houard, they do:

Theorem 18. Let $G$ be a Lie group equipped with a left-invariant notion of simplices, and $\mathfrak{g}$ its Lie algebra. The cochain map

$$
D \int: C^{\bullet}(\mathfrak{g}) \rightarrow C^{\bullet}(\mathfrak{g}),
$$

is the identity, whereas the cochain map

$$
\int D: C^{\bullet}(G) \rightarrow C^{\bullet}(G)
$$

is cochain-homotopic to the identity. Therefore the Lie algebra cochain complex $C^{\bullet}(\mathfrak{g})$ and the Lie group cochain complex $C^{\bullet}(G)$ are homotopy equivalent and thus have isomorphic cohomology.

Proof. See Houard [32], p. 234, Proposition 2.

\subsection{The Heisenberg Lie 2-group}

In Section 3.1.2, we met the Heisenberg Lie algebra, $\mathfrak{H}=\operatorname{span}(p, q, z)$. This is the three-dimensional Lie algebra where the generators $p, q$ and $z$ satisfy relations which mimic the canonical commutation relations from quantum mechanics:

$$
[p, q]=z, \quad[p, z]=0, \quad[q, z]=0 .
$$

As one can see from the above relations, $\mathfrak{H}$ is 2-step nilpotent: brackets of brackets are zero. 
We then met the Lie 2-algebra generalization, the Heisenberg Lie 2-algebra:

$$
\mathfrak{H e i s e n b e r g}=\mathfrak{s t r i n g} \mathfrak{g}_{\gamma}(\mathfrak{H}),
$$

built by extending $\mathfrak{H}$ with the 3 -cocycle $\gamma=p^{*} \wedge q^{*} \wedge z^{*}$, where $p^{*}, q^{*}$, and $z^{*}$ is the basis dual to $p, q$ and $z$.

It is easy to construct a Lie group $H$ with Lie algebra $\mathfrak{H}$. Just take the group of $3 \times 3$ upper triangular matrices with units down the diagonal:

$$
H=\left\{\left(\begin{array}{ccc}
1 & a & b \\
0 & 1 & c \\
0 & 0 & 1
\end{array}\right): a, b, c \in \mathbb{R}\right\}
$$

This is an exponential Lie group:

$$
\begin{array}{clc}
\exp : & \rightarrow & \\
& a p+c q+b z & \mapsto\left(\begin{array}{ccc}
1 & a & b \\
0 & 1 & c \\
0 & 0 & 1
\end{array}\right) .
\end{array}
$$

So we can apply Proposition 14 to construct the standard left-invariant notion of simplices in $H$, and Proposition 13 to integrate the Lie algebra 3 -cocycle $\gamma$ to a Lie group 3-cocycle $\int \gamma$. We therefore get a Lie 2-group, the Heisenberg Lie 2-group:

$$
\text { Heisenberg }=\text { String }_{\int \gamma}(H) .
$$

\section{$5 \quad$ Supergeometry and supergroups}

We would now like to generalize our work from Lie algebras and Lie groups to Lie superalgebras and supergroups. Of course, this means that we need a way to talk about Lie supergroups, their underlying supermanifolds, and the maps between supermanifolds. This task is made easier because we do not need the full machinery of supermanifold theory. Because our supergroups will be exponential, we only need to work with supermanifolds that are diffeomorphic to super vector spaces. Nonetheless, let us begin with a sketch of supermanifold theory from the perspective that suits us best, which could loosely be called the "functor of points" approach.

The rough geometric picture one should have of a supermanifold $M$ is that of an ordinary manifold with infinitesimal "superfuzz", or "superdirections", 
around each point. At the infinitesimal level, an ordinary manifold is merely a vector space - its tangent space at a point. In contrast, the tangent space to $M$ has a $\mathbb{Z}_{2}$-grading: tangent vectors which point along the underlying manifold of $M$ are taken to be even, while tangent vectors which point along the superdirections are taken to be odd.

At least infinitesimally, then, all supermanifolds look like super vector spaces,

$$
\mathbb{R}^{p \mid q}:=\mathbb{R}^{p} \oplus \mathbb{R}^{q},
$$

where $\mathbb{R}^{p}$ is even and $\mathbb{R}^{q}$ is odd. And indeed, just as ordinary manifolds are locally modeled on ordinary vector spaces, $\mathbb{R}^{n}$, supermanifolds are locally modeled on super vector spaces, $\mathbb{R}^{p \mid q}$. However, before we sketch how this works, let us introduce our main tool: the so-called "functor of points".

The basis for the functor of points is the Yoneda Lemma, a very general and fundamental fact from category theory:

Yoneda Lemma. Let $C$ be a category. The functor

$$
\begin{aligned}
& C \rightarrow \operatorname{Fun}\left(C^{\text {op }}, \text { Set }\right) \\
& x \mapsto \operatorname{Hom}(-, x)
\end{aligned}
$$

is a full and faithful embedding of $C$ into the category $\operatorname{Fun}\left(C^{\mathrm{op}}\right.$, Set $)$ of contravariant functors from $C$ to Set. This embedding is called the Yoneda embedding.

The upshot of this lemma is that, without losing any information, we can replace an object $x$ by a functor $\operatorname{Hom}(-, x)$, and a morphism $f: x \rightarrow y$ by a natural transformation

$$
\operatorname{Hom}(-, f): \operatorname{Hom}(-, x) \Rightarrow \operatorname{Hom}(-, y)
$$

of functors. Each component of this natural transformation is the "obvious" thing: for an object $z$, the function

$$
\operatorname{Hom}(z, f): \operatorname{Hom}(z, x) \rightarrow \operatorname{Hom}(z, y)
$$

just takes the morphism $g: z \rightarrow x$ to the morphism $f g: z \rightarrow y$.

On a more intuitive level, the functor of points tells us how to reconstruct a "space" $x \in C$ by probing it with every other space $z \in C$ - that is, by looking at all the ways in which $z$ maps into $x$, which forms the set $\operatorname{Hom}(z, x)$. The true power of the functor of points, however, arises when 
we can reconstruct $x$ without having to probe it will every $z$, but with $z$ from a manageable subcategory of $C$. And while it deviates slightly from the spirit of the Yoneda Lemma, we can shrink this subcategory still further if we allow $\operatorname{Hom}(z, x)$ to have more structure than that of a mere set. In fact, when $M$ is a supermanifold, we will consider probes $z$ for which $\operatorname{Hom}(z, M)$ is an ordinary manifold.

For what $z$ is $\operatorname{Hom}(z, M)$ a manifold? One clue is that when $M$ is an ordinary manifold, there is a manifold of ways to map a point into $M$ :

$$
M \cong \operatorname{Hom}\left(\mathbb{R}^{0}, M\right),
$$

but the space of maps from any higher-dimensional manifold to $M$ is generally not a finite-dimensional manifold in its own right. Similarly, when $M$ is a supermanifold, there is an ordinary manifold of ways to map a point into $M$ :

$$
M_{\mathbb{R}^{0 \mid 0}}=\operatorname{Hom}\left(\mathbb{R}^{0 \mid 0}, M\right) .
$$

One should think of this as the ordinary manifold one gets from $M$ by forgetting about the superdirections. But thanks to the superdirections, we now we have more ways of obtaining a manifold of maps to $M$ : there is an ordinary manifold of ways to map a point with $q$ superdirections into $M$ :

$$
M_{\mathbb{R}^{0 \mid q}}=\operatorname{Hom}\left(\mathbb{R}^{0 \mid q}, M\right) .
$$

So, for every supermanifold $M$, we get a functor:

$$
\begin{array}{ccc}
\operatorname{Hom}(-, M): \quad \text { SuperPoints }^{\text {op }} & \rightarrow & \operatorname{Man} \\
\mathbb{R}^{0 \mid q} & \mapsto \operatorname{Hom}\left(\mathbb{R}^{0 \mid q}, M\right)
\end{array}
$$

where SuperPoints is the category consisting of supermanifolds of the form $\mathbb{R}^{0 \mid q}$ and smooth maps between them. Of course, we have not yet said what this category is precisely, but one should think of $\mathbb{R}^{0 \mid q}$ as a supermanifold whose underlying manifold consists of one point, with $q$ infinitesimal superdirections - a "superpoint". Because this lets us probe the superdirections of $M$, this functor has enough information to completely reconstruct $M$. We will go further, however, and sketch how to define $M$ as a certain kind of functor from SuperPoints ${ }^{\text {op }}$ to Man.

This approach goes back to Schwarz [51] and Voronov [54], who used it to formalize the idea of "anticommuting coordinates" used in the physics literature. Since Schwarz, a number of other authors have developed the functor of points approach to supermanifolds, most recently Sachse [44] and 
Balduzzi et al. [11]. We will follow Sachse, who defines supermanifolds entirely in terms of their functors of points, rather than using sheaves.

\subsection{Supermanifolds}

\subsubsection{Super vector spaces as supermanifolds}

Let us now dive into supermathematics. Our main need is to define smooth maps between super vector spaces, but we will sketch the full definition of supermanifolds and the smooth maps between them. Just as an ordinary manifold is a space that is locally modeled on a vector space, a supermanifold is locally modeled on a super vector space. Since we will define a supermanifold $M$ as a functor

$$
M: \text { SuperPoints }^{\mathrm{op}} \rightarrow \text { Man, }
$$

we first need to say how to think of the simplest kind of supermanifold, a super vector space $V$, as such a functor:

$$
V: \text { SuperPoints }^{\text {op }} \rightarrow \text { Man. }
$$

But first we owe the reader a definition of the category of superpoints.

Recall from Section 3 that a super vector space is a $\mathbb{Z}_{2}$-graded vector space $V=V_{0} \oplus V_{1}$ where $V_{0}$ is called the even part, and $V_{1}$ is called the odd part. There is a symmetric monoidal category SuperVect which has:

- $\mathbb{Z}_{2}$-graded vector spaces as objects;

- Grade-preserving linear maps as morphisms;

- A tensor product $\otimes$ that has the following grading: if $V=V_{0} \oplus V_{1}$ and $W=W_{0} \oplus W_{1}$, then $(V \otimes W)_{0}=\left(V_{0} \otimes W_{0}\right) \oplus\left(V_{1} \otimes W_{1}\right)$ and $(V \otimes W)_{1}=\left(V_{0} \otimes W_{1}\right) \oplus\left(V_{1} \otimes W_{0}\right) ;$

- A braiding

$$
B_{V, W}: V \otimes W \rightarrow W \otimes V
$$

defined as follows: $v \in V$ and $w \in W$ are of grade $|v|$ and $|w|$, then

$$
B_{V, W}(v \otimes w)=(-1)^{|v||w|} w \otimes v .
$$

The braiding encodes the "the rule of signs": in any calculation, when two odd elements are interchanged, we introduce a minus sign. We write $\mathbb{R}^{p \mid q}$ for the super vector space with even part $\mathbb{R}^{p}$ and odd part $\mathbb{R}^{q}$. 
We define a supercommutative superalgebra to be a commutative algebra $A$ in the category SuperVect. More concretely, it is a real, associative algebra $A$ with unit which is $\mathbb{Z}_{2}$-graded:

$$
A=A_{0} \oplus A_{1},
$$

and is graded-commutative. That is:

$$
a b=(-1)^{|a||b|} b a,
$$

for all homogeneous elements $a, b \in A$, as required by the rule of signs. We define a homomorphism of superalgebras $f: A \rightarrow B$ to be an algebra homomorphism that respects the grading. So, there is a category SuperAlg with supercommutative superalgebras as objects, and homomorphisms of superalgebras as morphisms. Henceforth, we will assume all our superalgebras to be supercommutative unless otherwise indicated.

A particularly important example of a supercommutative superalgebra is a Grassmann algebra: a finite-dimensional exterior algebra

$$
A=\Lambda \mathbb{R}^{n},
$$

equipped with the grading:

$$
A_{0}=\Lambda^{0} \mathbb{R}^{n} \oplus \Lambda^{2} \mathbb{R}^{n} \oplus \cdots, \quad A_{1}=\Lambda^{1} \mathbb{R}^{n} \oplus \Lambda^{3} \mathbb{R}^{n} \oplus \cdots
$$

Let us write GrAlg for the category with Grassmann algebras as objects and homomorphisms of superalgebras as morphisms.

In fact, the Grassmann algebras are essential for our approach to supermanifold theory, because:

$$
\text { GrAlg }=\text { SuperPoints }{ }^{\text {op }}
$$

so rather than thinking of a supermanifold $M$ as a contravariant functor from SuperPoints to Man, we can view a supermanifold as a covariant functor:

$$
M: \text { GrAlg } \rightarrow \text { Man }
$$

To see why this is sensible, recall that a smooth map between ordinary manifolds

$$
\varphi: M \rightarrow N
$$


is the same as a homomorphism between their algebras of smooth functions which goes the other way:

$$
\varphi^{*}: C^{\infty}(N) \rightarrow C^{\infty}(M)
$$

By analogy, we expect something similar to hold for supermanifolds. In particular, a smooth map from a superpoint:

$$
\varphi: \mathbb{R}^{0 \mid q} \rightarrow M
$$

ought to be to the same as a homomorphism of their "superalgebras of smooth functions" which points the other way:

$$
\varphi^{*}: C^{\infty}(M) \rightarrow C^{\infty}\left(\mathbb{R}^{0 \mid q}\right) .
$$

But since $\mathbb{R}^{0 \mid q}$ is a purely odd super vector space, we define its algebra of smooth functions to be $\Lambda\left(\mathbb{R}^{q}\right)^{*}$. Intuitively, this is because $\mathbb{R}^{0 \mid q}$ is a supermanifold with $q$ "odd, anticommuting coordinates", given by the standard projections:

$$
\theta^{1}, \ldots, \theta^{q}: \mathbb{R}^{q} \rightarrow \mathbb{R}
$$

so a "smooth function" $f$ on $\mathbb{R}^{0 \mid q}$ should have a "power series expansion" that looks like:

$$
f=\sum_{i_{1}<i_{2}<\cdots<i_{k}} f_{i_{1} i_{2} \ldots i_{k}} \theta^{i_{1}} \wedge \theta^{i_{2}} \wedge \cdots \wedge \theta^{i_{k}} .
$$

where the coefficients $f_{i_{1} i_{2} \ldots i_{k}}$ are real. Such $f$ is precisely an element of $\Lambda\left(\mathbb{R}^{q}\right)^{*}$, so we define

$$
\operatorname{Hom}\left(\mathbb{R}^{0 \mid q}, M\right)=\operatorname{Hom}\left(C^{\infty}(M), \Lambda\left(\mathbb{R}^{q}\right)^{*}\right) .
$$

In this way, rather than thinking of $M$ as a functor:

$$
\begin{array}{ccc}
\operatorname{Hom}(-, M): \quad \text { SuperPoints }^{\text {op }} & \rightarrow & \operatorname{Man} \\
\mathbb{R}^{0 \mid q} & \mapsto \operatorname{Hom}\left(\mathbb{R}^{0 \mid q}, M\right)
\end{array}
$$

where Hom is in the category of supermanifolds (though we have not defined this), we think of $M$ as a functor:

$$
\begin{array}{rlr}
\operatorname{Hom}\left(C^{\infty}(M),-\right): \quad \operatorname{GrAlg} & \rightarrow & \operatorname{Man} \\
A & \mapsto \operatorname{Hom}\left(C^{\infty}(M), A\right)
\end{array}
$$

where Hom is in the category of superalgebras (which we have defined, though we have not defined $\left.C^{\infty}(M)\right)$. 
Since we have just given a slew of definitions, let us bring the discussion back down to earth with a concise summary:

- Every supermanifold is a functor:

$$
M: \operatorname{GrAlg} \rightarrow \text { Man, }
$$

though not every such functor is a supermanifold.

- Every smooth map of supermanifolds is a natural transformation:

$$
\varphi: M \rightarrow N
$$

though not every such natural transformation is a smooth map of supermanifolds.

Next, let us introduce some concise notation:

- Let us write $M_{A}$ for the value of $M$ on the Grassmann algebra $A$, and call this the $\boldsymbol{A}$-points of $M$.

- Let us write $M_{f}: M_{A} \rightarrow M_{B}$ for the smooth map induced by a homomorphism $f: A \rightarrow B$.

- Finally, we write $\varphi_{A}: M_{A} \rightarrow N_{A}$ for the smooth map which the natural transformation $\varphi$ gives between the $A$-points. We call $\varphi_{A}$ a component of the natural transformation $\varphi$.

With this background, we can now build up the theory of supermanifolds in perfect analogy to the theory of manifolds. First, we need to say how to think of our model spaces, the super vector spaces, as supermanifolds.

Indeed, given a finite-dimensional super vector space $V$, define the supermanifold associated to $\boldsymbol{V}$, or just the supermanifold $\boldsymbol{V}$ to be the functor:

$$
V: \operatorname{GrAlg} \rightarrow \text { Man }
$$

which takes:

- each Grassmann algebra $A$ to the vector space:

$$
V_{A}=(A \otimes V)_{0}=A_{0} \otimes V_{0} \oplus A_{1} \otimes V_{1}
$$

regarded as a manifold in the usual way; 
"ATMP-16-5-A4-HUE" — 2013/4/30 — 17:32 — page 1546 — \#62

- each homomorphism $f: A \rightarrow B$ of Grassmann algebras to the linear map $V_{f}: V_{A} \rightarrow V_{B}$ that is the identity on $V$ and $f$ on $A$ :

$$
V_{f}=(f \otimes 1)_{0}:(A \otimes V)_{0} \rightarrow(B \otimes V)_{0} .
$$

This map, being linear, is also smooth.

We take this definition because, roughly speaking, the set of $A$-points is the set of homomorphisms of superalgebras, $\operatorname{Hom}\left(C^{\infty}(V), A\right)$. By analogy with the ordinary manifold case, we expect that any such homomorphism is determined by its restriction to the "dense subalgebra" of polynomials:

$$
\operatorname{Hom}\left(C^{\infty}(V), A\right) \cong \operatorname{Hom}\left(\operatorname{Sym}\left(V^{*}\right), A\right),
$$

though here we are being very rough, because we have not assumed any topology on our superalgebras, so the term "dense subalgebra" is not meaningful. Since $\operatorname{Sym}\left(V^{*}\right)$ is the free supercommutative superalgebra on $V^{*}$, a homomorphism out of it is the same as a linear map of super vector spaces:

$$
\operatorname{Hom}\left(\operatorname{Sym}\left(V^{*}\right), A\right) \cong \operatorname{Hom}\left(V^{*}, A\right),
$$

where the first Hom is in SuperAlg and the second Hom is in SuperVect. Finally, because $V$ is finite-dimensional and linear maps of super vector spaces preserve grading, this last Hom is just:

$$
\operatorname{Hom}\left(V^{*}, A\right) \cong V_{0} \otimes A_{0} \oplus V_{1} \otimes A_{1} .
$$

which, up to a change of order in the factors, is how we defined $V_{A}$. This last set is a manifold in an obvious way: it is an ordinary, finite-dimensional, real vector space. In fact, it is just the even part of the super vector space $A \otimes V:$

$$
V_{A}=(A \otimes V)_{0},
$$

as we have noted in our definition.

Further, $V_{A}=A_{0} \otimes V_{0} \oplus A_{1} \otimes V_{1}$ is more than a mere vector space-it is an $A_{0}$-module. Moreover, given any linear map of super vector spaces:

$$
L: V \rightarrow W
$$

we get an $A_{0}$-module map between the $A$-points in a natural way:

$$
L_{A}=(1 \otimes L)_{0}:(A \otimes V)_{0} \rightarrow(A \otimes W)_{0} .
$$


"ATMP-16-5-A4-HUE" — 2013/4/30 — 17:32 — page 1547 — \#63

Indeed, $L$ induces a natural transformation between the supermanifold $V$ and the supermanifold $W$. That is, given any homomorphism $f: A \rightarrow B$ of Grassmann algebras, the following square commutes:

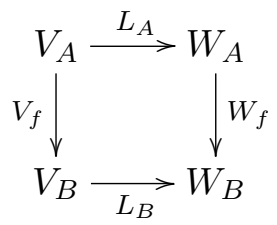

We therefore have a functor

$$
\text { SuperVect } \rightarrow \text { Fun(GrAlg, Man) }
$$

which takes super vector spaces to their associated supermanifolds, and linear transformations to natural transformations between supermanifolds. For future reference, we note this fact in a proposition:

Proposition 19. There is a faithful functor:

$$
\text { SuperVect } \rightarrow \text { Fun(GrAlg, Man) }
$$

that takes a super vector space $V$ to the supermanifold $V$ whose A-points are:

$$
V_{A}=(A \otimes V)_{0},
$$

and takes a linear map of super vector spaces:

$$
L: V \rightarrow W
$$

to the natural transformation whose components are:

$$
L_{A}=(1 \otimes L)_{0}:(A \otimes V)_{0} \rightarrow(A \otimes W)_{0} .
$$

In the above, $A$ is a Grassmann algebra and the tensor product takes place in SuperVect.

Proof. It is easy to check that this defines a functor. Faithfulness follows from a more general result in Sachse [44]; cf. Proposition 3.1.

While this functor is faithful, it is far from full; in particular, it misses all of the "smooth maps" between super vector spaces which do not come from a linear map. We define these additional maps now. 
Infinitesimally, all smooth maps should be like a linear map $L: V \rightarrow W$, so given two finite-dimensional super vector spaces $V$ and $W$, we define a smooth map between super vector spaces:

$$
\varphi: V \rightarrow W
$$

to be a natural transformation between the supermanifolds $V$ and $W$ such that the derivative

$$
\left(\varphi_{A}\right)_{*}: T_{x} V_{A} \rightarrow T_{\varphi_{A}(x)} W_{A}
$$

is $A_{0}$-linear at each $A$-point $x \in V_{A}$, where the $A_{0}$-module structure on each tangent space comes from the canonical identification of a vector space with its tangent space:

$$
T_{x} V_{A} \cong V_{A}, \quad T_{\varphi(x)} W_{A} \cong W_{A}
$$

Note that each component $\varphi_{A}: V_{A} \rightarrow W_{A}$ is smooth in the ordinary sense, by virtue of living in the category of smooth manifolds. We say that a smooth map $\varphi_{A}: V_{A} \rightarrow W_{A}$ whose derivative is $A_{0}$-linear at each point is $A_{0}$-smooth for short.

Finally, note that there is a supermanifold:

$$
\text { 1: GrAlg } \rightarrow \text { Man, }
$$

which takes each Grassmann algebra to the one-point manifold. We call this the one-point supermanifold, and note that it is the supermanifold associated to the super vector space $\mathbb{R}^{0 \mid 0}$. The one-point supermanifold is the terminal object in the category of supermanifolds, whose definition we now describe.

\subsubsection{Supermanifolds in general}

The last section treated the special kind of a supermanifold of greatest interest to us: the supermanifold associated to a super vector space.

Nonetheless, we now sketch how to define a general supermanifold, $M$. Since $M$ will be locally isomorphic to a super vector space $V$, it helps to have local pieces of $V$ to play the same role that open subsets of $\mathbb{R}^{n}$ play for ordinary manifolds. So, fix a super vector space $V$, and let $U \subseteq V_{0}$ be 
"ATMP-16-5-A4-HUE" — 2013/4/30 — 17:32 — page 1549 — \#65

open. The superdomain over $U$ is the functor:

$$
\mathcal{U}: \text { GrAlg } \rightarrow \text { Man }
$$

that takes each Grassmann algebra $A$ to the following open subset of $V_{A}$ :

$$
\mathcal{U}_{A}=V_{\epsilon_{A}}^{-1}(U)
$$

where $\epsilon_{A}: A \rightarrow \mathbb{R}$ is the projection of the Grassmann algebra $A$ that kills all nilpotent elements. We say that $\mathcal{U}$ is a superdomain in $\boldsymbol{V}$, and write $\mathcal{U} \subseteq V$

If $\mathcal{U} \subseteq V$ and $\mathcal{U}^{\prime} \subseteq W$ are two superdomains in super vector spaces $V$ and $W$, a smooth map of superdomains is a natural transformation:

$$
\varphi: \mathcal{U} \rightarrow \mathcal{U}^{\prime}
$$

such that for each Grassmann algebra $A$, the component on $A$-points is smooth:

$$
\varphi_{A}: \mathcal{U}_{A} \rightarrow \mathcal{U}^{\prime}{ }_{A}
$$

and the derivative:

$$
\left(\varphi_{A}\right)_{*}: T_{x} \mathcal{U}_{A} \rightarrow T_{\varphi_{A}(x)} \mathcal{U}^{\prime}{ }_{A}
$$

is $A_{0}$-linear at each $A$-point $x \in \mathcal{U}_{A}$, where the $A_{0}$-module structure on each tangent space comes from the canonical identification with the ambient vector spaces:

$$
T_{x} \mathcal{U}_{A} \cong V_{A}, \quad T_{\varphi(x)} \mathcal{U}_{A}^{\prime} \cong W_{A}
$$

Again, we say that a smooth map $\varphi_{A}: \mathcal{U}_{A} \rightarrow \mathcal{U}^{\prime}{ }_{A}$ whose derivative is $A_{0^{-}}$ linear at each point is $\boldsymbol{A}_{\mathbf{0}}$-smooth for short.

At long last, a supermanifold is a functor

$$
M: \operatorname{GrAlg} \rightarrow \text { Man }
$$

equipped with an atlas

$$
\left(\mathcal{U}_{\alpha}, \varphi_{\alpha}: \mathcal{U} \rightarrow M\right)
$$

where each $\mathcal{U}_{\alpha}$ is a superdomain, each $\varphi_{\alpha}$ is a natural transformation, and one can define transition functions that are smooth maps of superdomains. 
"ATMP-16-5-A4-HUE" — 2013/4/30 — 17:32 — page 1550 — \#66

Finally, a smooth map of supermanifolds is a natural transformation:

$$
\psi: M \rightarrow N
$$

which induces smooth maps between the superdomains in the atlases. Equivalently, each component

$$
\varphi_{A}: M_{A} \rightarrow N_{A}
$$

is $\boldsymbol{A}_{\mathbf{0}}$-smooth: it is smooth and its derivative

$$
\left(\varphi_{A}\right)_{*}: T_{x} M_{A} \rightarrow T_{\varphi_{A}(x)} N_{A}
$$

is $A_{0}$-linear at each $A$-point $x \in M_{A}$, where the $A_{0}$-module structure on each tangent space comes from the superdomains in the atlases. Thus, there is a category SuperMan of supermanifolds. See Sachse [44] for more details.

\subsection{Supergroups from nilpotent Lie superalgebras}

We now describe a procedure to integrate a nilpotent Lie superalgebra to a Lie supergroup. This is a partial generalization of Lie's Third Theorem, which describes how any Lie algebra can be integrated to a Lie group. In fact, the full theorem generalizes to Lie supergroups [53], but we do not need it here.

Recall from Section 3 that a Lie superalgebra $\mathfrak{g}$ is a Lie algebra in the category of super vector spaces. More concretely, it is a super vector space $\mathfrak{g}=\mathfrak{g}_{0} \oplus \mathfrak{g}_{1}$, equipped with a graded-antisymmetric bracket:

$$
[-,-]: \Lambda^{2} \mathfrak{g} \rightarrow \mathfrak{g},
$$

which satisfies the Jacobi identity up to signs:

$$
[X,[Y, Z]]=[[X, Y], Z]+(-1)^{|X||Y|}[Y,[X, Z]] .
$$

for all homogeneous $X, Y, Z \in \mathfrak{g}$. A Lie superalgebra $\mathfrak{n}$ is called $\mathbf{k}$-step nilpotent if any $k$ nested brackets vanish, and it is called nilpotent if it is $k$-step nilpotent for some $k$. Nilpotent Lie superalgebras can be integrated to a unique supergroup $N$ defined on the same underlying super vector space $\mathfrak{n}$.

A Lie supergroup, or supergroup, is a group object in the category of supermanifolds. That is, it is a supermanifold $G$ equipped with the following maps of supermanifolds: 
- multiplication, $m: G \times G \rightarrow G$;

- inverse, inv: $G \rightarrow G$;

- identity, id: $1 \rightarrow G$, where 1 is the one-point supermanifold;

such that the following diagrams commute, encoding the usual group axioms:

- the associative law:

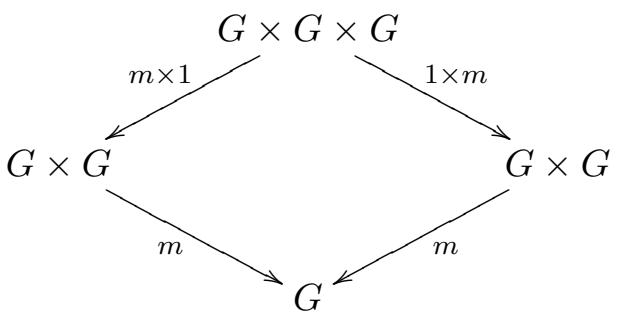

- the right and left unit laws:

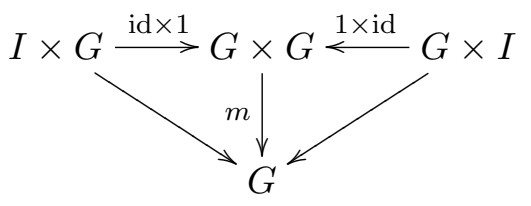

- the right and left inverse laws:
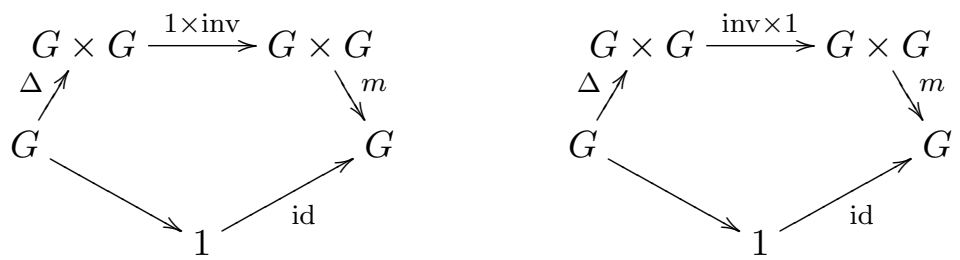

where $\Delta: G \rightarrow G \times G$ is the diagonal map. In addition, a supergroup is abelian if the following diagram commutes:

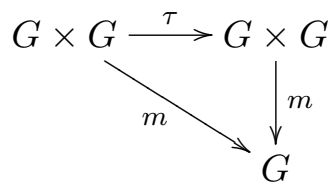

where $\tau: G \times G \rightarrow G \times G$ is the twist map. Using $A$-points, it is defined to be:

$$
\tau_{A}(x, y)=(y, x),
$$

for $(x, y) \in G_{A} \times G_{A}$. 
Examples of supergroups arise easily from Lie groups. We can regard any ordinary manifold as a supermanifold, and so any Lie group $G$ is also a supergroup. In this way, any classical Lie group, such as $\mathrm{SO}(n), \mathrm{SU}(n)$ and $\operatorname{Sp}(n)$, becomes a supergroup.

To obtain more interesting examples, we will integrate a nilpotent Lie superalgebra, $\mathfrak{n}$ to a supergroup $N$. For any Grassmann algebra $A$, the bracket

$$
[-,-]: \Lambda^{2} \mathfrak{n} \rightarrow \mathfrak{n}
$$

induces an $A_{0}$-linear map between the $A$-points:

$$
[-,-]_{A}: \Lambda^{2} \mathfrak{n}_{A} \rightarrow \mathfrak{n}_{A},
$$

where $\Lambda^{2} \mathfrak{n}_{A}$ denotes the exterior square of the $A_{0}$-module $\mathfrak{n}_{A}$. Thus $[-,-]_{A}$ is antisymmetric, and it easy to check that it makes $\mathfrak{n}_{A}$ into a Lie algebra which is also nilpotent.

On each such $A_{0}$-module $\mathfrak{n}_{A}$, we can thus define a Lie group $N_{A}$ where the multplication is given by the Baker-Campbell-Hausdorff formula, inversion by negation, and the identity is 0 . Because we want to write the group $N_{A}$ multiplicatively, we write $\exp _{A}: n_{A} \rightarrow N_{A}$ for the identity map, and then define the multiplication, inverse and identity:

$$
m_{A}: N_{A} \times N_{A} \rightarrow N_{A}, \quad \operatorname{inv}_{A}: N_{A} \rightarrow N_{A}, \quad \operatorname{id}_{A}: 1_{A} \rightarrow N_{A},
$$

as follows:

$$
\begin{aligned}
m_{A}\left(\exp _{A}(X), \exp _{A}(Y)\right) & =\exp _{A}(X) \exp _{A}(Y) \\
& =\exp _{A}\left(X+Y+\frac{1}{2}[X, Y]_{A}+\cdots\right) \\
\operatorname{inv}_{A}\left(\exp _{A}(X)\right) & =\exp _{A}(X)^{-1}=\exp _{A}(-X), \\
\operatorname{id}_{A}(1) & =1=\exp _{A}(0),
\end{aligned}
$$

for any $A$-points $X, Y \in \mathfrak{n}_{A}$, where the first 1 in the last equation refers to the single element of $1_{A}$. But it is clear that all of these maps are natural in $A$. Furthermore, they are all $A_{0}$-smooth, because as polynomials with coefficients in $A_{0}$, they are smooth with derivatives that are $A_{0}$-linear. They thus define smooth maps of supermanifolds:

$$
m: N \times N \rightarrow N, \quad \text { inv }: N \rightarrow N, \quad \text { id }: 1 \rightarrow N,
$$

where $N$ is the supermanifold $\mathfrak{n}$. And because each of the $N_{A}$ is a group, $N$ is a supergroup. We have thus proved: 
Proposition 20. Let $\mathfrak{n}$ be a nilpotent Lie superalgebra. Then there is a supergroup $N$ defined on the supermanifold $\mathfrak{n}$, obtained by integrating the nilpotent Lie algebra $\mathfrak{n}_{A}$ with the Baker-Campbell-Hausdorff formula for all Grassmann algebras A. More precisely, we define the maps:

$$
m: N \times N \rightarrow N, \quad \text { inv }: N \rightarrow N, \quad \text { id }: 1 \rightarrow N
$$

by defining them on A-points as follows:

$$
\begin{gathered}
m_{A}\left(\exp _{A}(X), \exp _{A}(Y)\right)=\exp _{A}(Z(X, Y)) \\
\operatorname{inv}_{A}\left(\exp _{A}(X)\right)=\exp _{A}(-X) \\
\operatorname{id}_{A}(1)=\exp _{A}(0)
\end{gathered}
$$

where

$$
\exp : \mathfrak{n} \rightarrow N
$$

is the identity map of supermanifolds, and:

$$
Z(X, Y)=X+Y+\frac{1}{2}[X, Y]_{A}+\cdots
$$

denotes the Baker-Campbell-Hausdorff series on $\mathfrak{n}_{A}$, which terminates because $\mathfrak{n}_{A}$ is nilpotent.

Experience with ordinary Lie theory suggests that, in general, there will be more than one supergroup which has Lie superalgebra $\mathfrak{n}$. To distinguish the one above, we call $N$ the exponential supergroup of $\mathfrak{n}$.

\subsection{The Poincaré supergroup}

We can use the result of the last section to construct our favorite supergroup: the Poincaré supergroup, $\operatorname{SISO}(n+1,1)$, the supergroup of symmetries of "Minkowski superspacetime". First, we construct superspacetime, using a familiar trick: just as we can identify the group of spacetime translations with spacetime itself, we can identify the supergroup of supertranslations, $T$, with superspacetime itself. So let us build $T$, and define superspacetime to be $T$. 
We have already met the Lie superalgebra of $T$, at least for the special dimensions where superstring theory makes sense. In Section 3.1.3, we constructed the supertranslation algebra $\mathcal{T}$ for spacetimes of dimension $n+2=3,4,6$ and 10. This is the Lie superalgebra whose even part consists of vectors, $\mathcal{T}_{0}=V$, whose odd part consists of spinors, $\mathcal{T}_{1}=S_{+}$, and whose Lie bracket vanishes except for the $\operatorname{Spin}(n+1,1)$-equivariant map:

$$
\cdot: \mathrm{Sym}^{2} S_{+} \rightarrow V \text {. }
$$

Thanks to the near triviality of the bracket, $\mathcal{T}$ is nilpotent. Thus we can use Proposition 20 to construct the exponential supergroup $T$ of $\mathcal{T}$. We think of this as the supergroup of translations on "superspacetime". In fact, we define Minkowski superspacetime to be the supergroup $T$.

As we noted in the last section, we can think of the Lorentz group $\operatorname{Spin}(n+1,1)$ as a supergroup in a trivial way. Note that $\operatorname{Spin}(n+1,1)$ acts on $\mathcal{T}$ and hence $T$ by automorphism, so we can define the Poincaré supergroup, $\operatorname{SISO}(n+1,1)$, to be the semidirect product:

$$
\operatorname{SISO}(n+1,1)=\operatorname{Spin}(n+1,1) \ltimes T .
$$

\section{Lie 2-supergroups from supergroup cohomology}

We saw in Section 2 that 3-cocycles in Lie group cohomology allow us to construct Lie 2-groups. We now generalize this to supergroups. The most significant barrier is that we now work internally to the category of supermanifolds instead of the much more familiar category of smooth manifolds. Our task is to show that this change of categories does not present a problem. The main obstacle is that the category of supermanifolds is not a concrete category: morphisms are determined not by their value on the underlying set of a supermanifold, but by their value on $A$-points for all Grassmann algebras $A$.

The most common approach is to define morphisms without reference to elements, and to define equations between morphisms using commutative diagrams. This is how we gave the definition of smooth bicategory, except that we found it convenient to state the pentagon and triangle identities using elements. As an alternative to commutative diagrams, for supermanifolds, one can use $A$-points to define morphisms and specify equations between them. This tends to make equations look friendlier, because they look like equations between functions. We shall use this approach. 
First, let us define the cohomology of a supergroup $G$ with coefficients in an abelian supergroup $H$, on which $G$ acts by automorphism. This means that we have a morphism of supermanifolds:

$$
\alpha: G \times H \rightarrow H,
$$

which, for any Grassmann algebra $A$, induces an action of the group $G_{A}$ on the abelian group $H_{A}$ :

$$
\alpha_{A}: G_{A} \times H_{A} \rightarrow H_{A} .
$$

For this action to be by automorphism, we require:

$$
\alpha_{A}(g)\left(h+h^{\prime}\right)=\alpha_{A}(g)(h)+\alpha_{A}(g)\left(h^{\prime}\right),
$$

for all $A$-points $g \in G_{A}$ and $h, h^{\prime} \in H_{A}$.

We define supergroup cohomology using the supergroup cochain complex, $C^{\bullet}(G, H)$, which at level $p$ just consists of the set of maps from $G^{p}$ to $H$ as supermanifolds:

$$
C^{p}(G, H)=\left\{f: G^{p} \rightarrow H\right\} .
$$

Addition on $H$ makes $C^{p}(G, H)$ into an abelian group for all $p$. The differential is given by the usual formula, but using $A$-points:

$$
\begin{aligned}
d f_{A}\left(g_{1}, \ldots, g_{p+1}\right)= & g_{1} f_{A}\left(g_{2}, \ldots, g_{p+1}\right) \\
& +\sum_{i=1}^{p}(-1)^{i} f_{A}\left(g_{1}, \ldots, g_{i} g_{i+1}, \ldots, g_{p+1}\right) \\
& +(-1)^{p+1} f_{A}\left(g_{1}, \ldots, g_{p}\right)
\end{aligned}
$$

where $g_{1}, \ldots, g_{p+1} \in G_{A}$ and the action of $g_{1}$ is given by $\alpha_{A}$. Noting that $f_{A}, \alpha_{A}$, multiplication and + are all:

- natural in $A$;

- $A_{0}$-smooth: smooth with derivatives that are $A_{0}$-linear;

we see that $d f_{A}$ is:

- natural in $A$;

- $A_{0}$-smooth: smooth with a derivative which is $A_{0}$-linear; 
so it indeed defines a map of supermanifolds:

$$
d f: G^{p+1} \rightarrow H .
$$

Furthermore, it is immediate that:

$$
d^{2} f_{A}=0
$$

for all $A$, and thus

$$
d^{2} f=0
$$

So $C^{\bullet}(G, H)$ is truly a cochain complex. Its cohomology $H^{\bullet}(G, H)$ is the supergroup cohomology of $\boldsymbol{G}$ with coefficients in $\boldsymbol{H}$. Of course, if $d f=0, f$ is called a cocycle, and $f$ is normalized if

$$
f_{A}\left(g_{1}, \ldots, g_{p}\right)=0
$$

for any Grassmann algebra $A$, whenever one of the $A$-points $g_{1}, \ldots, g_{p}$ is 1 . When $H=\mathbb{R}$, we omit reference to it, and write $C^{\bullet}(G, \mathbb{R})$ as $C^{\bullet}(G)$.

A super bicategory $B$ has

- a supermanifold of objects $B_{0}$;

- a supermanifold of morphisms $B_{1}$;

- a supermanifold of 2-morphisms $B_{2}$;

equipped with maps of supermanifolds as described in Definition 4: source, target, identity-assigning, horizontal composition, vertical composition, associator and left and right unitors all maps of supermanifolds, and satisfying the same axioms as a smooth bicategory. The associator satisfies the pentagon identity, which we state in terms of $A$-points: the following pentagon commutes:

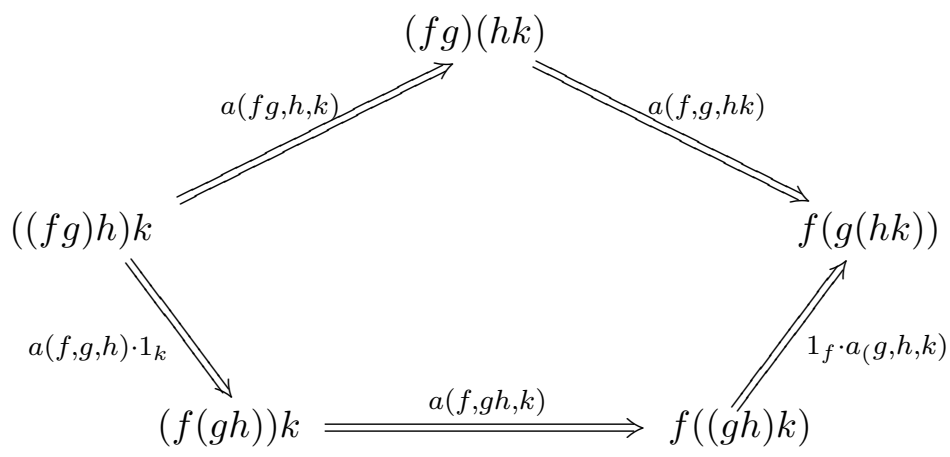


for any "composable quadruple of morphisms":

$$
(f, g, h, k) \in\left(B_{1} \times{ }_{B_{0}} B_{1} \times_{B_{0}} B_{1} \times_{B_{0}} B_{1}\right)_{A} .
$$

Similarly, the associator and left and right unitors satisfy the triangle identity, which we state in terms of $A$-points: the following triangle commutes:

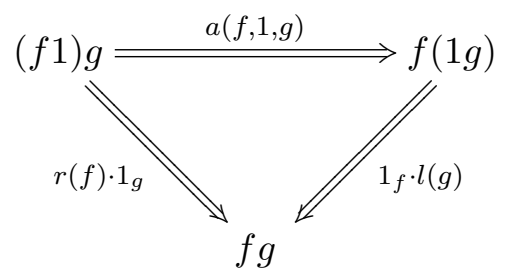

for any "composable pair of morphisms":

$$
(f, g) \in\left(B_{1} \times_{B_{0}} B_{1}\right)_{A} .
$$

We can similarly speak of super categories, smooth functors between super categories, and smooth natural isomorphisms between smooth functors, simply by taking the appropriate definition from Section 2 and replacing every manifold in sight with a supermanifold.

Finally, to talk about 2-supergroups, we will need to talk about inverses. Again, we can do this in exactly the same way as in the smooth case, but replacing points with $A$-points. We say that the 2 -morphisms in a super bicategory $B$ have smooth strict inverses if there exists a smooth map from 2-morphisms to 2-morphisms:

$$
\operatorname{inv}_{2}: B_{2} \rightarrow B_{2}
$$

such that inv $_{2}$ assigns each 2-morphism to its strict inverse. Stated in terms of $A$-points, this means:

$$
\alpha^{-1} \circ \alpha=1, \quad \alpha \circ \alpha^{-1}=1 .
$$

for all $\alpha \in\left(B_{2}\right)_{A}$. Likewise, we say that the morphisms in $B$ have smooth weak inverses if there exist smooth maps:

$$
\operatorname{inv}_{1}: B_{1} \rightarrow B_{1}, \quad e: B_{1} \rightarrow B_{2}, \quad u: B_{1} \rightarrow B_{2}
$$

such that inv 1 provides a smooth choice of weak inverse and $u$ and $e$ provide smooth choices of 2-isomorphisms that "weaken" the left and right inverse 
laws. In terms of $A$-points:

$$
e(f): f^{-1} \cdot f \Rightarrow 1, \quad u(f): f \cdot f^{-1} \Rightarrow 1 .
$$

for each $f \in\left(B_{1}\right)_{A}$.

A 2-supergroup is a super bicategory with one object (more precisely, the one-point supermanifold), whose morphisms have smooth weak inverses and whose 2-morphisms have smooth strict inverses. As we did with Lie 2-groups, we can construct examples of 2-supergroups from cocycles: given a normalized $H$-valued 3-cocycle $a$ on $G$, we can construct a 2 -supergroup $\operatorname{String}_{a}(G, H)$ in the same way we constructed the Lie 2 -group $\operatorname{String}_{a}(G, H)$ when $G$ and $H$ were Lie groups, by just deleting every reference to elements of $G$ or $H$ :

- The supermanifold of objects is the one-point supermanifold, 1.

- The supermanifold of morphisms is the supergroup $G$, with composition given by the multiplication:

$$
\cdot G \times G \rightarrow G .
$$

The source and target maps are the unique maps to the one-point supermanifold. The identity-assigning map is the identity-assigning map for $G$ :

$$
\text { id }: 1 \rightarrow G \text {. }
$$

- The supermanifold of 2-morphisms is $G \times H$. The source and target maps are both the projection map to $G$. The identity assigning map comes from the identity-assigning map for $H$ :

$$
1 \times \text { id }: G \times 1 \rightarrow G \times H .
$$

- Vertical composition of 2-morphisms is given by addition in $H$ :

$$
1 \times+: G \times H \times H \rightarrow G \times H,
$$

where we have used the fact that the pullback of 2-morphisms over morphisms is trivially:

$$
(G \times H) \times{ }_{1}(G \times H) \cong G \times H \times H .
$$

Horizontal composition, $\cdot$, given by the multiplication on the semidirect product:

$$
\cdot:(G \ltimes H) \times(G \ltimes H) \rightarrow G \ltimes H .
$$


- The left and right unitors are trivial.

- The associator is given by the 3-cocycle $a: G^{3} \rightarrow H$, where the source (and target) is understood to come from multiplication on $G$.

A slim 2-supergroup is one of this form. It remains to check that it is, indeed, a 2-supergroup.

Proposition 21. String $(G, H)$ is a 2-supergroup: a super bicategory with one object where all morphisms have smooth weak inverses and all 2-morphisms have smooth strict inverses.

Proof. This proof is a duplicate of the proof of Proposition 5, but with $A$-points instead of elements.

\section{$7 \quad$ Integrating nilpotent Lie $n$-superalgebras}

We now generalize our technique for integrating cocycles from nilpotent Lie algebras to nilpotent Lie superalgebras. Those familiar with supermanifold theory may find it surprising that this is possible - the theory of differential forms is very different for supermanifolds than for manifolds, and integrating differential forms on a manifold was crucial to our method in Section 4.1. But we can sidestep this issue on a supergroup $N$ by considering $A$-points for any Grassmann algebra $A$. Then $N_{A}$ is a manifold, so the usual theory of differential forms applies.

Here is how we will proceed. Fixing a nilpotent Lie superalgebra $\mathfrak{n}$ with exponential supergroup $N$, we can use Proposition 19 turn any even Lie superalgebra cochain $\omega$ on $\mathfrak{n}$ into a Lie algebra cochain $\omega_{A}$ on $\mathfrak{n}_{A}$. We then use the techniques in Section 4.1 to turn $\omega_{A}$ into a Lie group cochain $\int \omega_{A}$ on $N_{A}$. Checking that $\int \omega_{A}$ is natural in $A$ and $A_{0}$-smooth, this defines a supergroup cochain $\int \omega$ on $N$.

As we saw in Proposition 19, any map of super vector spaces becomes an $A_{0}$-linear map on $A$-points. We have already touched on the way this interacts with symmetry: for a Lie superalgebra $\mathfrak{g}$, the graded-antisymmetric bracket

$$
[-,-]: \Lambda^{2} \mathfrak{g} \rightarrow \mathfrak{g}
$$

becomes an honest antisymmetric bracket on $A$-points:

$$
[-,-]_{A}: \Lambda^{2} \mathfrak{g}_{A} \rightarrow \mathfrak{g}_{A} .
$$

More generally, we have: 
Lemma 22. Graded-symmetric maps of super vector spaces:

$$
f: \operatorname{Sym}^{p} V \rightarrow W
$$

induce symmetric maps on A-points:

$$
f_{A}: \operatorname{Sym}^{p} V_{A} \rightarrow W_{A},
$$

defined by:

$$
f_{A}\left(a_{1} v_{1}, \ldots, a_{p} v_{p}\right)=a_{p} \cdots a_{1} f\left(v_{1}, \ldots, v_{p}\right),
$$

where $\mathrm{Sym}^{p} V_{A}$ is the symmetric power of $V_{A}$ as an $A_{0}$-module and $a_{i} \in A$, $v_{i} \in V$ are of matching parity. Similarly, graded-antisymmetric maps of super vector spaces:

$$
f: \Lambda^{p} V \rightarrow W
$$

induce antisymmetric maps on A-points:

$$
f_{A}: \Lambda^{p} V_{A} \rightarrow W_{A},
$$

defined by:

$$
f_{A}\left(a_{1} v_{1}, \ldots, a_{p} v_{p}\right)=a_{p} \cdots a_{1} f\left(v_{1}, \ldots, v_{p}\right),
$$

where $\Lambda^{p} V_{A}$ is the exterior power $V_{A}$ as an $A_{0}$-module and $a_{i} \in A, v_{i} \in V$ are of matching parity.

Proof. This is straightforward and we leave it to the reader.

Recall from Section 3 that the cohomology of our nilpotent Lie superalgebra $\mathfrak{n}$ is computed from the complex of linear maps:

$$
C^{p}(\mathfrak{n})=\left\{\omega: \Lambda^{p} \mathfrak{n} \rightarrow \mathbb{R}, \text { linear }\right\} .
$$

Each level of this complex is a super vector space, where the parity preserving maps are even and the parity reversing maps are odd. Only the even cochains, however, are honest morphisms of super vector spaces to which we can apply the above proposition. For this reason, we will now restrict our attention to the subcomplex of even cochains:

$$
C_{0}^{p}(\mathfrak{n})=\left\{\omega: \Lambda^{p} \mathfrak{n} \rightarrow \mathbb{R}, \text { linear, parity-preserving }\right\} .
$$

Now we need to show that even Lie superalgebra cochains $\omega$ on $\mathfrak{n}$ give rise to Lie algebra cochains $\omega_{A}$ on the $A$-points $\mathfrak{n}_{A}$. In fact, this works for 
any Lie superalgebra, but there is one twist: because $\mathfrak{n}_{A}$ is an $A_{0}$-module, $\omega: \Lambda^{p} \mathfrak{n} \rightarrow \mathbb{R}$ gives rise to an $A_{0}$-linear map:

$$
\omega_{A}: \Lambda^{p} \mathfrak{n}_{A} \rightarrow A_{0},
$$

using the fact that $\mathbb{R}_{A}=A_{0}$. So, we need to say how to do Lie algebra cohomology with coefficients in $A_{0}$. It is just a straightforward generalization of cohomology with coefficients in $\mathbb{R}$.

Indeed, any Lie superalgebra $\mathfrak{g}$ induces a Lie algebra structure on $\mathfrak{g}_{A}$ where the bracket is $A_{0}$-bilinear. We say that $\mathfrak{g}_{A}$ is an $\boldsymbol{A}_{\mathbf{0}}$-Lie algebra. Given any $A_{0}$-Lie algebra $\mathfrak{g}_{A}$, we define its cohomology with the $\boldsymbol{A}_{\mathbf{0}}$-Lie algebra cochain complex, which at level $p$ consists of antisymmetric $A_{0^{-}}$ multilinear maps:

$$
C^{p}\left(\mathfrak{g}_{A}\right)=\left\{\omega: \Lambda^{p} \mathfrak{g}_{A} \rightarrow A_{0}\right\} .
$$

We define $d$ on this complex in exactly the same way we define $d$ for $\mathbb{R}$ valued Lie algebra cochains. This makes $C^{\bullet}\left(\mathfrak{g}_{A}\right)$ into a cochain complex, and the cohomology of an $\boldsymbol{A}_{\mathbf{0}}$-Lie algebra with coefficients in $\boldsymbol{A}_{0}$ is the cohomology of this complex.

Proposition 23. Let $\mathfrak{g}$ be a Lie superalgebra, and let $\mathfrak{g}_{A}$ be the $A_{0}$-Lie algebra of its A-points. Then there is a cochain map:

$$
C_{0}^{\bullet}(\mathfrak{g}) \rightarrow C^{\bullet}\left(\mathfrak{g}_{A}\right)
$$

given by taking the even p-cochain $\omega$

$$
\omega: \Lambda^{p} \mathfrak{g} \rightarrow \mathbb{R}
$$

to the induced $A_{0}$-linear map $\omega_{A}$ :

$$
\omega_{A}: \Lambda^{p} \mathfrak{g}_{A} \rightarrow A_{0},
$$

where $\Lambda^{p} \mathfrak{g}_{A}$ denotes the pth exterior power of $\mathfrak{g}_{A}$ as an $A_{0}$-module.

Proof. We need to show:

$$
d\left(\omega_{A}\right)=(d \omega)_{A} .
$$

Since these are both linear maps on $\Lambda^{p+1}\left(\mathfrak{g}_{A}\right)$, it suffices to check that they agree on generators, which are of the form:

$$
a_{1} X_{1} \wedge a_{2} X_{2} \wedge \cdots \wedge a_{p+1} X_{p+1}
$$


for $a_{i} \in A$ and $X_{i} \in \mathfrak{g}$ of matching parity. By definition:

$$
\begin{aligned}
& (d \omega)_{A}\left(a_{1} X_{1} \wedge a_{2} X_{2} \wedge \cdots \wedge a_{p+1} X_{p+1}\right) \\
& =a_{p+1} a_{p} \cdots a_{1} d \omega\left(X_{1} \wedge X_{2} \wedge \cdots \wedge X_{p+1}\right) .
\end{aligned}
$$

On the other hand, to compute $d\left(\omega_{A}\right)$, we need to apply the formula for $d$ to obtain the intimidating expression:

$$
\begin{aligned}
d\left(\omega_{A}\right)\left(a_{1} X_{1}, \ldots, a_{p+1} X_{p+1}\right)= & \sum_{i<j}(-1)^{i+j} \omega_{A}\left(\left[a_{i} X_{i}, a_{j} X_{j}\right]_{A}, a_{1} X_{1}, \ldots,\right. \\
& \left.\widehat{a_{i} X_{i}}, \ldots, \widehat{a_{j} X_{j}}, \ldots, a_{p+1} X_{p+1}\right) \\
= & \sum_{i<j}(-1)^{i+j} a_{p+1} \cdots \hat{a}_{j} \cdots \hat{a}_{i} \cdots a_{1} a_{j} a_{i} \omega\left(\left[X_{i}, X_{j}\right],\right. \\
& \left.X_{1}, \ldots, \hat{X}_{i}, \ldots, \hat{X}_{j}, \ldots, X_{p+1}\right) .
\end{aligned}
$$

If we reorder the each of the coefficients $a_{p+1} \cdots \hat{a}_{j} \cdots \hat{a}_{i} \cdots a_{1} a_{j} a_{i}$ to $a_{p+1} \cdots$ $a_{2} a_{1}$ at the cost of introducing still more signs, we can factor all of the $a_{i} \mathrm{~s}$ out of the summation to obtain:

$$
\begin{gathered}
a_{p+1} \cdots a_{2} a_{1} \sum_{i<j}(-1)^{i+j}(-1)^{\left|X_{i}\right|\left|X_{j}\right|} \epsilon_{1}^{i-1}(i) \epsilon_{1}^{j-1}(j) \\
\times \omega\left(\left[X_{i}, X_{j}\right], X_{1}, \ldots, \hat{X}_{i}, \ldots, \hat{X}_{j}, \ldots, X_{p+1}\right) \\
=a_{p+1} \cdots a_{2} a_{1} d \omega\left(X_{1} \wedge X_{2} \wedge \cdots \wedge X_{p+1}\right) .
\end{gathered}
$$

Note that the first two lines are a single quantity, the product of $a_{p+1} \cdots a_{1}$ and a large summation. The last line is $(d \omega)_{A}\left(a_{1} X_{1} \wedge \cdots \wedge a_{p+1} X_{p+1}\right)$, as desired.

This proposition says that from any even Lie superalgebra cocycle on $\mathfrak{n}$ we obtain a Lie algebra cocycle on $\mathfrak{n}_{A}$, albeit now valued in $A_{0}$. Since $N_{A}$ is an exponential Lie group with Lie algebra $\mathfrak{n}_{A}$, we can apply the techniques we developed in Section 4.1 to integrate $\omega_{A}$ to a group cocycle, $\int \omega_{A}$, on $N_{A}$.

First, however, we must pause to give some preliminary definitions concerning calculus on $N_{A}$, which is diffeomorphic to the $A_{0}$-module $\mathfrak{n}_{A}$. Recall from Section 5.1 that a map

$$
\varphi: V \rightarrow W
$$

between two $A_{0}$-modules said to be $\boldsymbol{A}_{\mathbf{0}}$-smooth if it is smooth in the ordinary sense and its derivative

$$
\varphi_{*}: T_{x} V \rightarrow T_{\varphi(x)} W
$$


is $A_{0}$-linear at each point $x \in V$. Here, the $A_{0}$-module structure on each tangent space comes from the canonical identification with the ambient vector space:

$$
T_{x} V \cong V, \quad T_{\varphi(x)} W \cong W .
$$

It is clear that the identity is $A_{0}$-smooth and the composite of any two $A_{0}$-smooth maps is $A_{0}$-smooth. A vector field $X$ on $V$ is $\boldsymbol{A}_{0}$-smooth if $X f$ is an $A_{0}$-smooth function for all $f: V \rightarrow A_{0}$ that are $A_{0}$-smooth. An $A_{0}$-valued differential $p$-form $\omega$ on $V$ is $\boldsymbol{A}_{\mathbf{0}}$-smooth if $\omega\left(X_{1}, \ldots, X_{p}\right)$ is an $A_{0}$-smooth function for all $A_{0}$-smooth vector fields $X_{1}, \ldots, X_{p}$.

Now, we return to integrating $\omega$. As a first step, because $\mathfrak{n}_{A}=T_{1} N_{A}$, we can view $\omega_{A}$ as an $A_{0}$-valued $p$-form on $T_{1} N_{A}$. Using left translation, we can extend this to a left-invariant $A_{0}$-valued $p$-form on $N_{A}$. Indeed, we can do this for any $A_{0}$-valued $p$-cochain on $\mathfrak{n}_{A}$ :

$$
C^{p}\left(\mathfrak{n}_{A}\right) \cong\left\{\text { left-invariant } A_{0} \text {-valued } p \text {-forms on } N_{A}\right\} \text {. }
$$

Note that any left-invariant $A_{0^{-}}$-valued form on $N_{A}$ is automatically $A_{0^{-}}$ smooth: multiplication on $N_{A}$ is given by the $A_{0}$-smooth operation induced from multiplication on $N$, and so left translation on $N_{A}$ is $A_{0}$-smooth. We can differentiate and integrate $A_{0}$-valued $p$-forms in just the same way as we would real-valued $p$-forms, and the de Rham differential $d$ of left-invariant $p$-forms coincides with the usual differential of Lie algebra $p$-cochains.

As before, we need a notion of simplices in $N$. Since $N$ is a supermanifold, the vertices of a simplex should not be points of $N$, but rather $A$-points for arbitrary Grassmann algebras $A$. This means that for any $(p+1)$-tuple of $A$-points, we want to get a $p$-simplex:

$$
\left[n_{0}, n_{1}, \ldots, n_{p}\right]: \Delta^{p} \rightarrow N_{A},
$$

where, once again, $\Delta^{p}$ is the standard $p$-simplex in $\mathbb{R}^{p+1}$, and this map is required to be smooth. But this only defines a $p$-simplex in $N_{A}$. To really get our hands on a $p$-simplex in $N$, we need it to depend functorially on the choice of Grassmann algebra $A$ we use to probe $N$. So if $f: A \rightarrow B$ is a homomorphism between Grassmann algebras and $N_{f}: N_{A} \rightarrow N_{B}$ is the induced map between $A$-points and $B$-points, we require:

$$
N_{f} \circ\left[n_{0}, n_{1}, \ldots, n_{p}\right]=\left[N_{f}\left(n_{0}\right), N_{f}\left(n_{1}\right), \ldots, N_{f}\left(n_{p}\right)\right]
$$

Thus given a collection of maps:

$$
\left(\varphi_{p}\right)_{A}: \Delta^{p} \times\left(N_{A}\right)^{p+1} \rightarrow N_{A}
$$


"ATMP-16-5-A4-HUE" — 2013/4/30 — 17:32 — page 1564 — \#80

for all $A$ and $p \geq 0$, we say this collection defines a left-invariant notion of simplices in $N$ if

- each $\left(\varphi_{p}\right)_{A}$ is smooth, and for each $x \in \Delta^{p}$, the restriction:

$$
\left(\varphi_{p}\right)_{A}:\{x\} \times N_{A}^{p+1} \rightarrow N_{A}
$$

is $A_{0}$-smooth;

- it defines a left-invariant notion of simplices in $N_{A}$ for each $A$, as in Definition 12;

- the following diagram commutes for all homomorphisms $f: A \rightarrow B$ :

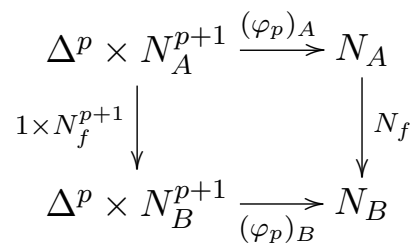

We can use a left-invariant notion of simplices to define a cochain map $\int: C^{\bullet}(\mathfrak{n}) \rightarrow C^{\bullet}(N):$

Proposition 24. Let $\mathfrak{n}$ be a nilpotent Lie superalgebra, and let $N$ be the exponential supergroup which integrates $\mathfrak{n}$. If $N$ is equipped with a leftinvariant notion of simplices, then there is a cochain map:

$$
\int: C_{0}^{\bullet}(\mathfrak{n}) \rightarrow C^{\bullet}(N)
$$

which sends the even Lie superalgebra p-cochain $\omega$ to the supergroup p-cochain $\int \omega$, given on A-points by:

$$
\left(\int \omega\right)_{A}\left(n_{1}, \ldots, n_{p}\right)=\int_{\left[1, n_{1}, n_{1} n_{2}, \ldots, n_{1} n_{2} \ldots n_{p}\right]} \omega_{A}
$$

for $n_{1}, \ldots, n_{p} \in N_{A}$.

Proof. First, we must check that $\int \omega_{A}: N_{A}^{p} \rightarrow A_{0}$ is natural in $A$ and $A_{0^{-}}$ smooth, and hence defines a map of supermanifolds:

$$
\int \omega: N^{p} \rightarrow \mathbb{R}
$$

Smoothness is clear, so we check naturality and the $A_{0}$-linearity of the derivative. 
To check naturality, let $f: A \rightarrow B$ be a homomorphism, and $N_{f}: N_{A} \rightarrow$ $N_{B}$ be the induced map from $A$-points to $B$-points. We wish to show the following square commutes:

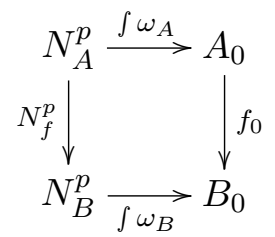

For $A$-points $n_{1}, \ldots, n_{p} \in N_{A}$, we have:

$$
f_{0} \int_{\left[1, n_{1}, n_{1} n_{2}, \ldots, n_{1} n_{2} \ldots n_{p}\right]} \omega_{A}=\int_{\left[1, n_{1}, n_{1} n_{2}, \ldots, n_{1} \ldots n_{p}\right]} f_{0} \omega_{A} .
$$

Since $\omega_{A}: \Lambda^{p} \mathfrak{n}_{A} \rightarrow A_{0}$ is natural itself, we have:

$$
f_{0} \omega_{A}\left(X_{1}, \ldots, X_{p}\right)=\omega_{B}\left(\mathfrak{n}_{f}\left(X_{1}\right), \ldots, \mathfrak{n}_{f}\left(X_{p}\right)\right)
$$

for all $X_{1}, \ldots, X_{p} \in \mathfrak{n}_{A}$. Now, under the identification $\mathfrak{n}_{A} \cong T_{1} N_{A}$, the linear map:

$$
\mathfrak{n}_{f}: \mathfrak{n}_{A} \rightarrow \mathfrak{n}_{B}
$$

is the derivative of the map $N_{f}: N_{A} \rightarrow N_{B}$, so we get the pullback of $\omega_{A}$ along $N_{f}$ :

$$
\begin{aligned}
\omega_{B}\left(\mathfrak{n}_{f}\left(X_{1}\right), \ldots, \mathfrak{n}_{f}\left(X_{p}\right)\right) & =\omega_{B}\left(\left(N_{f}\right)_{*}\left(X_{1}\right), \ldots,\left(N_{f}\right)_{*}\left(X_{p}\right)\right) \\
& =N_{f}^{*} \omega_{B}\left(X_{1}, \ldots, X_{p}\right) .
\end{aligned}
$$

Finally:

$$
\begin{aligned}
f_{0} \int_{\left[1, n_{1}, n_{1} n_{2}, \ldots, n_{1} n_{2} \ldots n_{p}\right]} \omega_{A} & =\int_{\left[1, n_{1}, n_{1} n_{2}, \ldots, n_{1} n_{2} \ldots n_{p}\right]} N_{f}^{*} \omega_{B} \\
& =\int_{N_{f} \circ\left[1, n_{1}, n_{1} n_{2}, \ldots, n_{1} n_{2} \ldots n_{p}\right]} \omega_{B} \\
& =\int_{\left[1, N_{f}\left(n_{1}\right), N_{f}\left(n_{1}\right) N_{f}\left(n_{2}\right), \ldots, N_{f}\left(n_{1}\right) N_{f}\left(n_{2}\right) \ldots N_{f}\left(n_{p}\right)\right]} \omega_{B}
\end{aligned}
$$

where in the last step we have used the fact that $\left[1, n_{1}, n_{1} n_{2}, \ldots, n_{1} \ldots n_{p}\right]$ is a left-invariant simplex in $N$, as well as the fact that $N_{f}$ is a group homomorphism. But this says exactly that $\int \omega_{A}$ is natural in $A$. 
Next, we check that $\int \omega_{A}$ has a derivative that is $A_{0}$-linear. Briefly, this holds because the derivative of $\left(\varphi_{p}\right)_{A}: \Delta^{p} \times N_{A}^{p+1} \rightarrow N_{A}$ is $A_{0}$-linear on each factor of $N_{A}$. The $A_{0}$-linearity of the derivative of $\int \omega_{A}$ then follows from the elementary analytic fact that integration with respect to one variable and differentiation with respect to another commute with each other, at least when the integration is performed over a compact set. For a complete calculation, see the proof of Proposition 10.2 in Huerta [33].

Thus, $\int \omega: N_{A}^{p} \rightarrow A_{0}$, being natural in $A$ and $A_{0}$-smooth, defines a map of supermanifolds $\int \omega: N^{p} \rightarrow \mathbb{R}$. We therefore have a map:

$$
\int: C_{0}^{\bullet}(\mathfrak{n}) \rightarrow C^{\bullet}(N) \text {. }
$$

It remains check that it is a cochain map. Indeed, $\int \omega_{A}$ is the composite of the cochain maps for each $A$ :

$$
\omega \mapsto \omega_{A} \mapsto \int \omega_{A},
$$

and it follows that $\int$ is a cochain map.

Finally, we shall prove that there is a left-invariant notion of simplices with which we can equip $N$. For a fixed Grassmann algebra $A$, the Lie group $N_{A}$ is exponential. We shall show that if we take:

$$
\left(\varphi_{p}\right)_{A}: \Delta^{p} \times N_{A}^{p+1} \rightarrow N_{A}
$$

to be the standard notion of left-invariant simplices in Proposition 14, then this defines a left-invariant notion of simplices in $N$. The key is to note that each stage of the inductive definition of $\left(\varphi_{p}\right)_{A}$ we get maps that are natural in $A$.

Proposition 25. Let $N$ be the exponential supergroup of the nilpotent Lie superalgebra $\mathfrak{n}$. Fix a smoothing factor $\ell:[0,1] \rightarrow[0,1]$. For each Grassmann algebra $A$ and $p \geq 0$, define:

$$
\left(\varphi_{p}\right)_{A}: \Delta^{p} \times N_{A}^{p+1} \rightarrow N_{A}
$$

to be the standard left-invariant notion of simplices with smoothing factor $\ell$. Then this defines a left-invariant notion of simplices in $N$.

Proof. Fix Grassmann algebras $A$ and $B$ and a homomorphism $f: A \rightarrow B$. We proceed by induction on $p$. For $p=0$, the maps:

$$
\begin{aligned}
& \left(\varphi_{0}\right)_{A}: \Delta^{0} \times N_{A} \rightarrow N_{A}, \\
& \left(\varphi_{0}\right)_{B}: \Delta^{0} \times N_{B} \rightarrow N_{B},
\end{aligned}
$$


are the obvious projections. The fact that:

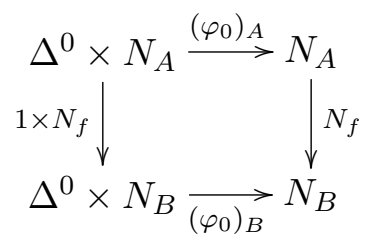

commutes is then automatic.

For arbitrary $p$, suppose that the following square commutes:

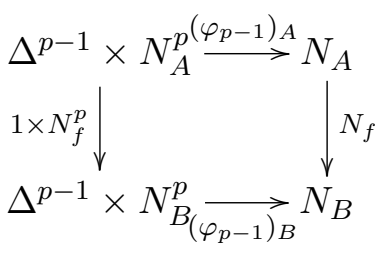

and that $\left(\varphi_{p-1}\right)_{A}$ and $\left(\varphi_{p-1}\right)_{B}$ are $A_{0^{-}}$and $B_{0^{-}}$-smooth. In other words, the above square says that for any $p$-tuple of $A$-points, we have:

$$
N_{f} \circ\left[n_{1}, \ldots, n_{p}\right]=\left[N_{f}\left(n_{1}\right), \ldots, N_{f}\left(n_{p}\right)\right] .
$$

We construct $\left(\varphi_{p}\right)_{A}$ and $\left(\varphi_{p}\right)_{B}$ from $\left(\varphi_{p-1}\right)_{A}$ and $\left(\varphi_{p-1}\right)_{B}$, respectively, using the apex-base construction. That is, given the $(p-1)$-simplex $\left[n_{1}, \ldots\right.$, $\left.n_{p}\right]$ given by $\left(\varphi_{p-1}\right)_{A}$ for the $A$-points $n_{1}, \ldots, n_{p} \in N_{A}$, we define the based p-simplex:

$$
\left[1, n_{1}, \ldots, n_{p}\right]
$$

in $N_{A}$ by using the exponential map $\exp _{A}$ to sweep out a path from the apex 1 to each point of the base $\left[n_{1}, \ldots, n_{p}\right]$. Similarly, we define the based -simplex:

$$
\left[1, N_{f}\left(n_{1}\right), \ldots, N_{f}\left(n_{p}\right)\right]
$$

in $N_{B}$ by using the exponential map $\exp _{B}$ to sweep out a path from the apex 1 to each point of the base $\left[N_{f}\left(n_{1}\right), \ldots, N_{f}\left(n_{p}\right)\right]$. From the naturality of exp, we will establish that:

$$
N_{f} \circ\left[1, n_{1}, \ldots, n_{p}\right]=\left[1, N_{f}\left(n_{1}\right), \ldots, N_{f}\left(n_{p}\right)\right] .
$$

To verify this claim, let

$$
\exp _{A}(X)=\left[n_{1}, \ldots, n_{p}\right](x), \text { for some } x \in \Delta^{p-1}
$$


be a point of the base in $N_{A}$. By the inductive hypothesis, $N_{f}\left(\exp _{A}(X)\right)=$ $\exp _{B}\left(\mathfrak{n}_{f}(X)\right)$ is the corresponding point of the base in $N_{B}$. We wish to see that points of the path $\exp _{A}(\ell(t) X)$ connecting 1 to $\exp _{A}(X)$ in $N_{A}$ correspond via $N_{f}$ to points on the path $\exp _{B}\left(\ell(t) \mathfrak{n}_{f}(X)\right)$ connecting 1 to $\exp _{B}\left(\mathfrak{n}_{f}(X)\right)$ in $N_{B}$. But this is automatic, because:

$$
N_{f}\left(\exp _{A}(\ell(t) X)=\exp _{B}\left(\mathfrak{n}_{f}(\ell(t) X)\right)=\exp _{B}\left(\ell(t) \mathfrak{n}_{f}(X)\right),\right.
$$

where in the last step we use the fact that $\mathfrak{n}_{f}: \mathfrak{n}_{A} \rightarrow \mathfrak{n}_{B}$ is linear. Thus, it is true that:

$$
N_{f} \circ\left[1, n_{1}, \ldots, n_{p}\right]=\left[1, N_{f}\left(n_{1}\right), \ldots, N_{f}\left(n_{p}\right)\right],
$$

for based $p$-simplices.

Using left translation, we can show that:

$$
N_{f} \circ\left[n_{0}, n_{1}, \ldots, n_{p}\right]=\left[N_{f}\left(n_{0}\right), N_{f}\left(n_{1}\right), \ldots, N_{f}\left(n_{p}\right)\right] .
$$

for all $p$-simplices. In other words, the following diagram commutes:

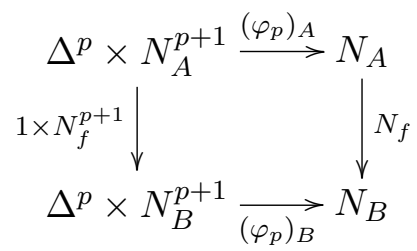

Because each step in the apex-base construction preserves $A_{0}$ - or $B_{0}$-smoothness, we note that $\left(\varphi_{p}\right)_{A}$ and $\left(\varphi_{p}\right)_{B}$ are $A_{0^{-}}$and $B_{0^{-}}$-smooth, respectively. The result now follows for all $p$ by induction.

\section{Superstring Lie 2-supergroups}

We are now ready to unveil the Lie 2-supergroup that integrates our favorite Lie 2-superalgebra, $\mathfrak{s u p e r s t r i n g}(n+1,1)$. Remember, this is the Lie 2-superalgebra which occurs only in the dimensions for which string theory makes sense: $n+2=3,4,6$ and 10 . It is not nilpotent, since the Poincaré superalgebra $\mathfrak{s i s o}(n+1,1)$ in degree 0 of $\mathfrak{s u p e r s t r i n g}(n+1,1)$ is not nilpotent. Nonetheless, we are equipped to integrate it using only the tools we have built to perform this task for nilpotent Lie $n$-superalgebras. 
The road to this result has been a long one, and there is yet some ground to cover before we are finished. So, let us take stock of our progress before we move ahead:

- In spacetime dimensions $n+2=3,4,6$ and 10, we used division algebras to construct a 3 -cocycle $\alpha$ on the supertranslation algebra:

$$
\mathcal{T}=V \oplus S
$$

which is nonzero only when it eats a vector and two spinors:

$$
\alpha(A, \psi, \phi)=\langle\psi, A \phi\rangle .
$$

- Because $\alpha$ is invariant under the action of $\mathfrak{s o}(n+1,1)$, it can be extended to a 3-cocycle on the Poincaré superalgebra:

$$
\mathfrak{s i s o}(n+1,1)=\mathfrak{s o}(n+1,1) \ltimes \mathcal{T} .
$$

The extension is just defined to vanish outside of $\mathcal{T}$, and we call it $\alpha$ as well.

- Therefore, in spacetime dimensions $n+2$, we get a Lie 2-superalgebra $\mathfrak{s u p e r s t r i n g}(n+1,1)$ by extending $\mathfrak{s i s o}(n+1,1)$ by the 3 -cocycle $\alpha$.

In the last section, we built the technology necessary to integrate Lie superalgebra cocycles to supergroup cocycles, provided the Lie superalgebra in question is nilpotent. This allows us to integrate nilpotent Lie $n$-superalgebras to $n$-supergroups. But $\mathfrak{s u p e r s t r i n g}(n+1,1)$ is not nilpotent, so we cannot use this directly here.

However, the cocycle $\alpha$ is supported on a nilpotent subalgebra: the supertranslation algebra, $\mathcal{T}$. This saves the day: we can integrate $\alpha$ as a cocycle on $\mathcal{T}$. This gives us a cocycle $\int \alpha$ supertranslation supergroup, $T$. We will then be able to extend this cocycle to the Poincaré supergroup, thanks to its invariance under Lorentz transformations.

The following proposition helps us to accomplish this, but takes its most beautiful form when we work with "homogeneous supergroup cochains", which we have not actually defined. Rest assured - they are exactly what you expect. If $G$ is a supergroup that acts on the abelian supergroup $M$ by automorphism, a homogeneous $\boldsymbol{M}$-valued $\boldsymbol{p}$-cochain on $G$ is a smooth map:

$$
F: G^{p+1} \rightarrow M
$$

such that, for any Grassmann algebra $A$ and $A$-points $g, g_{0}, \ldots, g_{p} \in G_{A}$ :

$$
F_{A}\left(g g_{0}, g g_{1}, \ldots, g g_{p}\right)=g F_{A}\left(g_{1}, \ldots, g_{p}\right) .
$$


We can define the supergroup cohomology of $G$ using homogeneous or inhomogeneous cochains, just as was the case with Lie group cohomology.

Proposition 26. Let $G$ and $H$ be Lie supergroups such that $G$ acts on $H$, and let $M$ be an abelian supergroup on which $G \ltimes H$ acts by automorphism. Given a homogeneous $M$-valued $p$-cochain $F$ on $H$ :

$$
F: H^{p+1} \rightarrow M
$$

we can extend it to a map of supermanifolds:

$$
\tilde{F}:(G \ltimes H)^{p+1} \rightarrow M
$$

by pulling back along the projection $(G \ltimes H)^{p+1} \rightarrow H^{p+1}$. In terms of A-points

$$
\left(g_{0}, h_{0}\right), \ldots,\left(g_{p}, h_{p}\right) \in G_{A} \ltimes H_{A},
$$

this means $\tilde{F}$ is defined by:

$$
\tilde{F}_{A}\left(\left(g_{0}, h_{0}\right), \ldots,\left(g_{p}, h_{p}\right)\right)=F_{A}\left(h_{0}, \ldots, h_{p}\right),
$$

Then $\tilde{F}$ is a homogeneous p-cochain on $G \ltimes H$ if and only if $F$ is G-equivariant, and in this case $d \tilde{F}=\widetilde{d F}$.

Proof. We work over $A$-points, $G_{A} \ltimes H_{A}$. Denoting the action of $g \in G_{A}$ on $h \in H_{A}$ by $h^{g}$, recall that multiplication in the semidirect product $G_{A} \ltimes H_{A}$ is given by:

$$
\left(g_{1}, h_{1}\right)\left(g_{2}, h_{2}\right)=\left(g_{1} g_{2}, h_{1} h_{2}^{g_{1}}\right) .
$$

Now suppose $\tilde{F}$ is homogeneous. By definition of homogeneity, we have:

$$
\tilde{F}_{A}\left((g, h)\left(g_{0}, h_{0}\right), \ldots,(g, h)\left(g_{p}, h_{p}\right)\right)=(g, h) \tilde{F}_{A}\left(\left(g_{0}, h_{0}\right), \ldots,\left(g_{p}, h_{p}\right)\right) .
$$

Multiplying out each pair on the left and using the definition of $\tilde{F}$ on both sides, we get:

$$
F_{A}\left(h h_{0}^{g}, \ldots, h h_{p}^{g}\right)=(g, h) F_{A}\left(h_{0}, \ldots, h_{p}\right)
$$


Writing $(g, h)$ as $(1, h)(g, 1)$, and pulling $h$ out on the left-hand side, we now obtain:

$$
(1, h) F_{A}\left(h_{0}^{g}, \ldots, h_{p}^{g}\right)=(1, h)(g, 1) F_{A}\left(h_{0}, \ldots, h_{p}\right)
$$

Cancelling $(1, h)$ from both sides, this last equation just says that $F_{A}$ is $G_{A^{-}}$ equivariant. The converse follows from reversing this calculation. Since this holds for any Grassmann algebra $A$, we conclude that $\tilde{F}$ is homogeneous if and only if $F$ is $G$-equivariant.

When $F$ is $G$-equivariant, it is easy to see that $d F$ is also, and that $d \tilde{F}=\widetilde{d F}$, so we are done.

Now, at long last, we are ready to integrate $\alpha$. In the following proposition, $T$ denotes the supertranslation group, the exponential supergroup of the supertranslation algebra $\mathcal{T}$.

Proposition 27. In dimensions $n+2=3,4,6$ and 10, the Lie supergroup 3-cocycle $\int \alpha$ on the supertranslation group $T$ is invariant under the action of $\operatorname{Spin}(n+1,1)$.

This is an immediate consequence of the following:

Proposition 28. Let $H$ be the exponential supergroup of a nilpotent Lie superalgebra $\mathfrak{h}$. Assume $H$ is equipped with its standard left-invariant notion of simplices, and let $G$ be a Lie supergroup that acts on $H$ by automorphism. If $\omega \in C_{0}^{p}(\mathfrak{h})$ is an even Lie superalgebra $p$-cochain which is invariant under the induced action of $G$ on $\mathfrak{h}$, then $\int \omega \in C^{p}(H)$ is a Lie supergroup $p$ cochain, which is invariant under the action of $G$ on $H$.

Proof. Fixing a Grassmann algebra $A$, we must prove that

$$
\int_{\left[h_{0}^{g}, h_{1}^{g}, \ldots, h_{p}^{g}\right]} \omega_{A}=\int_{\left[h_{0}, h_{1}, \ldots, h_{p}\right]} \omega_{A},
$$

for all $A$-points $g \in G_{A}$ and $h_{0}, h_{1}, \ldots, h_{p} \in H_{A}$. We shall see this follows from the fact that the $p$-simplices in $H$ are themselves $G$-equivariant, in the sense that:

$$
\left[h_{0}^{g}, h_{1}^{g}, \ldots, h_{p}^{g}\right]=\left[h_{0}, h_{1}, \ldots, h_{p}\right]^{g} .
$$


Assuming this for the moment, let us check that our result follows. Indeed, applying the above equation, we get:

$$
\begin{aligned}
\int_{\left[h_{0}^{g}, h_{1}^{g}, \ldots, h_{p}^{g}\right]} \omega_{A} & =\int_{\left[h_{0}, h_{1}, \ldots, h_{p}\right]^{g}} \omega_{A} \\
& =\int_{\left[h_{0}, h_{1}, \ldots, h_{p}\right]} \operatorname{Ad}(g)^{*} \omega_{A} \\
& =\int_{\left[h_{0}, h_{1}, \ldots, h_{p}\right]} \omega_{A},
\end{aligned}
$$

where the final step uses $\operatorname{Ad}(g)^{*} \omega_{A}=\omega_{A}$, which is just the $G$-invariance of $\omega$.

It therefore remains to prove the equation $\left[h_{0}^{g}, h_{1}^{g}, \ldots, h_{p}^{g}\right]=\left[h_{0}, h_{1}, \ldots\right.$, $\left.h_{p}\right]^{g}$ actually holds. Note that this is the same as saying that the map

$$
\left(\varphi_{p}\right)_{A}: \Delta^{p} \times H_{A}^{p+1} \rightarrow H_{A}
$$

is $G_{A^{-}}$equivariant. We check it by induction on $p$.

For $p=0$, the map:

$$
\left(\varphi_{0}\right)_{A}: \Delta^{0} \times H_{A} \rightarrow H_{A}
$$

is just the projection, and $G_{A}$-equivariance is obvious. So fix some $p \geq 0$ and suppose that $\left(\varphi_{p-1}\right)_{A}$ is $G_{A}$-equivariant. We now construct $\left(\varphi_{p}\right)_{A}$ from $\left(\varphi_{p-1}\right)_{A}$ using the apex-base construction, and show that equivariance is preserved.

So, given the $(p-1)$-simplex $\left[h_{1}, \ldots, h_{p}\right]$ given by $\left(\varphi_{p-1}\right)_{A}$ for the $A$-points $h_{1}, \ldots, h_{p} \in H_{A}$, we define the based $p$-simplex:

$$
\left[1, h_{1}, \ldots, h_{p}\right]
$$

in $H_{A}$ by using the exponential map to sweep out a path from the apex 1 to each point of the base $\left[h_{1}, \ldots, h_{p}\right]$. In a similar way, we define the based -simplex:

$$
\left[1, h_{1}^{g}, \ldots, h_{p}^{g}\right]
$$

By hypothesis, $\left[h_{1}^{g}, \ldots, h_{p}^{g}\right]=\left[h_{1}, \ldots, h_{p}\right]^{g}$, and since the exponential map $\exp : \mathfrak{h}_{A} \rightarrow H_{A}$ is itself $G_{A}$-equivariant, it follows for based $p$-simplices that:

$$
\left[1, h_{1}^{g}, \ldots, h_{p}^{g}\right]=\left[1, h_{1}, \ldots, h_{p}\right]^{g} .
$$


“ATMP-16-5-A4-HUE" — 2013/4/30 — 17:32 — page 1573 — \#89

The result now follows for all $p$-simplices by left translation. This completes the proof.

Because $\alpha$ is $\operatorname{Spin}(n+1,1)$-invariant, it follows from Propostion 28 that in dimensions $3,4,6$ and 10, the cocycle $\int \alpha$ on the supertranslations can be extended to a 3 -cocycle on the full Poincaré supergroup:

$$
\operatorname{SISO}(n+1,1)=\operatorname{Spin}(n+1,1) \ltimes T,
$$

By a slight abuse of notation, we continue to denote this extension by $\int \alpha$. As an immediate consequence, we have:

Theorem 29. In dimensions $n+2=3,4,6$ and 10, there exists a slim Lie 2-supergroup formed by extending the Poincaré supergroup $\operatorname{SISO}(n+1,1)$ by the 3-cocycle $\int \alpha$, which we call we the superstring Lie 2-supergroup, Superstring $(n+1,1)$.

\section{Acknowledgements}

We thank Jim Dolan, Ezra Getzler, Hisham Sati and Christoph Wockel for helpful conversations. We especially thank Urs Schreiber for reviewing this manuscript and freely sharing his insight into higher gauge theory. Finally, we thank John Baez for his untiring help as he supervised the thesis on which this paper is based. This work was partially supported by FQXi grant RFP2-08-04 and the Australian Research Council's Discovery Projects funding scheme (project number DP110100072).

\section{Appendix A Explicitly integrating 0-, 1-, 2- and 3-cochains}

In this appendix, in order to get a feel for the integration procedure given in Proposition 13, we shall explicitly calculate some Lie group cochains from Lie algebra cochains. The resulting formulas are polynomials on the Lie group, at least in the nilpotent case. It is important to note, however, that we did not need these explicit formulas anywhere in this paper. It was enough to understand that they exist, and have the properties described in Section 4.1. We nonetheless suspect that explicit formulas will prove useful in future work, so we collect some here.

To facilitate this calculation, we shall also have to explicitly construct some low-dimensional left-invariant simplices. For 0-cochains and 1-cochains, we will find the task very easy — we only need our Lie group $G$ to be exponential. On the other hand, for 2 - and 3-cochains, the construction gets much 
harder. This complexity shows just how powerful the abstract approach of Section 4.1 actually is - imagine having to prove Proposition 13 through an explicit integration such as those we present here!

So, for 2- and 3-cochains, we simplify the problem by assuming our Lie algebra $\mathfrak{g}$ to be 2-step nilpotent: all brackets of brackets are zero. This allows us to use a simplified form of the Baker-Campbell-Hausdorff formula:

$$
\exp (X) \exp (Y)=\exp \left(X+Y+\frac{1}{2}[X, Y]\right)
$$

and the Zassenhaus formula:

$$
\begin{aligned}
\exp (X+Y) & =\exp (X) \exp (Y) \exp \left(-\frac{1}{2}[X, Y]\right) \\
& =\exp (X) \exp \left(Y-\frac{1}{2}[X, Y]\right)
\end{aligned}
$$

Partially, this nilpotentcy assumption just makes our calculations tenable, but secretly it is because our main application for these ideas is to 2-step nilpotent Lie superalgebras.

\section{0-cochains}

Let $\omega$ be a Lie algebra 0 -cochain: that is, a real number. Then $\int \omega=\omega$ is a Lie group 0 -cochain. We can view it as the integral of $\omega$ over the 0 -simplex [1].

\section{1-cochains}

Let $\omega$ be a Lie algebra 1-cochain: that is, a linear map

$$
\omega: \mathfrak{g} \rightarrow \mathbb{R},
$$

which we extend to a 1 -form on $G$ by left translation. We define a Lie group 1 -cochain $\int \omega$ by integrating $\omega$ over 1 -simplices in $G$. In particular,

$$
\int \omega(g)=\int_{[1, g]} \omega .
$$

Since $G$ is exponential, it has a standard left-invariant notion of 1-simplex, given by exponentiation. So, if $g=\exp (X)$, then the 1 -simplex $[1, g]$ is 
given by

$$
[1, g](t)=\exp (t X), \quad 0 \leq t \leq 1
$$

We denote this map by $\varphi$ for brevity. So:

$$
\int \omega(g)=\int_{0}^{1} \omega(\dot{\varphi}(t)) d t
$$

Noting that the derivative of $\varphi$ is

$$
\dot{\varphi}(t)=\exp (t X) X
$$

we have

$$
\int \omega(g)=\int_{0}^{1} \omega(\exp (t X) X) d t=\int_{0}^{1} \omega(X) d t=\omega(X),
$$

where we have used the left invariance of $\omega$. In summary,

$$
\int \omega(g)=\omega(X),
$$

for $g=\exp (X)$.

As a check on this, note that because we have proved $\int$ is a cochain map, $\int \omega$ should be a cocycle whenever $\omega$ is. So let us verify this. Assume $d \omega=0$. That is, for all $X$ and $Y \in \mathfrak{g}$, we have:

$$
d \omega(X, Y)=-\omega([X, Y])=0 .
$$

So the cocycle condition merely says that $\omega$ must vanish on brackets.

Now compute the coboundary of $\int \omega$. As a function of a single group element, $\int \omega$ is an inhomogeneous Lie group 2-cochain. Hence, we must use the coboundary formula from Section 2, which we recall here for convenience: when $f: G^{p} \rightarrow \mathbb{R}$ is an inhomogeneous Lie group $p$-cochain, its coboundary is:

$$
\begin{aligned}
& d f\left(g_{1}, \ldots, g_{p+1}\right) \\
& \quad=\sum_{i=1}^{p}(-1)^{i} f\left(g_{1}, \ldots, g_{i-1}, g_{i} g_{i+1}, g_{i+2}, \ldots, g_{p+1}\right) \\
& \quad+(-1)^{p+1} f\left(g_{1}, \ldots, g_{p}\right) .
\end{aligned}
$$


In the case of $\int \omega$, this becomes simply:

$$
d \int \omega(g, h)=\int \omega(h)-\int \omega(g h)+\int \omega(g) .
$$

Finally, check that this coboundary is zero when $\omega$ is a cocycle, and hence that $\int \omega$ is a cocycle whenever $\omega$ is. If $g=\exp (X)$ and $h=\exp (Y)$, we have

$$
g h=\exp (X) \exp (Y)=\exp \left(X+Y+\frac{1}{2}[X, Y]+\cdots\right)
$$

by the Baker-Campbell-Hausdorff formula, and thus:

$$
d \int \omega(g, h)=\omega(Y)-\omega\left(X+Y+\frac{1}{2}[X, Y]+\cdots\right)+\omega(X)=0
$$

where we have used $\omega$ 's linearity along with the cocycle condition that $\omega$ must vanish on brackets.

\section{2-cochains}

As we have just seen, 0-cochains and 1-cochains are easily integrated on any exponential Lie group, and the result is always a polynomial Lie group cochain. Unfortunately, even for 2-cochains, the integration is much more complicated, and no longer polynomial unless $\mathfrak{g}$ is nilpotent. So, at this point, we will simplify matters by assuming $\mathfrak{g}$ to be 2 -step nilpotent. To hint at this with our notation, we will now call our Lie algebra $\mathfrak{n}$ and the corresponding simply-connected Lie group $N$.

Let $\omega$ be a Lie algebra 2-cochain: that is, a left-invariant 2-form. We define a Lie group 2 -cochain $\int \omega$ by integrating $\omega$ over 2 -simplices in $N$. In particular:

$$
\int \omega(g, h)=\int_{[1, g, g h]} \omega
$$

Now suppose $g=\exp (X)$ and $h=\exp (Y)$. Recall we that obtain the 2 -simplex $[1, g, g h]$ using the apex-base construction: we connect each point of the base $[g, g h]=g[1, h]$ to 1 by the exponential map. Since $[1, h](t)=$ $\exp (t Y)$, the base is parameterized by

$$
[g, g h](t)=g \exp (t Y)=\exp \left(X+t Y+\frac{t}{2}[X, Y]\right)
$$


by the Baker-Campbell-Hausdorff formula. Now let us construct $[1, g, g h]$ by first constructing a map from the square

$$
\varphi:[0,1] \times[0,1] \rightarrow N
$$

given by

$$
\varphi(s, t)=\exp \left(s\left(X+t Y+\frac{t}{2}[X, Y]\right)\right)
$$

At this stage in our general construction, since this map is 1 on the $\{0\} \times$ $[0,1]$ edge of the square, we would typically quotient the square out by this edge to obtain a map from the standard 2-simplex. However in practice, we do not need to do this. Since the integral $\int_{[1, g, g h]} \omega$ is invariant under reparameterization, we might as well parameterize our 2 -simplex $[1, g, g h]$ with $\varphi$ and integrate over the square to obtain:

$$
\int \omega(g, h)=\int_{0}^{1} \int_{0}^{1} \omega\left(\frac{\partial \varphi}{\partial s}, \frac{\partial \varphi}{\partial t}\right) d s d t .
$$

Our task has essentially been reduced to computing the partial derivatives of $\varphi$. Thanks to the left invariance of $\omega$, we may as well left translate these partials back to 1 once we have them, since:

$$
\omega\left(\frac{\partial \varphi}{\partial s}, \frac{\partial \varphi}{\partial t}\right)=\omega\left(\varphi^{-1} \frac{\partial \varphi}{\partial s}, \varphi^{-1} \frac{\partial \varphi}{\partial t}\right)
$$

Let us begin with $\frac{\partial \varphi}{\partial s}$. Since the exponent of $\varphi(s, t)=\exp (s(X+t Y+$ $\left.\left.\frac{t}{2}[X, Y]\right)\right)$ is linear in $s$, this is simply:

$$
\frac{\partial \varphi}{\varphi s}(s, t)=\varphi(s, t)\left(X+t Y \frac{t}{2}[X, Y]\right)
$$

This is a tangent vector at $\varphi(s, t)$. We can left translate it back to 1 to obtain:

$$
\left.\varphi^{-1} \frac{\partial \varphi}{\partial s}=X+t Y \frac{t}{2}[X, Y]\right) .
$$

The partial with respect to $t$ is slightly harder, because the exponent is not linear in $t$. To compute this, we need the Zassenhaus formula, Formula 
3 , to separate the terms linear in $t$ from those that are not. Applying this, we obtain

$$
\varphi(s, t)=\exp (s X) \exp \left(s t Y+\frac{s t}{2}[X, Y]-\frac{s^{2} t}{2}[X, Y]\right) .
$$

Differentiating this with respect to $t$ and left translating the result to 1 , we get:

$$
\varphi^{-1} \frac{\partial \varphi}{\partial t}=s Y+\frac{s-s^{2}}{2}[X, Y]
$$

Substituting these partial derivatives into the integral, our problem becomes:

$$
\int \omega(g, h)=\int_{0}^{1} \int_{0}^{1} \omega\left(X+t Y+\frac{t}{2}[X, Y], s Y+\frac{s-s^{2}}{2}[X, Y]\right) d s d t .
$$

It is now easy enough, using $\omega$ 's bilinearity and antisymmetry, to bring all the polynomial coefficients out and integrate them, obtaining an expression which is the sum of three terms:

$$
\int \omega(g, h)=\frac{1}{2} \omega(X, Y)+\frac{1}{12} \omega(X,[X, Y])-\frac{1}{12} \omega(Y,[X, Y]) .
$$

Nevertheless, we would like to do this calculation explicitly. In essence, we use $\omega$ 's bilinearity and antisymmetry to our advantage, to write these coefficients as integrals of various determinants. To wit, the coefficient of $\omega(X, Y)$ is the integral of the determinant

$$
\left|\begin{array}{ll}
1 & t \\
0 & s
\end{array}\right|=s
$$

which we obtain from reading off the coefficients of $X$ and $Y$ in the integrand:

$$
\omega\left(X+t Y+\frac{t}{2}[X, Y], s Y+\frac{s-s^{2}}{2}[X, Y]\right)
$$

So the coefficient of $\omega(X, Y)$ is $\int_{0}^{1} \int_{0}^{1} s d s d t=\frac{1}{2}$. We can use this idea to obtain the other two coefficients as well - the coefficient of $\omega(X,[X, Y])$ is 
the integral of the determinant

$$
\left|\begin{array}{cc}
1 & \frac{t}{2} \\
0 & \frac{s-s^{2}}{2}
\end{array}\right|=\frac{s-s^{2}}{2}
$$

which is $\frac{1}{12}$, and the coefficient of $\omega(Y,[X, Y])$ is the integral of the determinant

$$
\left|\begin{array}{cc}
t & \frac{t}{2} \\
s & \frac{s-s^{2}}{2}
\end{array}\right|=-\frac{s^{2} t}{2}
$$

which is $-\frac{1}{12}$.

As a final check on this calculation, let us again show that when $\omega$ is a cocycle, so is $\int \omega$. We know this must be true by Proposition 13, of course, but when checking it explicitly the cocycle condition seems almost miraculous. Since this final computation is a bit of a workout, we tuck it into the proof of the following proposition. It is only a check, and understanding the calculation is not necessary in light of Proposition 13.

Proposition 30. Let $N$ be a simply-connected Lie group whose Lie algebra $\mathfrak{n}$ is 2-step nilpotent. If $\omega$ is a Lie algebra 2-cocycle on $\mathfrak{n}$, then the Lie group 2-cochain on $N$ defined by

$$
\int \omega(g, h)=\frac{1}{2} \omega(X, Y)+\frac{1}{12} \omega(X-Y,[X, Y]),
$$

where $g=\exp (X)$ and $h=\exp (Y)$, is also a cocycle.

Proof. As already noted, this fact is immediate from Proposition 13, but we want to ignore this and check it explicitly. To do this, we repeatedly use the Baker-Campbell-Hausdorff formula, the assumption that $\mathfrak{n}$ is 2-step nilpotent, and the cocycle condition on $\omega$. This latter condition reads:

$$
d \omega(X, Y, Z)=-\omega([X, Y], Z)+\omega([X, Z], Y)-\omega([Y, Z], X)=0 .
$$

Note how this resembles the Jacobi identity. We prefer to write it as follows:

$$
\omega(X,[Y, Z])=\omega([X, Y], Z)+\omega(Y,[X, Z]) .
$$

To begin, the coboundary of the inhomogeneous Lie group 3-cochain $\int \omega$ is given by:

$$
d \int \omega(g, h, k)=\int \omega(h, k)-\int \omega(g h, k)+\int \omega(g, h k)-\int \omega(g, h) .
$$


Let us assume that

$$
g=\exp (X), \quad h=\exp (Y), \quad k=\exp (Z)
$$

so that

$$
g h=\exp \left(X+Y+\frac{1}{2}[X, Y]\right), \quad h k=\exp \left(Y+Z+\frac{1}{2}[Y, Z]\right) .
$$

Now we repeatedly insert the expression for our Lie group 2-cochain, so the coboundary of $\int \omega$ becomes:

$$
\begin{aligned}
d \int \omega(g, h, k)= & \frac{1}{2} \omega(Y, Z)+\frac{1}{12} \omega(Y-Z,[Y, Z])-\frac{1}{2} \omega\left(X+Y+\frac{1}{2}[X, Y], Z\right) \\
& -\frac{1}{12} \omega\left(X+Y+\frac{1}{2}[X, Y]-Z,[X+Y, Z]\right) \\
& +\frac{1}{2} \omega\left(X, Y+Z+\frac{1}{2}[Y, Z]\right) \\
& +\frac{1}{12} \omega\left(X-Y-Z-\frac{1}{2}[Y, Z],[X, Y+Z]\right) \\
& -\frac{1}{2} \omega(X, Y)-\frac{1}{12} \omega(X-Y,[X, Y])
\end{aligned}
$$

Note that the cocycle condition combined with nilpotency implies that any term in which $\omega$ eats two brackets vanishes. In general,

$$
\omega([X, Y],[Z, W])=\omega([[X, Y], Z], W)+\omega(Z,[[X, Y], W])=0,
$$

thanks to the fact that brackets of brackets vanish. So, in the expression for $d \int \omega$, we can simplify the fourth term:

$$
\begin{aligned}
\omega & \left(X+Y+\frac{1}{2}[X, Y]-Z,[X+Y, Z]\right) \\
& =\omega(X+Y-Z,[X+Y, Z])+\frac{1}{2} \omega([X, Y],[X+Y, Z]) \\
& =\omega(X+Y-Z,[X+Y, Z]) .
\end{aligned}
$$

Similarly for the sixth term:

$$
\omega\left(X-Y-Z-\frac{1}{2}[Y, Z],[X, Y+Z]\right)=\omega(X-Y-Z,[X, Y+Z]) .
$$


This leaves us with:

$$
\begin{aligned}
d \int \omega(g, h, k) & \\
= & \frac{1}{2} \omega(Y, Z)+\frac{1}{12} \omega(Y-Z,[Y, Z])-\frac{1}{2} \omega\left(X+Y+\frac{1}{2}[X, Y], Z\right) \\
& -\frac{1}{12} \omega(X+Y-Z,[X+Y, Z])+\frac{1}{2} \omega\left(X, Y+Z+\frac{1}{2}[Y, Z]\right) \\
& +\frac{1}{12} \omega(X-Y-Z,[X, Y+Z])-\frac{1}{2} \omega(X, Y)-\frac{1}{12} \omega(X-Y,[X, Y]),
\end{aligned}
$$

Expanding this using bilinearity, we obtain, after many cancellations:

$$
\begin{aligned}
d \int \omega(g, h, k)= & -\frac{1}{4} \omega([X, Y], Z)-\frac{1}{12} \omega(X,[Y, Z])-\frac{1}{12} \omega(Y,[X, Z]) \\
& +\frac{1}{4} \omega(X,[Y, Z])-\frac{1}{12} \omega(Y,[X, Z])-\frac{1}{12} \omega(Z,[X, Y]) .
\end{aligned}
$$

We combine the two terms with coefficient $1 / 4$ using the cocycle condition:

$$
-\omega([X, Y], Z)+\omega(X,[Y, Z])=\omega([Y, X], Z)+\omega(X,[Y, Z])=\omega(Y,[X, Z]) .
$$

Similarly, for the first and fourth terms with coefficient $1 / 12$, we apply the cocycle condition to get:

$$
\omega(X,[Y, Z])+\omega(Z,[X, Y])=\omega(Y,[X, Z]) .
$$

So, substituting these in, we finally obtain:

$$
\begin{aligned}
d \int \omega(g, h, k)= & \frac{1}{4} \omega(Y,[X, Z])-\frac{1}{12} \omega(Y,[X, Z])-\frac{1}{12} \omega(Y,[X, Z]) \\
& -\frac{1}{12} \omega(Y,[X, Z])=0,
\end{aligned}
$$

as desired.

As a corollary, note that we could equally well have said:

Corollary 31. Let $N$ be a simply-connected Lie group whose Lie algebra $\mathfrak{n}$ is 2-step nilpotent. If $\omega$ is a Lie algebra 2-cocycle on $\mathfrak{n}$, then the Lie group 2-cochain on $N$ defined by

$$
\int \omega(g, h)=\int_{0}^{1} \int_{0}^{1} \omega\left(X+t Y+\frac{t}{2}[X, Y], s Y+\frac{s-s^{2}}{2}[X, Y]\right) d s d t
$$

where $g=\exp (X)$ and $h=\exp (Y)$, is also a cocycle. 
Proof. By our calculation in this section,

$$
\int \omega(g, h)=\frac{1}{2} \omega(X, Y)+\frac{1}{12} \omega(X-Y,[X, Y]),
$$

so the result is immediate.

\section{3-cochains}

Let $\omega$ be a 3-cochain on the Lie algebra: that is, a left-invariant 3-form. Judging by our experience in the last section, the complexity of integrating $\omega$ to a Lie group 3-cochain may be quite high. Indeed, we shall ultimately avoid writing down $\int \omega$, except as an integral. Nonetheless, we can make this integral quite explicit.

We define the Lie group 3-cochain $\int \omega$ to be the integral of $\omega$ over a 3 -simplex in $N$. In particular:

$$
\int \omega(g, h, k)=\int_{[1, g, g h, g h k]} \omega .
$$

Now assume that $g=\exp (X), h=\exp (Y)$ and $k=\exp (Z)$. Recall that we obtain the 3 -simplex $[1, g, g h, g h k]$ using the apex-base construction: we connect each point of the base $[g, g h, g h k]=g[1, h, h k]$ to 1 by the exponential map. In the last section, we saw that $[1, h, h k](t, u)=\exp (t(Y+u Z+$ $\left.\left.\frac{u}{2}[Y, Z]\right)\right)$, so the base is parameterized by

$$
\begin{aligned}
{[g, g h, g h k](t, u) } & =g \exp \left(t\left(Y+u Z+\frac{u}{2}[Y, Z]\right)\right) \\
& =\exp \left(X+t Y+t u Z+\frac{t u}{2}[Y, Z]+\frac{1}{2}[X, t Y+t u Z]\right),
\end{aligned}
$$

by the Baker-Campbell-Hausdorff formula. Now let us construct $[1, g, g h$, $g h k]$ by first constructing a map from the cube

$$
\varphi:[0,1] \times[0,1] \times[0,1] \rightarrow N
$$

given by

$$
\begin{aligned}
\varphi(s, t, u) & =\exp \left(s\left(X+t Y+t u Z+\frac{t u}{2}[Y, Z]+\frac{1}{2}[X, t Y+t u Z]\right)\right) \\
& =\exp \left(s X+s t Y+s t u Z+\frac{s t}{2}[X, Y]+\frac{s t u}{2}[Y, Z]+\frac{s t u}{2}[X, Z]\right) .
\end{aligned}
$$


At this stage in our general construction, since this map is 1 on the $\{0\} \times$ $[0,1] \times[0,1]$ face of the cube and on the lines $\{s\} \times\{0\} \times[0,1]$ of constant $s$ on the $[0,1] \times\{0\} \times[0,1]$ face of the cube, we could quotient the cube out by these sets to obtain a map from the standard 3 -simplex. But in practice, we do not need to do this. Since the integral $\int_{[1, g, g h, g h k]} \omega$ is invariant under reparameterization, we might as well parameterize our 3-simplex $[1, g, g h, g h k]$ with $\varphi$ and integrate over the cube to obtain:

$$
\int \omega(g, h, k)=\int_{0}^{1} \int_{0}^{1} \int_{0}^{1} \omega\left(\frac{\partial \varphi}{\partial s}, \frac{\partial \varphi}{\partial t}, \frac{\partial \varphi}{\partial u}\right) d s d t d u
$$

Once again, our task has essentially reduced to computing the partial derivatives of $\varphi$, and once again, thanks to the left invariance of $\varphi$, we may as well left translate these partials back to 1 once we have them, since:

$$
\omega\left(\frac{\partial \varphi}{\partial s}, \frac{\partial \varphi}{\partial t}, \frac{\partial \varphi}{\partial u}\right)=\omega\left(\varphi^{-1} \frac{\partial \varphi}{\partial s}, \varphi^{-1} \frac{\partial \varphi}{\partial t}, \varphi^{-1} \frac{\partial \varphi}{\partial u}\right)
$$

Let us begin with $\frac{\partial \varphi}{\partial s}$. Since the exponent of $\varphi(s, t, u)$ is linear in $s$, this is simply:

$$
\frac{\partial \varphi}{\varphi s}(s, t, u)=\varphi(s, t, u)\left(X+t Y+t u Z+\frac{t}{2}[X, Y]+\frac{t u}{2}[Y, Z]+\frac{t u}{2}[X, Z]\right) .
$$

This is a tangent vector at $\varphi(s, t, u)$. We can left translate it back to 1 to obtain:

$$
\varphi^{-1} \frac{\partial \varphi}{\varphi s}=X+t Y+t u Z+\frac{t}{2}[X, Y]+\frac{t u}{2}[Y, Z]+\frac{t u}{2}[X, Z] .
$$

The partial with respect to $t$ is slightly harder, because the exponent is not linear in $t$. To compute this, we again need the Zassenhaus formula, Formula 3, to separate the terms linear in $t$ from those that are not. Applying this, we obtain

$$
\begin{aligned}
\varphi(s, t, u)= & \exp (s X) \exp \left(s t Y+s t u Z+\frac{s t}{2}[X, Y]+\frac{s t u}{2}[Y, Z]\right. \\
& \left.+\frac{s t u}{2}[X, Z]-\frac{1}{2}[s X, s t Y+s t u Z]\right) .
\end{aligned}
$$


Differentiating this with respect to $t$ and left translating the result to 1 , we get:

$$
\varphi^{-1} \frac{\partial \varphi}{\partial t}=s Y+s u Z+\frac{s}{2}[X, Y]+\frac{s u}{2}[Y, Z]+\frac{s u}{2}[X, Z]-\frac{1}{2}[s X, s Y+s u Z],
$$

which we can simplify by combining like terms:

$$
\varphi^{-1} \frac{\partial \varphi}{\partial t}=s Y+s u Z+\frac{s-s^{2}}{2}[X, Y]+\frac{s u}{2}[Y, Z]+\frac{s u-s^{2} u}{2}[X, Z] .
$$

Finally, the partial with respect to $u$ requires that we separate out the terms linear in $u$, again using the Zassenhaus formula:

$$
\begin{aligned}
\varphi(s, t, u)= & \exp \left(s X+s t Y+\frac{s t}{2}[X, Y]\right) \\
& \times \exp \left(s t u Z+\frac{s t u}{2}[Y, Z]+\frac{s t u}{2}[X, Z]-\frac{1}{2}[s X+s t Y, s t u Z]\right) .
\end{aligned}
$$

Differentiating this with respect to $u$ and left translating the result to 1 , we get:

$$
\varphi^{-1} \frac{\partial \varphi}{\partial u}=s t Z+\frac{s t}{2}[Y, Z]+\frac{s t}{2}[X, Z]-\frac{1}{2}[s X+s t Y, s t Z],
$$

which we can again simplify by combining like terms:

$$
\varphi^{-1} \frac{\partial \varphi}{\partial u}=s t Z+\frac{s t-s^{2} t^{2}}{2}[Y, Z]+\frac{s t-s^{2} t}{2}[X, Z] .
$$

Substituting these partial derivatives into the integral, our problem becomes:

$$
\begin{aligned}
\int \omega(g, h, k)= & \int_{0}^{1} \int_{0}^{1} \int_{0}^{1} \omega\left(X+t Y+t u Z+\frac{t}{2}[X, Y]+\frac{t u}{2}[Y, Z]\right. \\
& +\frac{t u}{2}[X, Z], s Y+s u Z+\frac{s-s^{2}}{2}[X, Y]+\frac{s u}{2}[Y, Z] \\
& +\frac{s u-s^{2} u}{2}[X, Z], s t Z+\frac{s t-s^{2} t^{2}}{2}[Y, Z] \\
& \left.+\frac{s t-s^{2} t}{2}[X, Z]\right) d s d t d u .
\end{aligned}
$$

This integral is bad enough. Further evaluating this integral is quite a chore (the answer involves 17 nonzero terms!), so we stop here. We would only 
like to give a hint as to how the evaluation could be done. As in the last section, thanks to $\omega$ 's trilinearity and antisymmetry, the coefficients of the terms in $\int \omega(g, h, k)$ are integrals of various determinants. For instance, the coefficient of $\omega(X, Y, Z)$ is the integral of the $3 \times 3$ determinant

$$
\left|\begin{array}{ccc}
1 & t & t u \\
0 & s & s u \\
0 & 0 & s t
\end{array}\right|=s^{2} t
$$

which we obtain from reading off the coefficients of $X, Y$ and $Z$ in the integrand. So the coefficient of $\omega(X, Y, Z)$ in $\int \omega(g, h, k)$ is $\int_{0}^{1} \int_{0}^{1} \int_{0}^{1} s^{2} t d s$ $d t d u=\frac{1}{6}$. The other terms may be computed similarly.

Just as we shall not attempt to evaluate the integral for $\int \omega(g, h, k)$, we also do not attempt to demonstrate that it gives a Lie group cocycle when $\omega$ is a Lie algebra cocycle. After all, Proposition 13 does this for us, so we immediately obtain:

Proposition 32. Let $N$ be a simply connected Lie group whose Lie algebra $\mathfrak{n}$ is 2-step nilpotent. If $\omega$ is a Lie algebra 3-cocycle on $\mathfrak{n}$, then the Lie group 3-cochain on $N$ given by

$$
\begin{aligned}
\int \omega(g, h, k)= & \int_{0}^{1} \int_{0}^{1} \int_{0}^{1} \omega\left(X+t Y+t u Z+\frac{t}{2}[X, Y]+\frac{t u}{2}[Y, Z]\right. \\
& +\frac{t u}{2}[X, Z], s Y+s u Z+\frac{s-s^{2}}{2}[X, Y]+\frac{s u}{2}[Y, Z] \\
& +\frac{s u-s^{2} u}{2}[X, Z], s t Z+\frac{s t-s^{2} t^{2}}{2}[Y, Z] \\
& \left.+\frac{s t-s^{2} t}{2}[X, Z]\right) d s d t d u,
\end{aligned}
$$

where $g=\exp (X), h=\exp (Y)$ and $k=\exp (Z)$, is also a cocycle.

\section{References}

[1] J. Azcárraga and J. Izquierdo, Lie groups, Lie algebras, cohomology and some applications to physics, Cambridge University Press, Cambridge, 1995, pp. 230-236.

[2] J. Baez, The octonions, Bull. Amer. Math. Soc. 39 (2002), 145-205. Also available as arXiv:math/0105155.

[3] J. Baez and A. Crans, Higher-dimensional algebra VI: Lie 2-algebras, TAC 12 (2004), 492-528. Available at http://www.tac.mta.ca/tac/ 
volumes/12/15/12-15abs.html. Also available as arXiv:math.QA/ 0307263.

[4] J. Baez and J. Huerta, The algebra of grand unified theories, Bull. Amer. Math. Soc. 47 (2010), 483-552. Also available as arXiv:0904.1556.

[5] J. Baez and J. Huerta, Divsion algebras and supersymmetry I, in Superstrings, Geometry, Topology, and $C^{*}$-algebras, eds. R. Doran, G. Friedman, J. Rosenberg, Proc. Symp. Pure Math. 81, AMS, Providence, 2010, 65-80. Also available as arXiv:0909.0551.

[6] J. Baez and J. Huerta, Division algebras and supersymmetry II, Adv. Theoret. Math. Phys. 15 (2011), 1373-1410. Available as arXiv:1003. 3436.

[7] J. Baez and J. Huerta, An invitation to higher gauge theory, Gen. Relativ. Gravit. 43 (2011), 2335-2392. Available at http://www.springer link.com/content/a4622gr730x5671m/. Also available as arXiv:1003. 4485.

[8] J. Baez and A. Lauda, Higher-dimensional algebra V: 2-groups, TAC 12 (2004), 423-491. Available at http://www.tac.mta.ca/tac/ volumes/12/14/12-14abs.html. Also available as arXiv:math/0307200.

[9] J. Baez and U. Schreiber, Higher gauge theory, in Categories in Algebra, Geometry and Mathematical Physics, eds. A. Davydov et al, Contemp. Math. 431, AMS, Providence, 2007, 7-30. Also available as arXiv:math/0511710.

[10] J. Baez, D. Stevenson, A. Crans and U. Schreiber, From loop groups to 2-groups, Homology Homotopy Appl. 9 (2007), 101-135. Available as arXiv:math/0504123.

[11] L. Balduzzi, C. Carmeli and R. Fioresi, A comparison of the functors of points of supermanifolds. Available as arXiv:0902.1824.

[12] L. Bonora, M. Bregola, R. D'Auria, P. Fr K. Lechner, P. Pasti, I. Pesando, M. Raciti, F. Riva, M. Tonin and D. Zanon, Some remarks on the supersymmetrization of the Lorentz Chern-Simons form in $D=10 N=1$ supergravity theories, Phys. Lett. B 277, 306-312. Also available at http://ncatlab.org/nlab/files/BonoraSuperGS.pdf.

[13] F. Borceux, Handbook of categorical Algebra 1: basic category theory, Cambridge University Press, Cambridge, 1994.

[14] F. Brandt, Supersymmetry algebra cohomology I: Definition and general structure, J. Math. Phys. 51, 122302, 2010. Also available as arXiv:0911.2118.

Supersymmetry algebra cohomology II: primitive elements in 2 and 3 dimensions, J. Math. Phys. 51 112303, 2010. Also available as arXiv: 1004.2978. 
- Supersymmetry algebra cohomology III: primitive elements in four and five dimensions, J. Math. Phys. 52 (2011), 052301. Also available as arXiv:1005.2102.

[15] A. Carey, S. Johnson and M. Murray, Holonomy on D-branes, J. Geom. Phys. 52 (2) (2004), 186-216. Also available as arXiv:hep-th/ $0204199 \mathrm{v} 3$.

[16] E. Cheng and A. Lauda, Higher-dimensional categories: an illustrated guidebook. Available at http://www.cheng.staff.shef.ac.uk/ guidebook/index.html.

[17] C. Chevalley and S. Eilenberg, Cohomology theory of Lie groups and Lie algebras, Trans. Amer. Math. Soc. 63 (1948), 85-124.

[18] K.-W. Chung and A. Sudbery, Octonions and the Lorentz and conformal groups of ten-dimensional space-time Phys. Lett. B 198 (1987), 161-164.

[19] A.M. Cohen, B. Lisser, M.A.A. van Leeuwen, et al., LiE, A computer algebra package for Lie group computations, version 2.2.2, the computer algebra group, Centre for Mathematics and Computer Science in Amsterdam. Available at http://www-math.univ-poitiers.fr/ maavl/ $\mathrm{LiE} /$.

[20] P. Deligne et al., eds., Quantum fields and strings: a course for mathematicians, Volume 1, Amer. Math. Soc., Providence, Rhode Island, 1999.

[21] M.J. Duff, Supermembranes: the first fifteen weeks, Class. Quantum Grav. 5 (1988), 189-205. Also available at http://ccdb4fs.kek.jp/cgibin/img_index?8708425.

[22] C. Ehresmann, Categories structurees, Ann. Ec. Normale Sup. 80 (1963).

— Categories structurees III: Quintettes et applications covariantes, Cahiers Top. et DG V(1963).

- Introduction to the theory of structured categories, Technical Report University of Kansas at Lawrence (1966).

[23] J.M. Evans, Supersymmetric Yang-Mills theories and division algebras, Nucl. Phys. B 298 (1988), 92-108. Also available as http://ccdb4fs.kek.jp/cgi-bin/img_index?8801412.

[24] R. Foot and G.C. Joshi, On a certain supersymmetric identity and the division algebras, Mod. Phys. Lett. A 3 (1988), 999-1004.

[25] D.S. Freed and E. Witten, Anomalies in string theory with D-branes, Asian J. Math. 3 (1999), 819. Also available as arXiv:hep-th/ 9907189.

[26] K. Gawedzki and N. Reis, WZW branes and gerbes, Rev. Math. Phys. 14 (2002), 1281-1334. Also available as arXiv:hep-th/0205233. 
[27] K. Gawedzki, Topological actions in two-dimensional quantum field theories, in Nonperturbative Quantum Field Theory, eds. G. t'Hooft, A. Jaffe, G. Mack, P.K. Mitter and R. Stora, Plenum, New York, 1988, pp. 101-141.

[28] E. Getzler, Lie theory for nilpotent $L_{\infty}$-algebras, Ann. Math. 170 (2009), 271-301. Available as arXiv:math. AT/0404003.

[29] M. Green, J. Schwarz and E. Witten, Superstring Theory, Volume 1, Cambridge U. Press, Cambridge, 1987. Appendix 4.A: Super YangMills theories, pp. 244-247. Section 5.1.2: The supersymmetric string action, 253-255.

[30] M. Green and J. Schwarz, Covariant description of superstrings, Phys. Lett. B 136 (1984), 367-370.

[31] A. Henriques, Integrating $L_{\infty}$-algebras, Compos. Math. 144 (2008), 1017-1045. Available as arXiv:math/0603563.

[32] J. Houard, An integral formula for cocycles of Lie groups, Annales de l'I.H.P. 32 (1980), 221-247. Available at http://archive.numdam.org/ article/AIHPA_1980_32_3_221_0.pdf.

[33] J. Huerta, Division algebras, supersymmetry and higher gauge theory, PhD Thesis, Department of Mathematics, UC Riverside, 2011. Available as arXiv:1106.3385.

[34] A. Hurwitz, Über die Composition der quadratischen Formen von beliebig vielen Variabeln, Nachr. Ges. Wiss. Göttingen (1898), 309-316.

[35] T. Kugo and P. Townsend, Supersymmetry and the division algebras, Nucl. Phys. B 221 (1983), 357-380. Also available at http://ccdb4fs. kek.jp/cgi-bin/img_index?198301032.

[36] T. Leinster, Basic bicategories. Available as arXiv:math/9810017.

[37] T. Leinster, A survey of definitions of n-category, TAC 10, 1-70. Available at http://www.tac.mta.ca/tac/volumes/10/1/10-01abs.html. Also available as arXiv:math/0107188.

[38] D. Leites, Cohomologies of Lie superalgebras, Funct. Anal. Appl. 9, 340-341.

[39] M. Markl, S. Schnider and J. Stasheff, Operads in algebra, topology and physics, AMS, Providence, Rhode Island, 2002.

[40] C. Manogue and A. Sudbery, General solutions of the covariant superstring equations of motion, Phys. Rev. D 40 (1989), 4073-4077.

[41] M. Movshev, A. Schwarz and R. Xu, Homology of Lie algebra of supersymmetries. Available as arXiv:1011.4731.

[42] — - Homology of Lie algebra of supersymmetries and of super Poincare Lie algebra, Nucl. Phys. B (2012), 483-503. Also available as arXiv:1106.0335. 
[43] M. Penkava, $L_{\infty}$ algebras and their cohomology. Available as arXiv:q-alg/9512014.

[44] C. Sachse, A categorical formulation of superalgebra and supergeometry. Available as arXiv:0802.4067.

[45] H. Sati, U. Schreiber and J. Stasheff, $L_{\infty}$-algebras and applications to string- and Chern-Simons n-transport. "Quantum Field Theory: Competitive Models", eds. B. Fauser, J. Toltsdorf, E. Zeidler, Birkhäuser Verlag, Basel, 2009, pp. 303-424. Also available as arXiv:0801.3480.

[46] M. Schlessinger and J. Stasheff, The Lie algebra structure of tangent cohomology and deformation theory J. Pure Appl. Alg. 38 (1985), 313-322.

[47] C. Schommer-Pries, A central extension of smooth 2-groups and a finitedimensional string 2-group, Geometry \& Topology 15 (2011), 609-676. Also available as arXiv:0911.2483.

[48] J. Schray, The general classical solution of the superparticle, Class. Quant. Gravit. 13 (1996), 27-38. Also available as arXiv:hep-th/ 9407045.

[49] J. Schray and C. Manogue, Octonionic representations of Clifford algebras and triality, Found. Phys. 26 (1996), 17-70. Also available as arXiv:hep-th/9407179.

[50] U. Schreiber, Differential cohomology in a cohesive topos. Habilitation thesis. Available at http://nlab.mathforge.org/schreiber/show/ differential+cohomology+in+a+cohesive+topos.

[51] A.S. Schwarz, On the definition of superspace, Teor. Mat. Fiz. 60 (1984), 37-42 (in Russian). English translation in Theor. Math. Phys. 60, 657-660.

[52] A. Sudbery, Division algebras, (pseudo)orthogonal groups and spinors, J. Phys. A 17 (1984), 939-955.

[53] G. Tuynman, Supermanifolds and supergroups: basic theory, Kluwer Academic Publishers, Dordrecht, 2004.

[54] A.A. Voronov, Mappings of supermanifolds, Teoret. Mat. Fiz. 60 (1984), 43-48 (in Russian). English translation in Th. Math. Phys. 60, 660-664.

[55] W.T. van Est, Group cohomology and Lie algebra cohomology in Lie groups, I and II, Indag. Math. 15 (1953), 484-504.

- On the algebraic cohomology concepts in Lie groups, I and II, Proc. Kan. Ned. Akad. 58 (1955), 225-233, 287-294. 
